\title{
Primary Effects of X-ray and Photo-Absorption Induced Excitations in Biomolecules
}

\author{
Dissertation \\ zur Erlangung des mathematisch-naturwissenschaftlichen \\ Doktorgrades \\ "Doctor rerum naturalium" \\ der Georg-August-Universität Göttingen \\ im Promotionsprogramm IMPRS-pbcs \\ der Georg-August University School of Science (GAUSS)
}

\author{
vorgelegt von \\ Carl Friedrich Burmeister \\ aus Braunschweig
}

Göttingen, 2013 
- Betreuungsausschuss

Prof. Dr. Helmut Grubmüller (Betreuer)

Theoretical and Computational Biophysics ${ }^{1}$

Prof. Dr. Tim Salditt

Institute for X-Ray Physics ${ }^{2}$

PD Dr. Simone Techert

Structure Dynamics of (Bio)Chemical Systems ${ }^{1}$

- Prüfungskommission

Prof. Dr. Helmut Grubmüller (Referent)

Prof. Dr. Tim Salditt (Koreferent)

PD Dr. Simone Techert

Dr. Gerrit Groenhof

Nanoscience Center ${ }^{3}$

Prof. Dr. Sarah Köster

Institute for X-Ray Physics ${ }^{1}$

Prof. Dr. Marcus Müller

Institute for Theoretical Physics ${ }^{1}$

${ }^{1}$ Max Planck Institute for Biophysical Chemistry

${ }^{2}$ Georg-August University Göttingen

${ }^{3}$ University of Jyväskylä, Finland

Tag der mündlichen Prüfung: 11.04.2013 
Hiermit erkläre ich, dass ich die vorliegende Arbeit selbstständig verfasst und keine anderen als die angegebenen Quellen und Hilfsmittel verwendet habe.

Göttingen, den 28.02.2013

\section{Carl Burmeister}





\section{Contents}

$\begin{array}{ll}\text { Abstract } & \text { xxi }\end{array}$

Zusammenfassung xxiii

1 Introduction 1

I X-ray Absorption Induced Electron Dynamics 7

2 Theory 9

2.1 Hartree-Fock Theory . . . . . . . . . . . . . . . . . . . . . . 9 9

2.1.1 Time-dependent Hartree-Fock . . . . . . . . . . . . . 11

2.1.2 Linear Combination of Atomic Orbitals . . . . . . . . . . . . . 12

3 Model System 17

3.1 Implementation . . . . . . . . . . . . . . . . . 18





3.3 .1 Four-particle System . . . . . . . . . . . . . . 23

3.3 .2 Six-particle System . . . . . . . . . . . . . . . 24

4 Beryllium $\quad 27$

4.1 Numerical Hartree-Fock . . . . . . . . . . . . . . . . . . . . . . . 29

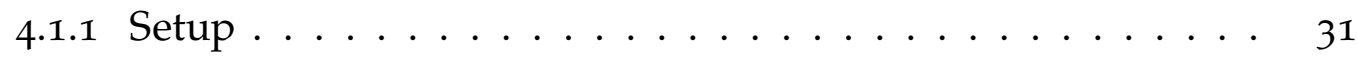

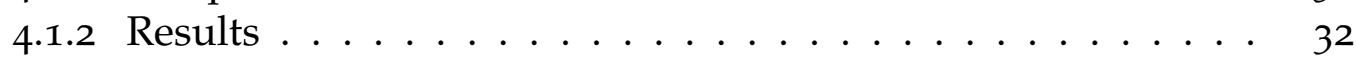

4.2 B-spline Hartree-Fock . . . . . . . . . . . . . . . . . . . . . 35

4.2 .1 Setup ........................... 37

4.2 .2 Results ........................... 39 
5 Molecules $\quad 41$

5.1 Implementation . . . . . . . . . . . . . . . . 42

5.2 Charge Migration Analysis . . . . . . . . . . . . . . . . . 42

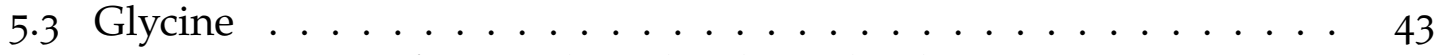

5.3.1 Ionization from 11th Molecular Orbital . . . . . . . . . 43

$5 \cdot 3.2$ Ionization from 16th Molecular Orbital . . . . . . . . . 46

5.3.3 Comparison with Literature Results . . . . . . . . . . . . 47

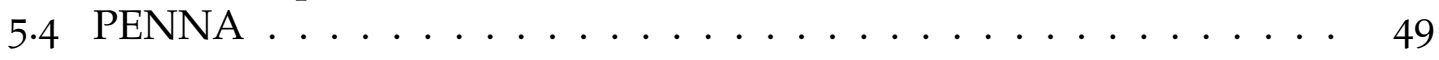

6 Conclusion \& Outlook 53

II Photo-Absorption Induced Molecular Dynamics 57

7 Conical Intersections 59

7.1 Potential Energy Surfaces . . . . . . . . . . . . . . . . . 59

7.2 Surface Intersections $\ldots \ldots \ldots$. . . . . . . . . . . 61

8 Methods $\quad 63$

8.1 Molecular Dynamics . . . . . . . . . . . . . . . . . . 65

8.1.1 Force Fields . . . . . . . . . . . . . . . . . . . 65

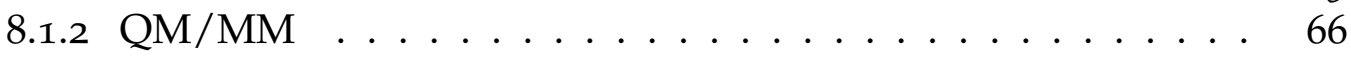

8.2 Excited State Quantum Chemistry . . . . . . . . . . . . . . 66

8.3 Diabatic Surface Hopping . . . . . . . . . . . . . . . . . . . . 69

8.4 Fewest Switches Surface Hopping . . . . . . . . . . . . 70

9 Applications 73

9.1 Protonated Schiff Base . . . . . . . . . . . . . . . . . 74

9.1.1 Excited State Dynamics . . . . . . . . . . . . . . . 75

9.1.2 Comparison of Hopping Algorithms . . . . . . . . . 76

9.2 Photoactive Yellow Protein . . . . . . . . . . . . . . 80

9.2.1 Setup ...................... 82

9.2.2 Excited State Dynamics . . . . . . . . . . . . . . . . . 82

9.2.3 Summarized Results . . . . . . . . . . . . . . . . . . 84

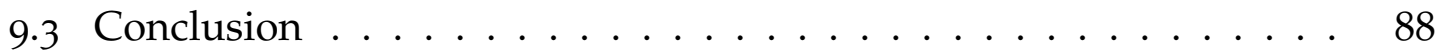

10 Excitation Energy Transfer 91

10.1 Setup . . . . . . . . . . . . . . . . . . . . . . . . . . . . . . . . . . . . . . 94




10.2.1 Ab Initio Results . . . . . . . . . . . . . . . . . . . 95

10.2.2 DSH Trajectory . . . . . . . . . . . . . . . . . . . . . . . . 98

10.2.3 FSSH Trajectories . . . . . . . . . . . . . . . . . . . . . . . 98

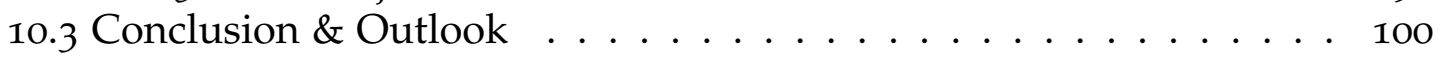

A Molecular structures 105

A.1 Glycine . . . . . . . . . . . . . . . . . . 105

A.2 PENNA ........................... 106

A.3 Ab Initio Data of $\mathrm{P}_{44} \ldots \ldots \ldots$. . . . . . . . . . . . . . . . . . . .

$\begin{array}{ll}\text { Bibliography } & 109\end{array}$ 



\section{List of Abbreviations}

\begin{tabular}{|c|c|}
\hline $\begin{array}{l}\mathrm{ADC} \\
\mathrm{AIMD} \\
\mathrm{AO}\end{array}$ & $\begin{array}{l}\text { algebraic diagrammtic construction } 41,47,48 \\
\text { ab initio molecular dynamics } 63,64 \\
\text { atomic orbital } 13-15,28\end{array}$ \\
\hline CAS & complete active space $73,82,83,85-88,102$ \\
\hline CASSCF & $\begin{array}{l}\text { complete active space self-consistent field } 64,67 \text {, } \\
69,71,74,82,94\end{array}$ \\
\hline $\mathrm{CC}$ & coupled cluster 55,63 \\
\hline $\mathrm{CI}$ & configuration interaction $48,54,55,64,66-69$ \\
\hline CIS & configuration interaction singles 54,55 \\
\hline CIS(D) & $\begin{array}{l}\text { configuration interaction singles with perturba- } \\
\text { tion treatment of doubles } 55\end{array}$ \\
\hline CISD & configuration interaction singles and doubles 55 \\
\hline CMA & charge migration analysis 42,43 \\
\hline $\mathrm{CMF}$ & constant mean field $14,30,42$ \\
\hline Cys & Cysteine 80,82 \\
\hline DC & decoherence correction $71,72,74,77-79,85-88$ \\
\hline DFT & density functional theory 63 \\
\hline DFVP & Dirac-Frenkel variational principle 11 \\
\hline DOF & degrees of freedom 10,63 \\
\hline DSH & $\begin{array}{l}\text { diabatic surface hopping } 64,69,70,73-75,77-79 \text {, } \\
82,85-89,95,97,98\end{array}$ \\
\hline EET & excitation energy transfer 5, 6, 91-93, 97 \\
\hline EMSL & Environmental Molecular Sciences Laboratory 43, \\
\hline EOM-CC & $\begin{array}{l}49 \\
\text { equation-of-motion coupled cluster } 67\end{array}$ \\
\hline FCI & full configuration interaction 69 \\
\hline $\mathrm{FF}$ & force field $63,65,66,82,94$ \\
\hline FRET & Foerster resonance energy transfer $5,6,91$ \\
\hline FSSH & fewest switches surface hopping $60,64,71-74,77-$ \\
\hline
\end{tabular}


HF Hartree-Fock 3, 9-13, 18, 19, 28, 31-33, 35, 39, 40, $42,55,64,68$

$\mathrm{HOMO}$ highest occupied molecular orbital 41, 45, 49, 50

LCAO linear combination of atomic orbitals 12, 13

LUMO lowest unoccupied molecular orbital 48

MCSCF multi-configurational self-consistent field 64, 67, 68

MD molecular dynamics 4-6, 59, 61, 63-65, 69, 73, 74, $81,88,89,91-94,102$

MEP minimal energy pathway 94, 95, 97, 98, 100

MM molecular mechanics 66

MO molecular orbital 10-13, 19, 20, 22-26, 28, 29, 31$33,35,39-50,53-55,64,68,69,74,82$

OPDM one-electron density matrix 13, 19, 20

PCA p-coumaric acid 80

PDB protein data base 80

PENNA 2-phenylethyl-N,N-dimethylamine 41, 49-51, 54

PES potential energy surface $4-6,59-65,69,73,86,88$, 91-93

PSB protonated Schiff base 73

PSB2 protonated Schiff base with two double bonds 5 , $73,74,79,88$

PYP photoactive yellow protein 5, 73, 80, 88, 94

QM quantum mechanics 66, 71, 82

QM/MM quantum mechanics/molecular mechanics 64, 88, 94, 100, 102

RHF restricted Hartree-Fock 13, 14, 19, 22, 32, 38

ROG radius of gyration $23-25,32,39$

SA state averaging 82,94

SCF self-consistent field 13, 14, 18, 19, 30 
TDDFT time-dependent density functional theory 4,41 , 67

TDHF time-dependent Hartree-Fock 4, 12, 14, 17-19, 26$28,30,34,35,41-43,47-49,51,53-55$ 



\section{List of Figures}

1.1 Single molecule scattering. . . . . . . . . . . . . 2

1.2 Elastic scattering and photo-ionization with subsequent Auger decay. 3

$3.1 \quad$ Model potential for $Z=6 \ldots \ldots \ldots \ldots \ldots \ldots$

3.2 Time-evolution of the diagonal elements of $\boldsymbol{D}^{\prime}$ corresponding to the doubly occupied molecular orbitals in the six-particle ground state. 20

$3.3 D^{\alpha^{\prime}}(t)$ as function of time for various grid sizes. . . . . . . 21

3.4 Transition in the six-particle system after instantaneous core-shell ionization for different timesteps. . . . . . . . . . . . . 22

3.5 Occupation of the initial molecular orbitals in the four particle system. 23

3.6 Radius of gyration of the occupied molecular orbitals in the four particle system. . . . . . . . . . . . . . . . . 24

3.7 Occupation of the initial molecular orbitals in the six-particle system. 25

3.8 Radius of gyration of the occupied molecular orbitals in the sixparticle system. . . . . . . . . . . . . . . . . 26

4.1 Spectrum of the ground state Fock matrix with the numerical grid. $3^{1}$

4.2 Radius of gyration of the occupied molecular orbitals during the numerical grid simulation. . . . . . . . . . . . . 32

4.3 Occupation of the initial molecular orbitals during the numerical grid simulation. . . . . . . . . . . . . . . 33

4.4 Eigenvalue spectra of the ground state Fock matrix using the Bspline basis. . . . . . . . . . . . . . . . . 37

4.5 Radius of gyration of the occupied molecular orbitals during the B-spline simulations. . . . . . . . . . . . . . . 39

4.6 Occupation of the initial molecular orbitals during the B-spline sim-

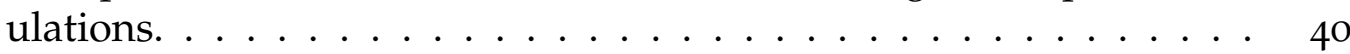

5.1 Glycine structure and the ionized molecular orbitals. . . . . . . . 44 
5.2 Hole occupation after ionization of the 11th molecular orbital in

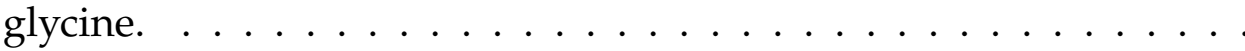

5.3 Hole occupation after ionization of the 16th molecular orbital in

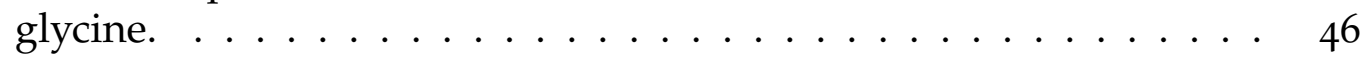

5.4 Sketch of the PENNA structure $\ldots \ldots \ldots \ldots \ldots \ldots$

5.5 Time-evolution after ionization from the highest occupied molecular orbital in PENNA. . . . . . . . . . . . . . . 50

8.1 Sketch of the mechanical embedding scheme. . . . . . . . . . . 67

8.2 The complete active space decomposition of the molecular orbital

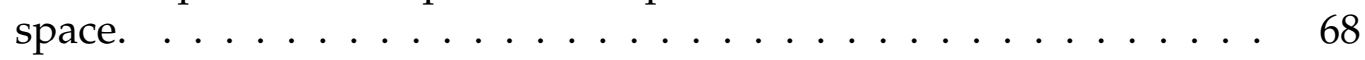

9.1 Active space used for the PSB2 calculations. . . . . . . . . . . 74

9.2 Example excited state dynamics trajectory of PSB2. . . . . . . . 75

9.3 Excited state lifetimes of PSB2. . . . . . . . . . . . 76

9.4 Distribution of bond lengths (left) and dihedral angels (right) at the

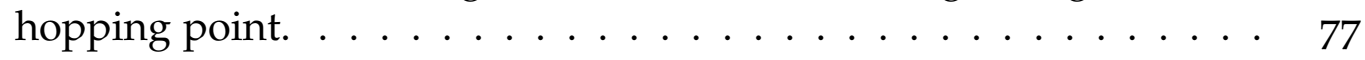

9.5 Photoactive yellow protein. . . . . . . . . . . . 80

9.6 The early phase of the photocycle of the photoactive yellow protein. 81

9.7 Definition of atom names in the PYP chromophore. . . . . . . . 81

9.8 Trans-to-cis isomerization of the double bond in PYP. . . . . . . . 83

9.9 Trans-to-trans isomerization of the single bond in PYP. . . . . . . 84

9.10 A "trapped" FSSH trajectory of PYP. . . . . . . . . . . . . . 85

10.1 "Down hill" excitation energy transfer. . . . . . . . . . . . . 92

10.2 Barrier crossing excitation energy transfer. . . . . . . . . . . . 92

10.3 Pnn structure. . . . . . . . . . . . . . . . 93

10.4 Sketch of the minimal energy pathway of the excitation energy transfer reaction of P44. . . . . . . . . . . . . . . 96

10.5 DSH trajectory switching diabatic state while evolving around a conical intersection. . . . . . . . . . . . . . . . 97

10.6 Potential energies of the three excited states of the diabatic surface hopping trajectory. . . . . . . . . . . . . . . . 99

10.7 Potential energies of the three excited states of the fewest switches trajectory started from the 1 ns snapshot . . . . . . . . . . . 99

10.8 Potential energies of the three excited states of the fewest switches trajectory started from the 6 ns snapshot. . . . . . . . . . . 101 
10.9 Potential energies of the three excited states of the fewest switches trajectory started from the 9 ns snapshot. . . . . . . . . . . . 101

A.1 $\operatorname{CASSCF}(14,12) / 6-31 G^{*}$ optimized $C_{2}$ structure of P44. $\quad \ldots \ldots . \quad 107$ 



\section{List of Tables}

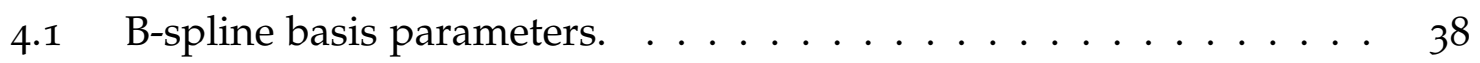

9.1 Expectation values and standard deviations of the excited state lifetime. . . . . . . . . . . . . . . . . . 77

9.2 The products of the photo-isomerization in PSB2. . . . . . . . 78

9.3 Bootstrapped results of the photo-isomerization in PSB2. . . . . 78

9.4 P-values isomerization results. . . . . . . . . . . . . . 79

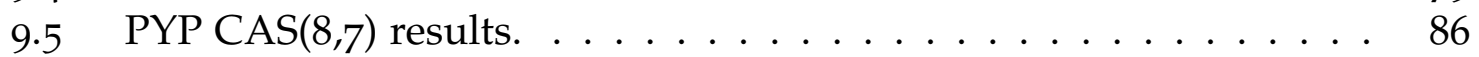

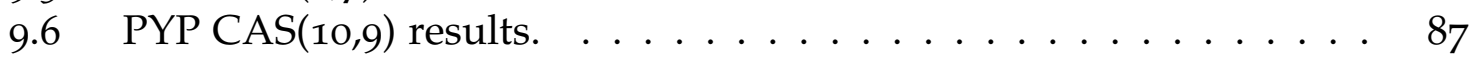

10.1 Minimal energy conical intersections between the electronic states

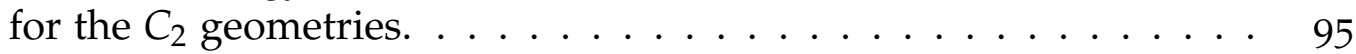

10.2 Conical intersections encountered in the diabatic surface hopping



A.1 Glycine equilibrium structure. . . . . . . . . . . . . 105

A.2 PENNA equilibrium structure. . . . . . . . . . . . 106

A.3 $\operatorname{CASSCF}(14,12) / 6-31 G^{*}$ optimized $C_{2}$ structures of P44. . . . . 107

A.4 $\operatorname{CASSCF}(14,12) / 6-31 G^{*}$ energies of P44 . . . . . . . . . 108 



\section{List of Algorithms}

2.1 Simple iterative algorithm to solve the Roothan-Hall equations . . . 14

2.2 Pseudo code of the constant mean field algorithm[1] . . . . . . . 15 



\section{Abstract}

To see whether single molecule scattering experiments can yield atomic resolution structures of biomolecules, it is necessary to understand the physics underlying the radiation damage processes induced by the $X$-ray radiation. The first part of this work is concerned with the electron dynamics during the Auger decay as part of the radiation damage processes in single molecule scattering experiments. To test if Hartree-Fock theory suffices to describe auto-ionization processes like the Auger decay, we simulated the electron dynamics in a one-dimensional model system and a beryllium atom after instantaneous core-shell ionization using time-dependent Hartree-Fock theory. The simulations employed both numerical grids as well as B-splines. In a model system containing six particles the initially created core-hole is refilled during the electron dynamics. But no clear emission of a particle into the continuum occurred. During the electron dynamics in a beryllium atom, however, neither a refilling of the initially created core-hole nor the emission of a particle was observed. As two different, flexible basis sets were used it is likely to be the limitation of Hartree-Fock theory causing the absence of the Auger process in the simulations. In addition, we used the approach described above to simulate the electron dynamics after instantaneous valence-shell ionization in two small organic molecules. The electron dynamics exhibit a diffusion-like behavior of the valence hole. These dynamics, however, do not reproduce the charge migration dynamics predicted by correlated ab-initio methods.

To compare different hopping algorithms and to validate previous simulation results, excited state molecular dynamics simulations of the photo-isomerization dynamics of a small retinal model and the photo-active yellow protein were performed in the second part of this thesis. In the retinal model small differences in the excited state lifetimes and quantum yields are observed among the different hopping algorithms. The simulations of the photo-active yellow protein chromophore yield a photo-isomerization pathway that agrees with the isomerization pathway previously predicted. There is, however, a large difference in the excited state lifetimes among the hopping algorithms. Additionally, the excited state molecular dynamics simulations are used to study the molecular dynamics induced excitation energy transfer in a small bi-chromophoric molecule. The molecular dynamics simulations together with ab-initio calculations indicate that molecular dynamics on different nonadiabatically coupled potential energy surfaces can explain the excitation energy transfer from one chromophore to another. This offers a different explanation of excitation energy transfer processes in small molecules compared to Förster and Dexter theory. 



\section{Zusammenfassung}

Um zu verstehen, ob Einzelmolekülstreuexperimente Stukturen von Biomolekülen mit atomarer Auflösung liefern können, ist ein Verständnis der Physik notwendig, die den durch die Röntgenstrahlung ausgelösten Strahlungsschäden zugrunde liegt. Im ersten Teil dieser Arbeit wird die Elektronendynamik während des Augerzerfalls untersucht. Der Augerzerfall ist ein wichtiger Teil des Strahlungsschadens in Einzelmolekülstreuexperimenten. Um zu testen, ob Hartree-Fock-Theorie ausreicht um Autoionisationsprozesse wie den Augerzerfall zu beschreiben, haben wir die Elektronendynamik nach plötzlicher Ionisation der Kernschale in einem eindimensionalen Modell und einem Berylliumatom simuliert. In den Simulationen wurden sowohl numerische Gitter als auch B-bpline-Basen verwendet. Im Modellsystem mit sechs Teilchen wird das Loch in der Kernschale während der Elektronendynamik aufgefüllt. Allerdings wird kein Teilchen in das Kontinuum emittiert. Während der Elektronendynamik im Berylliumatom wird weder das Loch in der Kernschale aufgefüllt noch wird ein Elektron in das Kontinuum emittiert. Da zwei verschiedene, flexible Basen verwendet wurden, ist wahrscheinlich die Hartree-Fock-Näherung selbst unzureichend, um den Augerprozess zu beschreiben. Zusätzlich wurde mithilfe des obigen Ansatzes die Elektronendynamik nach Valenzionisation in zwei kleinen, organischen Molekülen simuliert. Die beobachtete Elektronendynamik ähnelt einer Diffusion des Valenzloches. Dies steht im Gegensatz zu der sogenannten "charge migration" Dynamik, die mithilfe von korrelierten ab-initio Methoden vorhergesagt wurde.

Um verschiedene "surface hopping" Algorithmen miteinander zu vergleichen und Ergebnisse von vorangegangenen Simulationen zu validieren, wurde im zweiten Teil die Photoisomerisierungsdynamik eines kleinen Retinalmodells und des Chromophores des "photo-active yellow protein" mit Moleküldynamiksimulationen für angeregte Zustände und verschiedenen "surface hopping" Algorithmen simuliert. Im Fall des Retinalmodells sind kleine Unterschiede in den Lebensdauern des angeregten Zustandes und den Produkten der Isomerisierung zwischen den unterschiedlichen Algorithmen festzustellen. Der Photoisomerisierungsmechanismus, der in den Simulationen des "photo-active yellow protein" beobachtet wurde, stimmt mit dem Photoisomerisierungsmechanismus, der in vorherigen Studien beschrieben worden ist, überein. Allerdings gibt es deutliche Unterschiede in den von den verschiedenen Algorithmen vorhergesagten Lebensdauern des angeregten Zustandes. Zusätzlich wurden die Moleküldynamiksimulationen verwendet, um Anregungstransferprozesse in kleinen Molekülen mit zwei Chromophoren zu untersuchen. Zusammen mit ab-initio Berechnungen deuten die Moleküldynamiksi- 
mulationen darauf hin, dass der Anregungstransfer von einem Chromophor zum anderen durch Moleküldynamik auf verschiedenen, nicht adiabatisch gekoppelten Potentialflächen erklärt werden kann. Dieser Ansatz bietet also eine alternative Erklärung für Anregungstransferprozesse in kleinen Molekülen im Vergleich zur Förster- und Dextertheorie. 


\section{1 \\ Introduction}

Atomic resolution structures of proteins are crucial for the understanding of life processes at the molecular level. These structures are usually obtained by Xray crystallography[2]. The first protein structure was solved in 1958 when John Kendrew and co-workers were able to obtain the structure of a whale myoglobin[3]. Since then X-ray crystallography has become one of the standard tools in structural biology.

The crystallization of proteins is one of the most important bottle necks in obtaining new atomic resolution structures as there is no general way to crystallize a given protein $[4,5]$. The next generation X-ray sources - the free electron lasers currently deployed in Germany[6], Japan[7] and the USA[8] — will provide X-ray pulses with a pulse length of $10-100 \mathrm{fs}$ at a wavelength of about $1 \AA$ with $10^{12}$ photons per pulse. These pulse parameters offer the possibility of a new type of experiment.

In single molecule scattering (Figure 1.1), a stream of single particles is inserted into the beam line. Each of these particles is hit by a single X-ray laser pulse and the scattering image is recorded. As indicated in Figure 1.1 the target molecule is destroyed during the illumination. Hence, this approach is also called "diffractand-destroy" method[10].

The radiation damage it caused by photo-ionization (see Figure 1.2 (mid)), which occurs about ten times more often then elastic scattering (see Figure 1.2 (left)) for a wavelength of $1 \AA[11]$.

In crystallography the radiation damage problem is overcome by distributing the damage over a whole ensemble of molecules. The short pulses provided by the next generation $X$-ray source allow a different strategy. Rather then spreading the radiation damage over an ensemble of molecules the goal of single molecule scattering is to outrun the radiation damage by collecting the scattering image before the molecule is destroyed. For this strategy to be successful, the critical question is: 


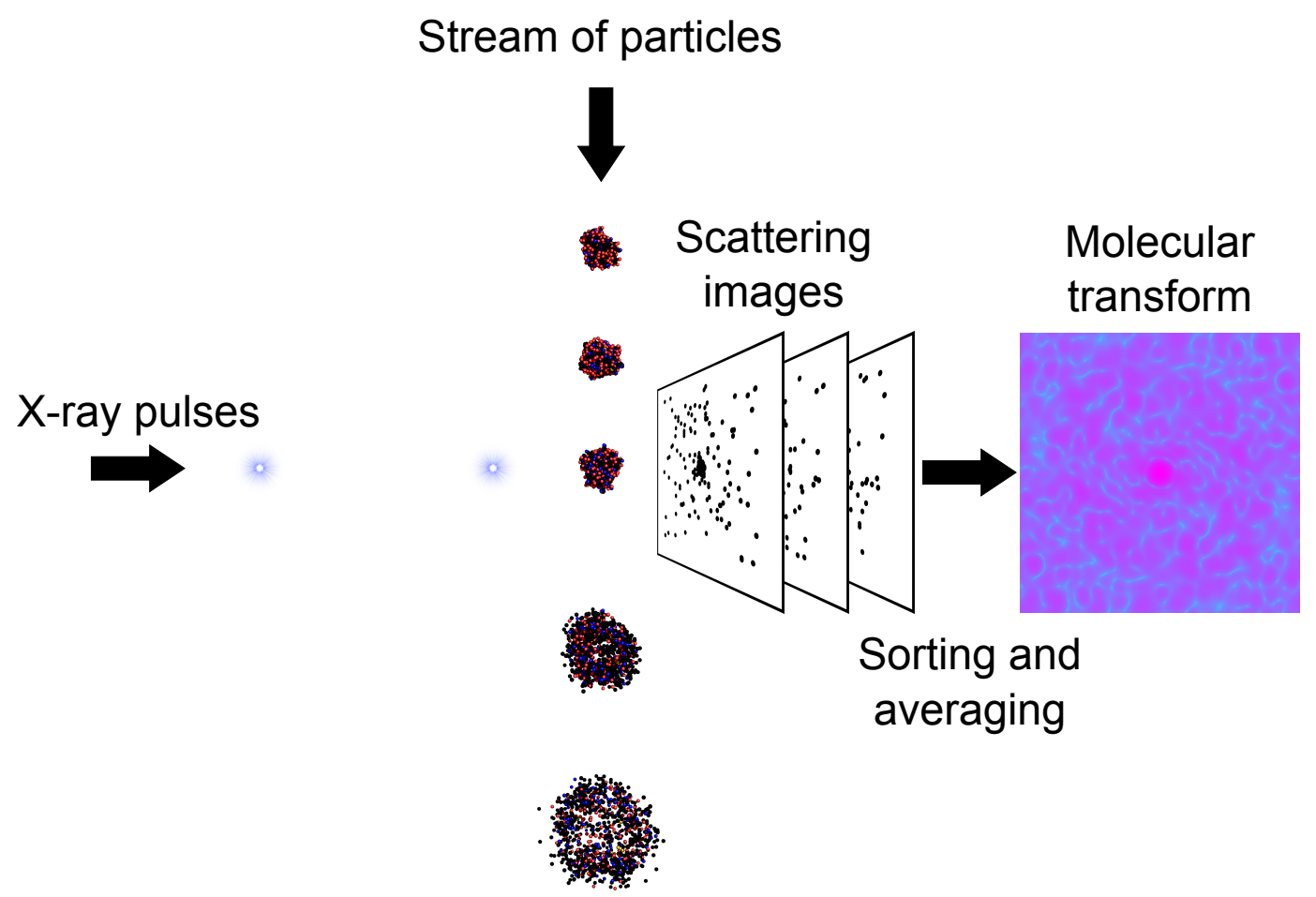

Figure 1.1: The single molecule scattering approach is sketched. A stream of single particles is inserted into the beam line. Every particle is illuminated with a single X-ray pulse and the scattering image is recorded. Afterwards, the recorded images are sorted and averaged accordingly to yield the structure of the molecule in reciprocal space[9].

How fast are the individual processes that lead to the radiation damage and how short do the X-ray pulses actually have to be to outrun these processes. Another related question is: Can these radiation damages be slowed down? It is, thus, necessary to understand the physics underlying the destruction process to estimate the pulse length needed to outrun the destruction process. The Auger decay (see Figure 1.2 (right)) plays an important role during the first part of the Coulomb explosion as it enhances the radiation damage caused by the photo-ionization. The Auger decay speeds up the Coulomb explosion in two ways. Firstly, an electron is emitted while the core vacancy is refilled charing up the molecule. Secondly, the refilled core shell can again be ionized by photo-ionization. For the "heavy" atoms (mostly carbon, nitrogen and oxygen found in proteins) the core-hole lifetime is of 

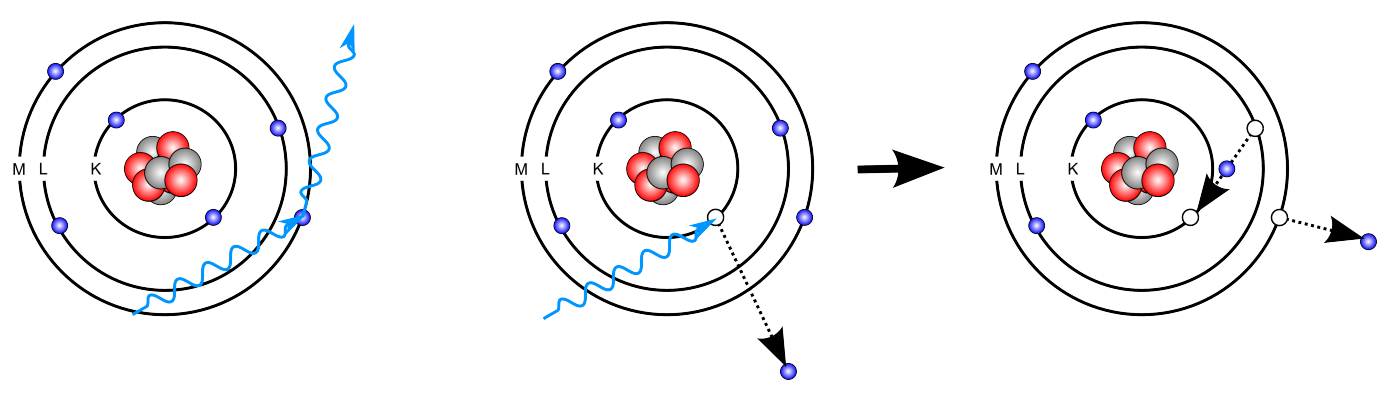

Figure 1.2: The left figure sketches elastic scattering. A photon (wavy blue line) is scattered from an electron (blue circle) changing its direction. The energy of the photon is conserved during elastic scattering leaving the state of the electron untouched. The right figure sketches photo-ionization with subsequent Auger decay. A photon is absorbed emitting a core electron. In the subsequent Auger decay the hole in the core shell is by a valence electron. In turn another valence electron is emitted and the energy is conserved.

the order of $5-10 \mathrm{fs}[12]$, which is about the same order of magnitude as the pulse length $10-100 \mathrm{fs}$.

The aim of Part I of this thesis is to study the electron dynamics during the Auger decay right after photo-ionization by solving the electronic time-dependent Schrödinger equation using computer simulations. Due to the high computational costs of quantum mechanical calculations this approach is not applicable to a whole protein. We will, thus, study the electron dynamics in smaller systems to understand how the environment of a specific atom influences the electron dynamics during the Auger decay after core shell ionization of the atom. The insight drawn from the electron dynamics simulation could later be used to improve existing statistical models [13-15] to simulate the Coulomb explosion of a whole protein. It is still preferable to use a computationally comparably cheap method. Hartree-Fock (HF) theory (cf. Section 2.1) is the computationally simplest ab initio method to solve the electronic Schrödinger equation.

It has been shown that highly correlated methods can be used to simulate autoionizing processes after inner valence ionization[16]. In contrast, stationary HF theory neglects any correlation effects. One could, thus, question whether the HF approximation is sufficient to describe the Auger decay. Note, however, that time-dependent HF theory will recover parts of the dynamic correlation effects, since a change of a part of the electron density will induce changes in the rest of 
the electron density. Hence, it is crucial to validate that time-dependent HartreeFock (TDHF) ${ }^{1}$ theory[18] is sufficient to describe the electron dynamics during the Auger decay process.

First, the applicability of TDHF to auto-ionization processes was tested using a one-dimensional model system (cf. Chapter 3), which imitates a single atom. In contrast to an atom in a one-dimensional system, it was not necessary to split the system into an angular and a radial part. This simplified both the derivation and implementation of the working equations as well as the interpretation of the simulations. A one-dimensional model simulation thus offered a natural starting point for this work.

To avoid possible limitations of $L^{2}$ basis sets, a numerical grid was used as a basis[19]. A grid offers the flexibility to describe both bound and continuum electrons, which occur during the Auger process. The method developed to solve the TDHF equations for the model system was then applied to atoms (cf. Chapter 4). As atoms are spherically symmetric they can be treated as being essentially onedimensional, only considering the radial part. Additionally to the numerical grid basis from the model system also a B-spline basis[20-22] was used. The B-spline offers a more balanced description of the bound and the continuum part of the system than the grid. This leads to more compact basis sets and allows longer simulations.

Unfortunately, the simple basis sets used for single atoms cannot be applied to molecules as they are lacking the spherical symmetry. To test the TDHF simulations on molecules, they were applied to the electron dynamics in small organic molecules after valence-shell photo-ionization (Chapter 5). In particular the electron dynamics obtained by TDHF were compared to the charge migration process $[23,24]$ predicted by correlated ab initio methods.

In Part II the dynamics induced by photo instead of X-ray absorption were considered. The absorption of an (optical) photon promotes the electronic wavefunction of a molecule into an excited state. The change of the electronic state changes the potential energy surface (PES) (cf. Chapter 7 ) for the nuclear wavefunction and, hence, induces molecular dynamics (MD).

An important example of an MD process induced by photo-absorption is photoisomerization. In photo-isomerization the sudden change of the PES induces a rotation of a dihedral angle and the excited electronic state decays radiationless through a conical intersection (cf. Section 7.2)[25]. The change in the molecular

\footnotetext{
${ }^{1}$ Note that that here TDHF does not refer to the calculation of excited states by linear response theory which is also called TDHF or time-dependent density functional theory (TDDFT)[17].
} 
structure server as the first step in many different biological processes leading to biological functions like vision or ion-pumping[26, 27].

To study these photo-isomerization processes by computer simulations in their native environment, e.g. proteins in solution[26], one has to reside on the classical approximation for the nuclei because wavepacket dynamics are only applicable to systems containing few atoms[28].

To describe the MD during a photo-isomerization properly, accurate forces exerted by the different electronic wavefunctions on the nuclei are required. To this end $a b$ initio quantum chemical methods were employed to determine the electronic wavefunction and the respective forces on the nuclei.

During the radiationless decay the molecule changes from one PES to another. This was facilitated in the MD simulations by so called hopping algorithms [29] (cf. Sections 8.3 and 8.4) that determine when exactly the hop to a different PES takes place.

One aim of this part is to compare different hopping algorithms to be able to judge the results obtained with them. Thereto, the excited state dynamics of the retinal model system protonated Schiff base with two double bonds (PSB2) were studied in vacuum using different hopping algorithms.

The photo-isomerization of the photoactive yellow protein (PYP) chromophore found in Halorhodospira halophila bacteria is thought to provide the first step in a cascade leading to photo-avoidance of the bacteria protecting it from harmful ultra violet light[30, 31].

The photo-isomerization of the PYP is experimentally[31-35] and computationally characterized[36-39]. To validate previous excited state MD simulation, predicting the trans-to-cis photo-isomerization dynamics in the PYP [36], two different hopping algorithms were used together with a better quantum chemically description of the excited states of the chromophore to simulate the photo-isomerization dynamics of the PYP.

Another important process induced by photo-absorption is excitation energy transfer (EET). In EET one chromophore, the donor, is excited by absorbing a photon. Subsequently, the excitation is transferred to a second chromophore, the acceptor, which excited state decays via photo-emission. Thus, an absorption in the absorption spectrum of the donor induces an emission in the emission spectrum of the acceptor. The EET is usually interpreted by Foerster resonance energy transfer (FRET)[40] or Dexter's[41] theory. In both theories the EET is attributed to perturbative coupling between the (distinct) wavefunctions of the two chromophores and is discussed in terms of Fermi's golden rule. 
Excitation energy transfer forms for instance the basis for the experimental technique also called FRET, which is used to determine inter- and intra-molecular distances on the nanometer scale and is widely used in biophysics [42, 43].

It has been, however, predicted that excited state MD on different nonadiabatically coupled PES can induce EET[44-47]. In small bi-chromophotic molecules this process offers an additional explanation for EET compared to the conventional interpretation of EET by FRET or Dexter's theory.

To test this prediction, we have performed excited state MD simulations mentioned above to study the excited state MD simulation for a bi-chromophoric pcyclophane. Speiser and co-workers[48] have shown experimentally that EET occurs in theses molecules depending on their size. 
Part I

\section{X-ray Absorption Induced Electron Dynamics}





\section{2 \\ Theory}

Here, the theoretical framework used for the subsequent calculations of the electron dynamics will be derived. The solution of the time-dependent electronic Schrödinger equation (atomic units, i.e. $e=m_{e}=\hbar=\frac{1}{4 \pi \varepsilon_{0}}=1$, are used throughout this work if not stated otherwise)

$$
i \frac{\partial}{\partial t} \Psi_{\text {elec }}=\hat{H}_{\text {elec }} \Psi_{\text {elec }}
$$

will be approximated using HF theory. The electronic Hamiltonian is given by

$$
\hat{H}_{\text {elec }}=\frac{1}{2} \sum_{j}^{n_{\text {elec }}} \Delta_{j}+\sum_{k>j}^{n_{\text {elec }}} \frac{1}{\left|\boldsymbol{r}_{j}-\boldsymbol{r}_{k}\right|}+\sum_{j A}^{n_{\text {elec }} N_{\text {Atoms }}} \frac{Z_{A}}{\left|\boldsymbol{r}_{j}-\boldsymbol{R}_{A}\right|},
$$

where $j, k, \boldsymbol{r}_{j}$ and $\boldsymbol{r}_{k}$ refer to electrons and their positions; $A, \boldsymbol{R}_{A}$ and $Z_{A}$ to nuclei and their positions and charges. In the following the subscripts elec and Atoms are dropped if the context is obvious.

\subsection{Hartree-Fock Theory}

In HF theory the wavefunction $\Psi$ is approximated by an anti-symmetrized product ansatz of single particle wavefunctions, usually called a Slater determinant. The electrons only interact with each other via mean-field potential[49]. Due to the single determinant ansatz and the mean-field interaction, electron correlation is not described by stationary HF theory[50]. In the HF theory the electronic wavefunc- 
tion $\Psi$ is approximated by a single Slater determinant formed by single particle functions

$$
\Psi(\boldsymbol{x} ; \boldsymbol{R}) \approx \hat{\mathcal{A}} \prod_{j}^{n} \chi_{j}\left(\boldsymbol{x}_{j} ; \boldsymbol{R}\right)=\left|\begin{array}{cccc}
\chi_{1}\left(\boldsymbol{x}_{1} ; \boldsymbol{R}\right) & \chi_{2}\left(\boldsymbol{x}_{1} ; \boldsymbol{R}\right) & \cdots & \chi_{n}\left(\boldsymbol{x}_{1} ; \boldsymbol{R}\right) \\
\chi_{1}\left(\boldsymbol{x}_{2} ; \boldsymbol{R}\right) & \chi_{2}\left(\boldsymbol{x}_{2} ; \boldsymbol{R}\right) & \cdots & \chi_{n}\left(\boldsymbol{x}_{2} ; \boldsymbol{R}\right) \\
\vdots & & & \\
\chi_{1}\left(\boldsymbol{x}_{n} ; \boldsymbol{R}\right) & \chi_{2}\left(\boldsymbol{x}_{n} ; \boldsymbol{R}\right) & \cdots & \chi_{n}\left(\boldsymbol{x}_{n} ; \boldsymbol{R}\right)
\end{array}\right|,
$$

with the combined space-spin coordinates $x=(\boldsymbol{r}, \sigma)$. The single particle functions $\chi_{1}\left(x_{1} ; R\right)$ are called molecular orbital (MO). The MOs are orthonormal[49]

$$
\left\langle\chi_{j} \mid \chi_{k}\right\rangle=\int \mathrm{d} x \chi_{j}^{*}(\boldsymbol{x}) \chi_{k}(\boldsymbol{x})=\delta_{j k} .
$$

The bra-ket notation indicates integration over the electronic spin-space degrees of freedom (DOF). Both the total wavefunction and the MOs depend parametrically on the nuclear positions $\boldsymbol{R}$ due to the nuclear potential term in the electronic Hamiltonian (2.2). The HF energy is given by[49]

$$
\begin{aligned}
E & =\langle\Psi|\hat{H}| \Psi\rangle=\sum_{j}^{n}\left\langle\chi_{j}\left|-\frac{1}{2} \Delta_{j}-\sum_{A}^{N} \frac{Z_{A}}{\left|\boldsymbol{r}_{j}-\boldsymbol{R}_{A}\right|}\right| \chi_{j}\right\rangle \\
& +\underbrace{\sum_{k>j}^{n}\left\langle\chi_{j}\left(\boldsymbol{x}_{1}\right) \chi_{k}\left(\boldsymbol{x}_{2}\right)\left|\frac{1}{\left|\boldsymbol{r}_{j}-\boldsymbol{r}_{k}\right|}\right| \chi_{j}\left(\boldsymbol{x}_{1}\right) \chi_{k}\left(\boldsymbol{x}_{2}\right)\right\rangle}_{\text {Coulomb }} \\
& -\underbrace{\sum_{k>j}^{n}\left\langle\chi_{j}\left(\boldsymbol{x}_{1}\right) \chi_{k}\left(\boldsymbol{x}_{2}\right)\left|\frac{1}{\left|\boldsymbol{r}_{j}-\boldsymbol{r}_{k}\right|}\right| \chi_{k}\left(\boldsymbol{x}_{1}\right) \chi_{j}\left(\boldsymbol{x}_{2}\right)\right\rangle}_{\text {Exchange }} \\
& =\sum_{j}^{n}\langle j(\hat{h} \mid j)+\frac{1}{2} \sum_{j k}^{n} \underbrace{(j j \mid j k)}_{\text {Coulomb }}-\underbrace{(j k \mid k j)}_{\text {Exchange }},
\end{aligned}
$$

with the one-electron operator $\hat{h}$ and the two-electron integrals $(p q \mid r s)$ in chemists notation[49]. Note that the Coulomb interaction acts among all MOs, whereas the exchange interaction only affects MOs of the same spin. The MOs are determined such that the HF energy is minimized, retaining the orthonormality of the MOs 
(2.4). To ensure the orthonormality of the MOs, the method of undetermined multipliers is used[51]. The HF equations are obtained from the first order variations of the Lagrange functions[49]

$$
\mathcal{L}=E-\sum_{j k}^{n} \varepsilon_{j k}\left(\langle j \mid k\rangle-\delta_{j k}\right) .
$$

This yields

$$
\hat{f} \chi_{j}=\left(\hat{h}+\sum_{k} \hat{J}_{k}-\hat{K}_{k}\right) \chi_{j}=\sum_{k} \varepsilon_{j k} \chi_{k},
$$

which has to be fulfilled for all $j$ for the first order variation of $\mathcal{L}$ to vanish. In (2.7) the Fock operator $\hat{f}=\hat{h}+\sum_{k} \hat{J}_{k}-\hat{K}_{k}$ is defined. Equations (2.7) are the $\mathrm{HF}$ equations in the noncanonical form. The Equations (2.7) define the MOs $\chi_{j}$ only up to a unitary transformation. This unitary transformation can be used to diagonalize the matrix of Lagrange multipliers $\varepsilon$ and to bring the HF equations into their canonical form

$$
\hat{f} \chi_{j}=\varepsilon_{j} \chi_{j}
$$

The Lagrange multipliers $\varepsilon_{j}$ can now be interpreted as orbital energies. Note that the HF equations are nonlinear as the Fock operator $\hat{f}$ depends on the orbitals due to the Coulomb and Exchange interactions.

\subsubsection{Time-dependent Hartree-Fock}

Time-dependent HF theory is derived from the time-dependent Schrödinger Equation (2.1) using the Dirac-Frenkel variational principle (DFVP)[18]. In the DFVP the action

$$
S=\int_{t_{0}}^{t_{1}} \mathrm{~d} t\left\langle\Psi\left|\hat{H}-i \frac{\partial}{\partial t}\right| \Psi\right\rangle
$$


is minimized with respect to variations of the wavefunction $\Psi$. Again, the wavefuntion $\Psi$ approximated by a Slater determinant

$$
\Psi(t, x ; \boldsymbol{R}) \approx \hat{\mathcal{A}} \prod_{j}^{n} \chi_{j}\left(t, x_{j} ; \boldsymbol{R}\right),
$$

where the MOs $\chi_{j}\left(t, x_{j} ; \boldsymbol{R}\right)$ are now time-dependent. The variations of the orbitals $\delta \chi_{j}$ are restricted as follows

$$
\langle\delta j \mid k\rangle=0 \forall j, k
$$

This restriction is physically meaningful as changes in the space of occupied MOs only introduce a phase factor that has no physical effect. The first order variation of the action $\delta S$ can then be expressed as[52,53]

$$
\delta S=\left\langle\delta \Psi\left|\hat{H}-i \frac{\partial}{\partial t}\right| \Psi\right\rangle
$$

which yields the TDHF equations

$$
i \frac{\partial}{\partial t} \chi_{j}=\hat{f} \chi_{j}
$$

\subsubsection{Linear Combination of Atomic Orbitals}

To solve the stationary (2.8) and time-dependent (2.13) Equations numerically, the linear combination of atomic orbitals (LCAO) approach is used. Accordingly, the MOs are expanded using a set of spacial basis functions $\{\mu(\boldsymbol{r})\}_{\mu=1 \ldots N}$

$$
\chi_{j}(\boldsymbol{x})=\chi_{j}^{\sigma}=\sum_{\mu}^{N} C_{\mu j}^{\sigma} \mu(\boldsymbol{r}) .
$$

The basis functions $\{\mu(\boldsymbol{r})\}_{\mu=1 \ldots N}$ are taken to be real. Inserting Equation (2.14) into the stationary HF Equations (2.8), projecting onto a basis function and integrating over space yields the Roothan-Hall equations

$$
\boldsymbol{F}^{\sigma} \boldsymbol{C}^{\sigma}=\varepsilon^{\sigma} \boldsymbol{S} \boldsymbol{C}^{\sigma}
$$


The Fock matrix $\boldsymbol{F}^{\sigma}$ is given by

$$
\begin{aligned}
F_{\mu \nu}^{\sigma} & =(\mu|\hat{f}| v)=\int \mathrm{d} \boldsymbol{r} \mu(\boldsymbol{r}) \hat{f} v(\boldsymbol{r}) \\
& =h_{\mu \nu}+\sum_{\lambda \rho}^{N}\left(D_{\lambda \rho}^{\alpha}+D_{\lambda \rho}^{\beta}\right)(\mu \nu \mid \rho \lambda)-D_{\lambda \rho}^{\sigma}(\mu \lambda \mid \rho v),
\end{aligned}
$$

with the one- and two-electron integrals in atomic orbitals (AOs) space

$$
\begin{aligned}
h_{\mu \nu} & =(\mu|\hat{h}| \nu), \\
(\mu \nu \mid \rho \lambda) & =\int \mathrm{d} \boldsymbol{r}_{1} \mathrm{~d} \boldsymbol{r}_{2} \mu\left(\boldsymbol{r}_{1}\right) \nu\left(\boldsymbol{r}_{1}\right) \frac{1}{\left|\boldsymbol{r}_{1}-\boldsymbol{r}_{2}\right|} \rho\left(\boldsymbol{r}_{2}\right) \lambda\left(\boldsymbol{r}_{2}\right),
\end{aligned}
$$

and the one-electron density matrixs (OPDMs)

$$
D_{\mu \nu}^{\sigma}=\sum_{j}^{n_{\sigma}} C_{\mu j} C_{v j}^{*}
$$

The $S$ matrix is the overlap matrix of the basis set

$$
S_{\mu v}=(\mu \mid v) .
$$

As the Fock operator (2.7) depends on the $\mathrm{MO}$, the Fock matrix $\boldsymbol{F}$ depends on the coefficient matrices $C^{\sigma}$. Thus, the Roothan-Hall Equations (2.15) are nonlinear and need to be solved iteratively. A solution of the Roothan-Hall equation is usually called self-consistent field (SCF) as the MOs are eigenfunctions of the corresponding Fock operator. In listing 2.1 a simple iterative algorithm is sketched to solve the Roothan-Hall equations. This algorithm will be later on used to determine the closed shell ground states of the model systems (cf. 3) and the atom (cf. 4). For a closed shell system the restricted Hartree-Fock (RHF) ansatz is used, where the same coefficients are used for both spins, i.e. $C^{\alpha}=C^{\beta}$. That means, all spatial are doubly occupied by electrons of opposing spin.

Similarly, inserting the LCAO ansatz (2.14) into the time-dependent HF Equations (2.13) yields

$$
i S \dot{C}^{\sigma}=\boldsymbol{F}^{\sigma} C^{\sigma} .
$$






Again these equations are nonlinear because the Fock matrices $\boldsymbol{F}^{\sigma}$ depend on the coefficient matrices $C^{\sigma}$. For a both stable and efficient time-propagation of the Equations (2.20) a modification of the constant mean field (CMF) algorithm[1] is used. Listing 2.2 sketches the modified CMF algorithm. The time-evolution operator $e^{-i \frac{\Delta t}{2} \boldsymbol{F}^{\sigma}}$ is evaluated by diagonalizing the Fock matrix $\boldsymbol{F}^{\sigma}$. This is always possible as $\boldsymbol{F}^{\sigma}$ is hermitian. The diagonilization of $\boldsymbol{F}^{\sigma}$ is again done in a space where $S=\mathbf{1}$ as in algorithm 2.1. This transformation step is, however, left out for clarity.

The TDHF theory will be used to study the electron dynamics in a one-dimensional model system (cf. Chapter 3), in atoms (cf. Chapter 4) and small molecules (cf. 5). The initial conditions for all simulations are set up using a RHF wavefunction. For the model system and the atoms the RHF wavefunction is converged using the SCF algorithm 2.1. The initial state is then propagated in time using the CMF algorithm sketched in 2.1 for both of the atom and the molecules. 


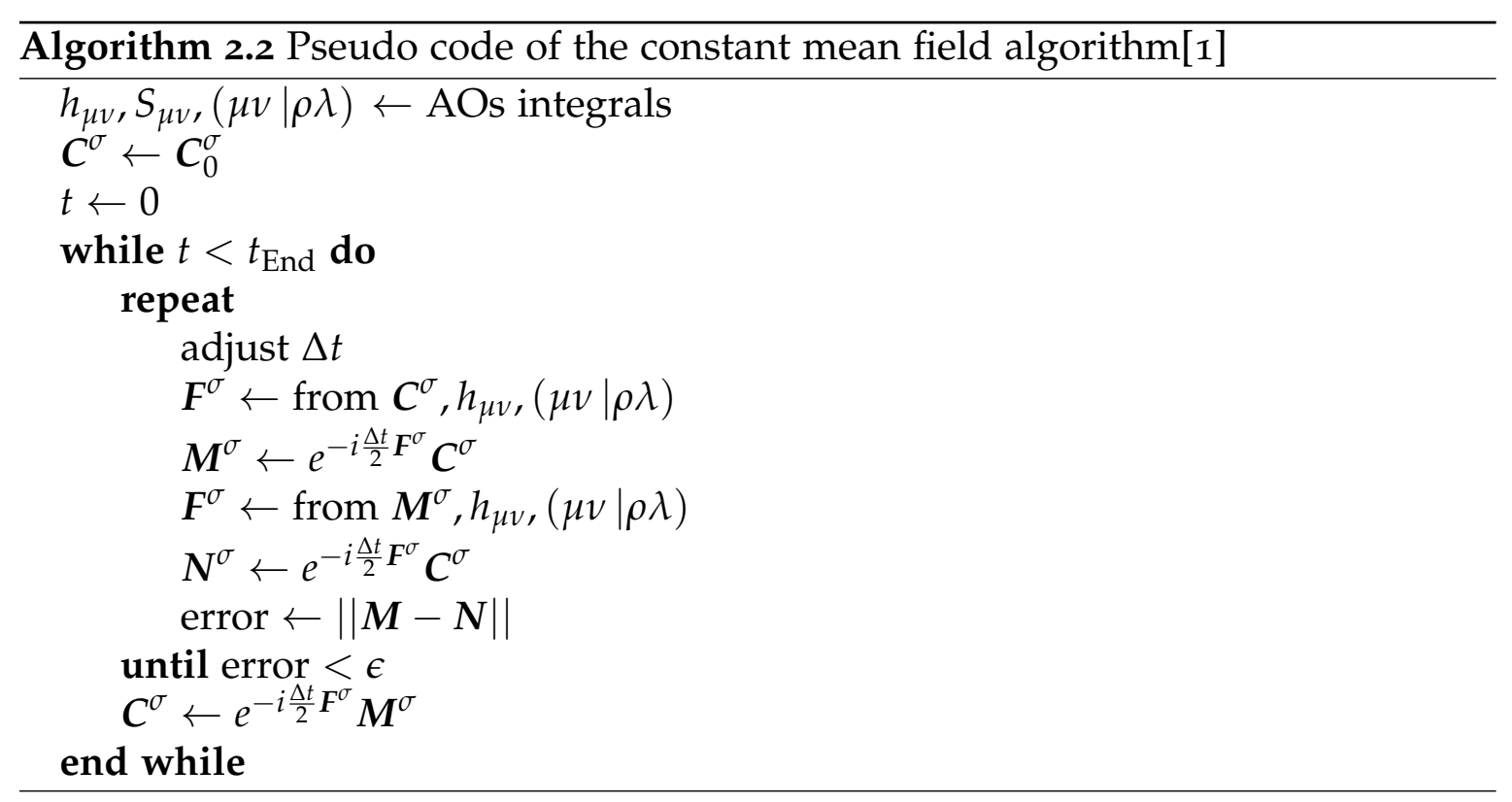





\section{3 \\ Model System}

The aim of this chapter is to test if time-dependent Hartree-Fock (TDHF) theory suffices to describe auto-ionizing processes despite the limited description of correlation by TDHF theory. To this end, the electron dynamics in a one-dimensional model system are studied using the methods discussed in the previous chapter. A screened Coulomb potential (see Figure 3.1) is used for both the central potential

$$
V^{\text {cental }}(r)=-\frac{Z}{\sqrt{r^{2}+1}}
$$

and the particle-particle interaction

$$
V^{\mathrm{pp}}\left(r_{12}\right)=\frac{1}{\sqrt{r_{12}^{2}+1}}
$$

because in one dimension the integrals over the Coulomb potential do not converge. This model has similar physical properties as an atom. It has a set of bound orbitals (see Figure 3.1) as well as continuum orbitals because the central potential (3.1) has an upper bound. Additionally, the restrictions of the TDHF theory with respect to correlation effects are the same in the model as in atoms because the correlation arises from the anti-symmetry of the wavefunction. Thus, we expect to see similar electron dynamics in the model as in atoms.

For an Auger-like process that is a transition during which the initial hole in the core-shell is refilled by a valence particle with the same spin. At the same time a valence particle with opposite spin is emitted into the continuum. Such a transition in the simulation would be a hint that TDHF is able to describe the Auger process. 




Figure 3.1: Sketch of the screened Coulomb potential for $Z=6$ and the corresponding ground state Hartree-Fock orbitals for the six particle ground state.

\subsection{Implementation}

For a proper description of both the bound and continuum orbitals a numerical grid is used instead of an $L^{2}$ basis set. In contrast to usual grid based HartreeFock (HF) implementations[19, 54], the grid is used as a basis to directly expand the orbitals. For a good description of the orbitals the grid spacing has to be significantly smaller then the smallest feature of the orbitals.

In the grid approximation the one- and two-electrons potential integrals are given by

$$
\begin{aligned}
\left(\mu\left|V^{\text {central }}\right| v\right) & =-\delta_{\mu \nu} \frac{Z}{\left|r_{\mu}\right|}, \\
(\mu \nu \mid \rho \lambda) & =\delta_{\mu \nu} \delta_{\rho \lambda} \frac{1}{\left|r_{\mu}-r_{\rho}\right|} .
\end{aligned}
$$

For the kinetic energy operator the second order central difference approximation is used

$$
\left(\mu\left|-\frac{1}{2} \Delta\right| \nu\right)=\delta_{\mu \nu}-\frac{1}{2}\left(\delta_{\mu(v-1)}+\delta_{\mu(v+1)}\right) .
$$

For the determination of the HF ground state wavefunction the simple selfconsistent field (SCF) procedure sketched in Listing 2.1 is used. The TDHF equa- 
tions are propagated in time using the fourth-order Runge-Kutta algorithm[55] which turned out the be sufficiently stable (see next section).

For the determination of the HF ground state the SCF algorithm sketched in Algorithm 2.1 is used.

\subsection{Setup}

The initial state of the simulation is supposed to approximate the electronic wavefunction right after a photo-ionization. The ionization was not described explicitly but the effect of the ionization was modeled by an instantaneous removal of a particle, i.e. a particle was removed from the wavefunction without relaxing it. This sudden approximation is valid as long as the energy of the photo electron is large enough[56]. The photon energy produced by the X-ray free-electron lasers is about $12 \mathrm{keV}$ while the binding energies of the core-shell electrons of carbon, nitrogen and oxygen are of the order of $300 \mathrm{eV}-500 \mathrm{eV}$ [57]. Hence, the kinetic energy of the photo electron exceeds the binding energy of any of the remaining electrons by about one and a half orders of magnitude. Thus, the initial state should be well described within the sudden approximation.

According to the discussion above, the initial state was prepared by converging a restricted Hartree-Fock (RHF) ground state wavefunction using the SCF algorithm discussed in Section 2.1.2. Then a particle was removed from the core orbital.

Before the actual simulation can be performed it is necessary to test the stability of the integrator used to propagate the TDHF equations in time and to determine a proper grid size and timestep for the time-propagation. The simulations of the sixparticle system provide as benchmarks as the dynamics of the six-particle system show a transition we are mainly interested in (for detailed discussion of the results consider Section 3.3.2). The transition was determined from the time-dependent one-electron density matrix $(\mathrm{OPDM}) \boldsymbol{D}_{00}^{\alpha}(t)$ projected into the space of the initial molecular orbitals (MOs)

$$
\boldsymbol{D}^{\alpha \prime}(t)=\boldsymbol{C}^{\dagger}(0) \boldsymbol{D}^{\alpha}(t) \boldsymbol{C}(0) .
$$

Similarly to the Mulliken population analysis[58] the diagonal elements of $D^{\alpha^{\prime}}(t)$ give the occupation of the initial orbitals.

First, the stability of the fourth-order Runge-Kutta integrator was tested. For this purpose, the converged RHF ground state of the six-particle system was propagated in time. The ground state was obtained using the SCF algorithm sketched 
in 2.1 with a convergence criterion of $\Delta E<10^{-7}$. As the converged ground state is an eigenstate no dynamics are expected beyond an oscillating phase factor for each orbital. This phase factor drops out when the time-dependent OPDM is considered.

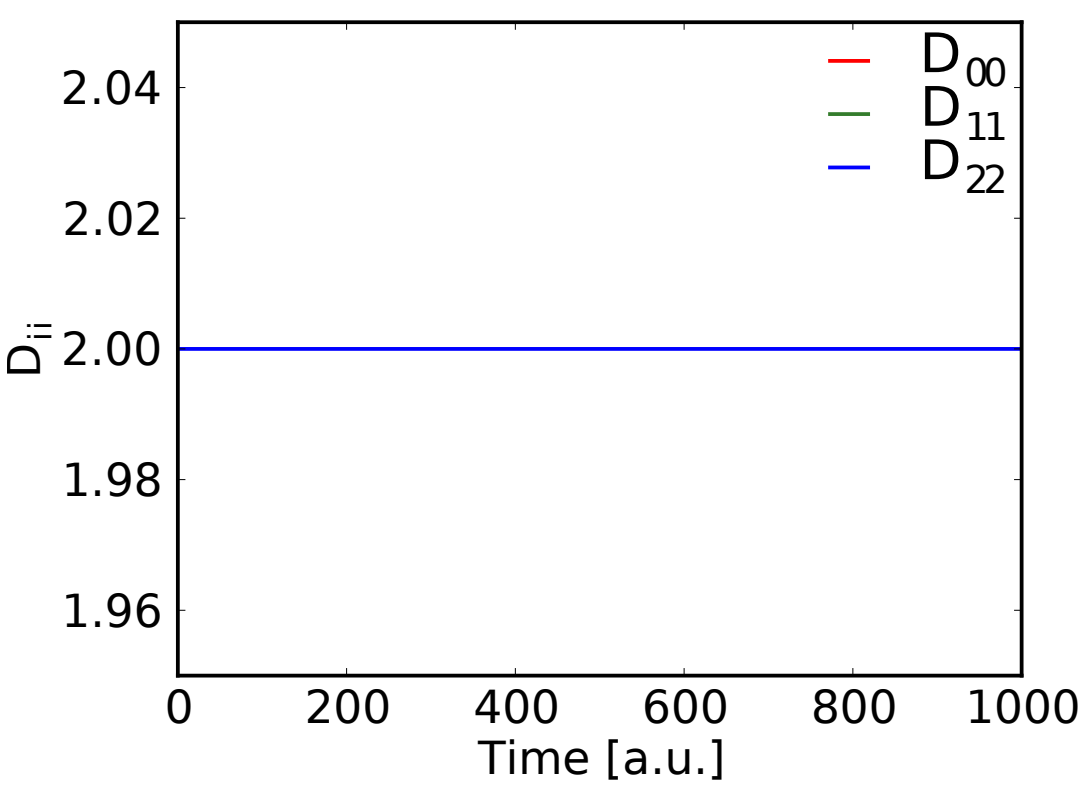

Figure 3.2: Time-evolution of the diagonal elements of $D^{\prime}$ corresponding to the doubly occupied molecular orbitals in the six-particle ground state. Note that all three curves overlay.

Figure 3.2 shows the time-evolution of the diagonal elements of $D^{\prime}$ corresponding to the doubly occupied orbitals of the six-particle ground state. As expected there is no change in the occupation of the MO allowing the conclusion that the ground state is properly converged and the fourth-order Runge-Kutta integrator is stable at least within the 1000 a.u. considered here.

Second, an appropriate grid size was determined. For that purpose, dynamics simulations of the six-particle system were performed using different grid sizes after removal of a particle from the core-shell $\chi_{0}^{\alpha}$ orbital. All the simulations were performed using a timestep of 0.001 a.u..

Figure 3.3 shows the respective $D^{\alpha \prime}{ }_{00}(t)$ elements of the OPDM as a function of time. For the first $200 \mathrm{a} . \mathrm{u}$. to $600 \mathrm{a} . \mathrm{u}$., depending on the grid size used, there is no change in the occupation of the initial $\chi_{0}^{\alpha}$ orbital. After this initial delay the 


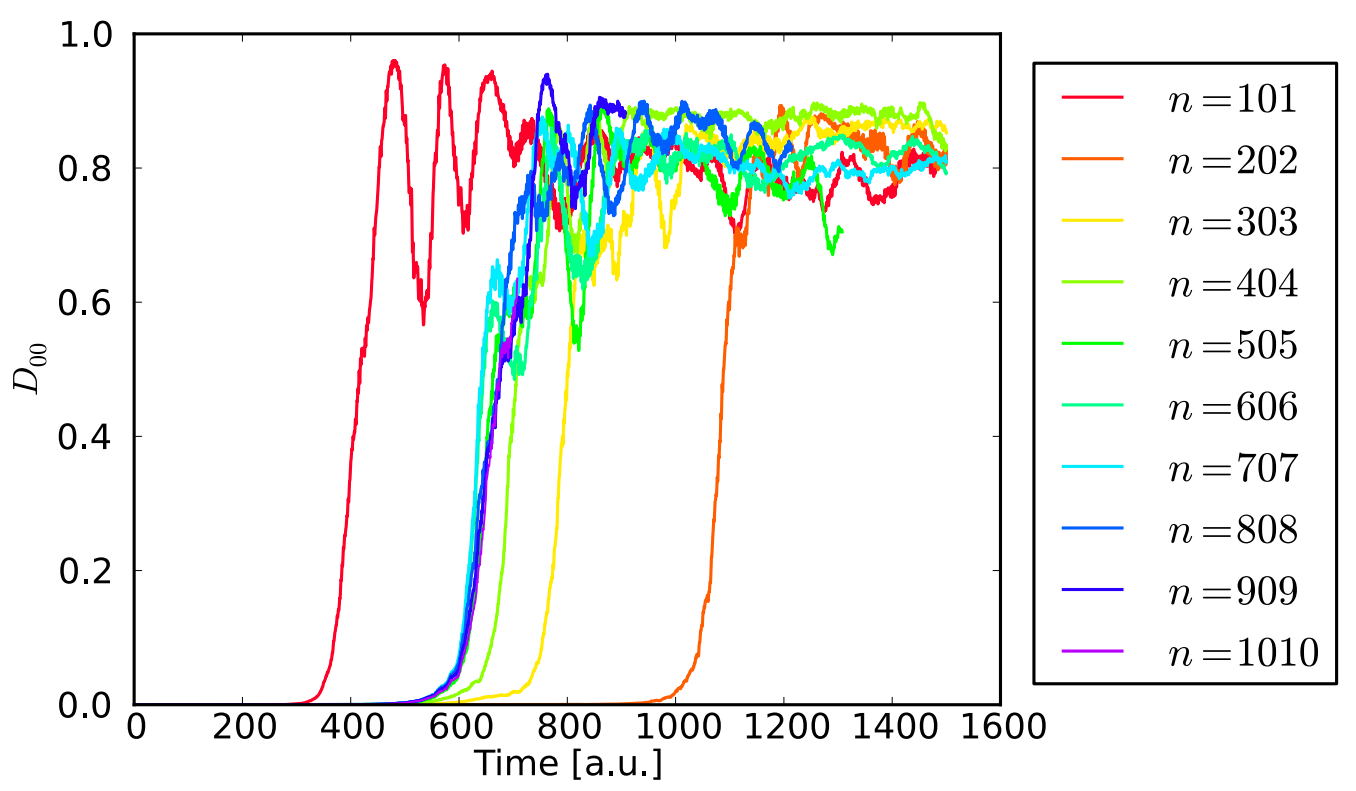

Figure 3.3: $\boldsymbol{D}^{\alpha \prime}{ }_{00}(t)$ is plotted as function of time for various grid sizes. The inset shows the time of transition as a function of the grid size. A timestep of $0.001 \mathrm{a} . \mathrm{u}$. was used for all the simulations.

occupation of the $\chi_{0}^{\alpha}$ orbital increases within about 100 a.u. to about 0.8 a.u. This means that the initially created hole in the core-shell is refilled.

The inset in Figure 3.3 shows the transition time as a function of the grid size. The transition time was determined as the time when $D^{\alpha \prime}{ }_{00}(t)$ has increased to 0.66 a.u. and it converges to about 700 a.u. at 505 grid points. Hence, 505 grid points were used for the simulations.

Then a appropriate time step was derived using a similar approach as above. The dynamics in the six-particle system after removal of a particle from the $\chi_{0}^{\alpha}$ orbital were simulated with different timesteps and a grid size of 101. Like before the transition time, i.e. the time when the core hole is refilled, was applied to compare the different simulations.

Figure 3.4 shows the transition of $\boldsymbol{D}_{00}^{\alpha}(t)$ for different timesteps $\Delta t$ and 101 grid points. The three curves cannot be discerned as they overlay. Hence, the transition time is virtually the same for all timesteps. For the six-particle system the trajectory with $\Delta t=0.001$ a.u. was already available from the grid size determination and, hence, analyzed in the following section. The four-particle system was then also run using the $\Delta t=0.001$ a.u. timestep. 


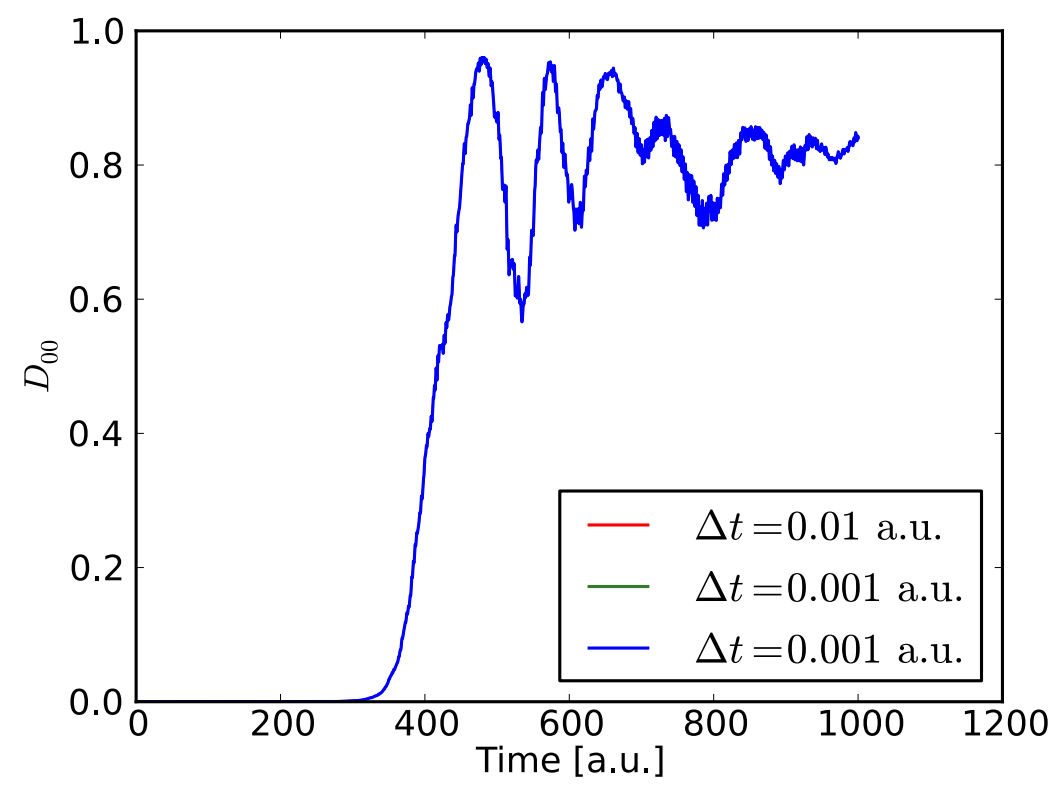

Figure 3.4: Transition in the six-particle system after instantaneous core-shell ionization for different timesteps. Note that the curves are virtually the same and, thus, overlay.

\subsection{Results}

The simple convergence algorithm used (see Algorithm 2.1) only yielded proper initial states for four- and six-particle systems. For systems with more particles the algorithm did not provide ground states that were stable enough for timepropagation. Thus only the four- and the six-particle system are discussed here.

As discussed in the previous section the initial state is prepared by removing a particle from the core-shell $\alpha \mathrm{MO} \chi_{0}^{\alpha}$ of a converged RHF ground state.

In the Auger process a particle from the valence-shell (MO $\left.\chi_{2}\right)$ fills the hole in the core-shell ( $\mathrm{MO} \chi_{0}$ ). In turn, another particle from the valence-shell is emitted into the continuum. The energy of the emitted particle can be estimated as the difference of the energy of the core hole initial state $E^{\text {core }}$ and the energy of the double ionized system $E^{\text {di }}$. If this energy difference it larger then zero, the particle can actually be emitted into the continuum. 


\subsubsection{Four-particle System}

The difference of the energy of the initial state $E^{\text {core }}$ and the doubly ionized state $E^{\mathrm{di}}$ is about $E^{\mathrm{di}}-E^{\mathrm{core}} \approx 0.23 \mathrm{a} . \mathrm{u}$.. Hence, there is enough energy to emit a valence particle into the continuum.
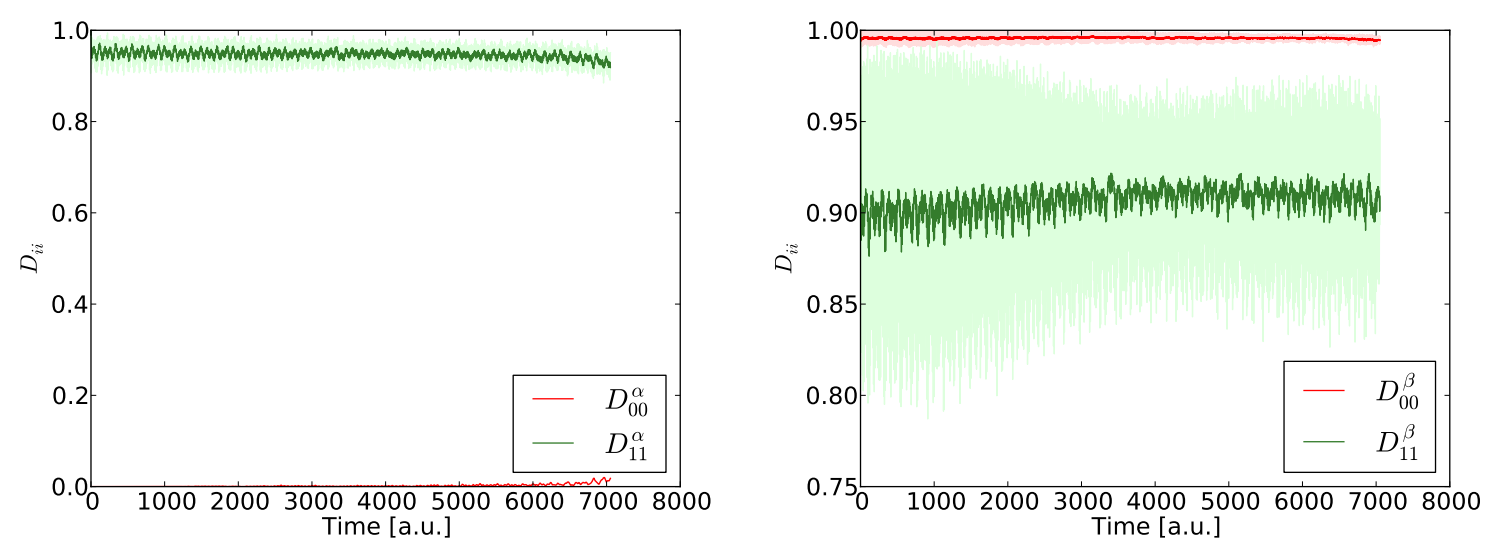

Figure 3.5: Occupation of the initial $\alpha$ (left) and $\beta$ (right) molecular orbitals determined as the corresponding diagonal elements of the one particle reduced density matrix in the space of initial orbitals. The thick line is the running average over 10 a.u..

Figure 3.5 shows the dynamics of the system after the instantaneous removal of a particle from the $\chi_{0}^{\alpha}$ core MO. The occupation of the initial MOs is shown as a function of time. Right after the ionization of the initial $\alpha$ valence MO (left, green line) $\chi_{1}^{\alpha}$ starts to oscillate slightly. The onset of the oscillation is accompanied by a rapid expansion of the $\chi_{1}^{\alpha}(t) \mathrm{MO}$. This expansion is reflected in a rapid increase of the radius of gyration (ROG) right after the ionization (see insets in Figure 3.6, right). Afterwards the extension of $\mathrm{MO} \chi_{1}^{\alpha}(t)$ oscillates around an increasing mean value.

The system shows an immediate reaction to the removal of the particle. There is, however, no transition within the simulation time of about 7000 a.u..

The occupation of the initial $\chi_{1}^{\beta}$ MO (green line) starts to oscillate strongly after the ionization whereas the occupation of core $\chi_{0}^{\beta}$ MO (red line) is barely affected. Similar to the $\alpha$ valence $\mathrm{MO}$ the valence $\beta \mathrm{MO}$ extends rapidly right a the beginning (see inset in Figure 3.6) and oscillates afterwards. 

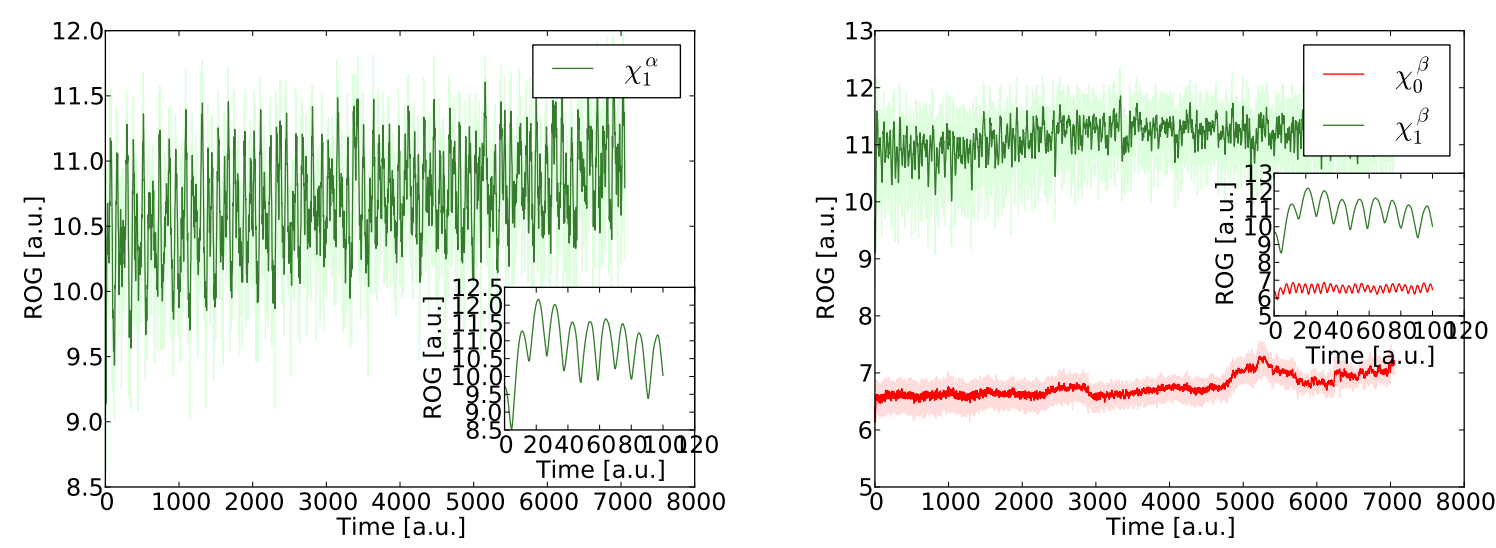

Figure 3.6: The expectation values $\left\langle\chi_{j}^{\alpha}\left|\hat{x}^{2}\right| \chi_{j}^{\alpha}\right\rangle$ are plotted for both the $\alpha$ (left) and $\beta$ (right) molecular orbitals. The thick line is the running average over 10 a.u..

Also the $\beta$ valence orbital shows a rapid reaction to the perturbation. Like in the $\alpha$ case there is no transition observed during this simulation.

The system shows a strong reaction to the removal of the particle. However, no transition occurs during 7000 a.u. in which the core-hole created by the removal of the particle is refilled. Although there is enough energy to emit a valence particle into the continuum, no emission can be observed.

\subsubsection{Six-particle System}

The energy difference is $E^{\mathrm{di}}-E^{\mathrm{core}} \approx 1$.68a.u.. Thus, an Auger-like process is energetically possible.

Figure 3.7 shows the dynamics of the six-particle system after the instantaneous ionization $\left(t=0\right.$ a.u.) of the $\chi_{0}^{\alpha} \mathrm{MO}$ (red line in Figure 3.7 (left)). The occupation of both the $\chi_{2}^{\alpha}$ valence orbital and the $\chi_{2}^{\beta}$ valence orbital starts to oscillate strongly. This oscillation in MO space corresponds to an oscillation in real space. The oscillations in real space are reflected in ROG $\left(\left\langle\chi_{i}\left|\hat{x}^{2}\right| \chi_{i}\right\rangle\right)$ of the valence orbitals (see Figure 3.8 blue lines). Hence, the system immediately reacts to the perturbation of removing a particle.

For about 600 a.u these oscillations of the two valence orbitals are the only visible dynamics. After about 600 a.u. the occupation of the $\chi_{0}^{\alpha}$ MO raises up to 0.9 a.u. within 200 a.u.. At the same time the occupation of both the $\chi_{1}^{\alpha}$ and $\chi_{2}^{\alpha}$ MOs decreases. The occupation of the valence $\chi_{2}^{\alpha}$ MOs drops to essentially zero after 

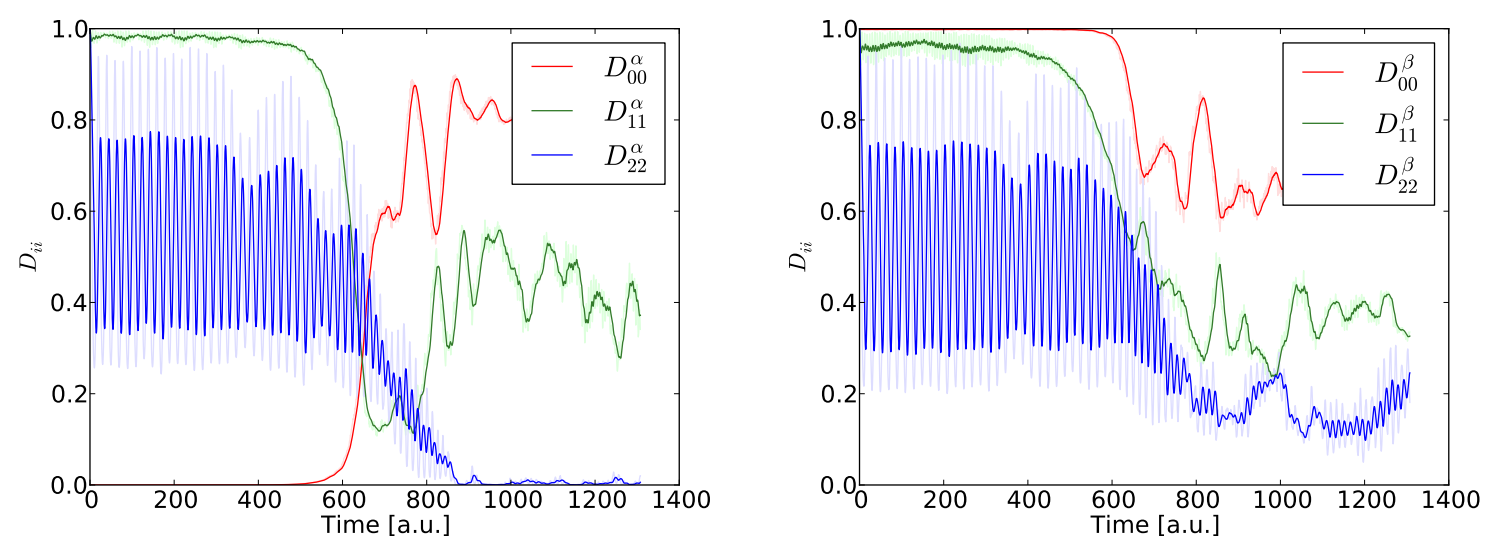

Figure 3.7: Occupation of the initial $\alpha$ (left) and $\beta$ (right) molecular orbitals determined as the corresponding diagonal elements of the one particle reduced density matrix in the space of initial orbitals. The thick line is the running average over 10 a.u..

about 900 a.u.. These changes of the occupations of the initial MO are also reflected in the ROG (see Figure 3.8 (left)) of the two occupied orbitals. The $\chi_{1}^{\alpha}(t) \mathrm{MO}$ (green line) starts to extend significantly at around $500 \mathrm{a} . \mathrm{u}$. while the extension of the $\chi_{2}^{\alpha}(t)$ valence MO (blue line) does a jump around 600 a.u. to 800 a.u..

The observed dynamics indicate a transition taking place in the system. During this transition (600 a.u. -800 a.u.) the initially created core hole is refilled by charge coming from the higher MOs. After this transition the initial valence $\mathrm{MO} \chi_{2}^{\alpha}$ is essentially empty.

Also the $\beta$ MOs show dynamics beyond the oscillation of the valence MO $\chi_{2}^{\beta}$ starting at around 600 a.u.. The occupation of all three initial MOs (see Figure 3.7 (right)) decreases. The occupation of the initial $\chi_{2}^{\beta}$ valence MO (blue line) drops below 0.2 a.u. after about 800 a.u. and does not increase anymore afterwards. The strong oscillation of the occupation of the $\chi_{2}^{\beta}$ MO declines during the transition and essentially vanishes after the occupation has dropped to zero. Similarly the occupation of the initial $\chi_{1}^{\beta}$ MO drops down to about 0.3 a.u. and oscillates around this value afterwards. Also the occupation of the initial $\chi_{0}^{\beta}$ core MO decreases. It drops to o.6 a.u. after about 800 a.u. but increase again afterwards to about o.8 a.u..

Again the change in the occupations of the initial $\mathrm{MO}$ is accompanied by a change of the ROG (see Figure 3.8) of the current MOs. All three $\beta$ MOs expand. 

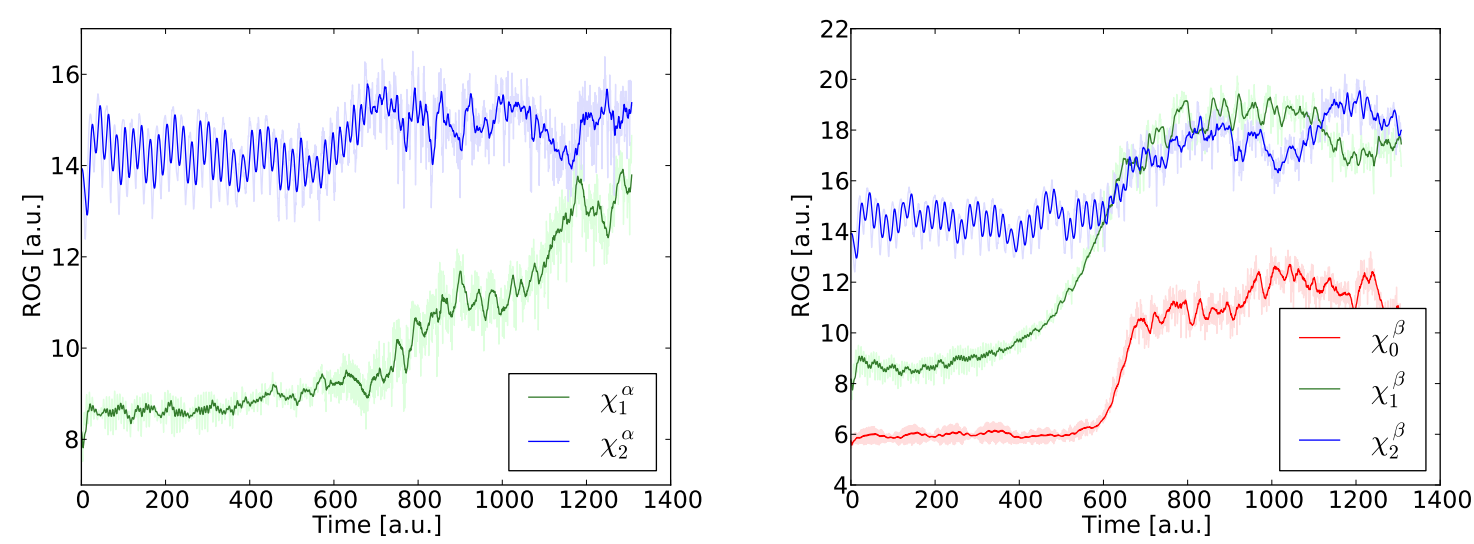

Figure 3.8: The expectation values $\left\langle\chi_{j}^{\alpha}\left|\hat{x}^{2}\right| \chi_{j}^{\alpha}\right\rangle$ are plotted for both the $\alpha$ (left) and $\beta$ (right) occupied molecular orbitals. The thick line is the running average over 10 a.u..

The "middle" $\chi_{1}^{\beta}$ expands gradually during the time frame 400 a.u. -800 a.u. while especially the extension of the $\chi_{0}^{\beta}$ core MO increases rapidly within about 100 a.u..

After the instantaneous removal of a particle from the six-particle ground state a transition occurs in the system in the time interval 6oo a.u.-80oa.u.. During the transition the initial $\alpha$ core hole is refilled while the occupation of the initial $\alpha$ valence $\mathrm{MO}$ decreases to essentially zero. At the same time the occupations of all three $\beta$ MOs decreases. The refilling of the core hole resembles the Auger process. Although there is enough energy available to ionize the valence MO, no clear ionization of the $\beta \mathrm{MO}$ is observed. All three $\beta \mathrm{MOs}$ expand instead.

Despite the lack of a clear emission of a $\beta$ particle into the continuum, the results are still promising. The simulations indicate, that TDHF is capable of describing a process in which an instantaneously create core hole is refilled by the remaining particles. The absence of a clear auto-ionization could still be caused by the specific interactions in this model system. Hence, the methodology used in the chapter to simulate the dynamics in the model system will be transferred to atoms in the following chapter. 


\section{4 \\ Beryllium}

In the previous chapter it was shown that a time-dependent Hartree-Fock (TDHF) based approach answers the purpose of describing the refilling dynamics for the core hole created by instantaneous ionization of a one-dimensional model system. Due to this encouraging result, a similar TDHF was applied to single atoms, to test if TDHF is sufficient to describe the Auger decay in realistic systems.

As atoms are spherically symmetric, they can be seen as one-dimensional because the angular part can be treated by spherical harmonics[59]. Typically this decomposition is directly applied to the orbitals

$$
\chi_{j}(\boldsymbol{r})=Y_{l_{j}}^{m_{j}}(\theta, \phi) \frac{P_{j}(r)}{r}
$$

and then the differential equations for the radial part $P_{j}(r)$ are solved directly[19, 54]. Here, I use a different approach that retains the matrix formulation of Chapter 2.1.2. The orbitals $\chi_{j}(\boldsymbol{r})$ are expanded in a basis set $\mu(\boldsymbol{r})$ which is in turn decomposed in an angular and a radial part

$$
\mu(\boldsymbol{r})=Y_{l_{\mu}}^{m_{\mu}}(\theta, \phi) \frac{R_{\mu}(r)}{r} .
$$

First, the radial part is treated using a numerical grid. The numerical grid has a great computational advantage as the two-electron integrals reduce to a two index quantity greatly reducing the number of two-electron integrals and, thus, the memory requirement. Usually exponential grids are used[19, 54], which allow a good description of the orbitals in the vicinity of the nucleus. However, for the (pseudo-) continuum part a uniform grid is necessary. As general non-uniform grids lead to a non-symmetric representation of the kinetic energy operator[60, 61], a uniform grid is used throughout the whole radial box. 
Second, a B-spline basis[20] is deployed to expand the radial part of the orbitals. B-spline basis sets are utilized successfully in atomic and molecular physics[21, 22]. They offer the advantage of an analytic representation of the kinetic energy operator. Additionally, the representation of the kinetic energy operator is symmetrical irrespective of the choice of the knot sequence. A parabolic-linear knot sequence[21] is employed, which allows for both a good description of the orbitals in the vicinity of the nucleus and the (pseudo-) continuum.

Beryllium is the second lightest atomic species showing Auger decay after Lithium[12]. Beryllium was chosen over Lithium as the test system because it has a closed shell ground state, that simplifies the determination of the initial state. Furthermore, both bound molecular orbitals $(\mathrm{MOs})^{1}$ have $s$ symmetry[62]. As the angular momentum is conserved, also the emitted Auger electron has s symmetry. Thus, only MOs need to be taken into account in the calculation which simplifies the implementation of the working equations and reduces the computational effort.

To my knowledge there is no experimental lifetime of the autoionizing core hole state for atomic Beryllium available. The theoretically determined core hole lifetimes range from $7.0 \mathrm{fs}$ to $32.9 \mathrm{fs}[63-66]$. Therefore, the electron dynamics calculation should, at least cover about $33 \mathrm{fs}$ to test if the TDHF approach predicts the Auger decay for Beryllium in the correct time window.

The photo-ionization is again modeled using the sudden approximation[56] (cf. Section 3.2).

${ }^{1}$ We adopt the notation that a $\mathrm{MO}$ is the solution of the Hartree-Fock (HF) equations even in an atom. This notation is used to avoid possible confusion with the quantum chemistry notation of atomic orbitals (AOs) being basis functions used to expand MOs. 


\subsection{Numerical Hartree-Fock}

As in the previous chapter, the matrix formulation from Section 2.1.2 is retained despite the numerical treatment by defining the "basis"

$$
\begin{aligned}
\mu(\boldsymbol{r}) & =Y_{l_{\mu}}^{m_{\mu}}(\theta, \phi) \frac{R_{\mu}(r)}{r} \mu=1 \cdots N, \\
R_{\mu}(r) & =\left\{\begin{array}{l}
1, r=\mu \Delta, \\
0
\end{array},\right.
\end{aligned}
$$

where the angular part is described by the spherical harmonics $Y_{l_{\mu}}^{m_{\mu}}$. The $\frac{1}{r}$ term in (4.3) ensures the radial part of the MOs $\frac{P_{j}(r)}{r}$ has the right asymptotics at the origin $P_{j}(r \rightarrow 0) \rightarrow 0$.

As the basis (4.3) is orthonormal, the overlap matrix equals the unity matrix $S=\mathbf{1}$. The nuclear potential integrals are given by

$$
\begin{aligned}
\left(\mu\left|V^{\mathrm{nuc}}\right| n u\right) & =\int_{\Omega} \mathrm{d} \omega \int_{0}^{\infty} \mathrm{d} r Y_{l_{\mu}}^{-m_{\mu}}(\omega) Y_{l_{v}}^{m_{v}}(\omega) \delta\left(r-R_{\mu}\right)\left(-\frac{Z}{r}\right) \delta\left(r-R_{v}\right) \\
& =-\delta_{\mu v}\left(\frac{Z}{R_{\mu}}\right),
\end{aligned}
$$

where the integral over the whole space $\int_{\mathcal{R}^{3}} \mathrm{~d} r$ is written as a product of the angular part $\int_{\Omega} \mathrm{d} \omega$ and the radial part $\int_{0}^{\infty} \mathrm{d} r r^{2}$. The kinetic energy operator is approximated by the second order central difference approximation in the radial space

$$
\left(\mu\left|-\frac{1}{2} \Delta\right| v\right)=\delta_{\mu v}-\frac{1}{2}\left(\delta_{\mu(v-1)}+\delta_{\mu(v+1)}\right) .
$$

The two-electron electron integrals are calculated from

$$
\begin{aligned}
(\mu \nu \mid \lambda \sigma) & =\iint_{\mathcal{R}^{3}} \mathrm{~d} \boldsymbol{r}_{1} \boldsymbol{r}_{2} \mu^{*}\left(\boldsymbol{r}_{1}\right) \nu\left(\boldsymbol{r}_{1}\right) \frac{1}{\left|\boldsymbol{r}_{1}-\boldsymbol{r}_{2}\right|} \lambda^{*}\left(\boldsymbol{r}_{2}\right) \sigma\left(\boldsymbol{r}_{2}\right) \\
& =\sum_{l=0}^{\infty} \sum_{m=-l}^{l}(-1)^{m} \sqrt{\left(2 l_{\mu}+1\right)\left(2 l_{v}+1\right)\left(2 l_{\lambda}+1\right)\left(2 l_{\sigma}+1\right)} \\
& \times\left(\begin{array}{ccc}
l_{\mu} & l_{v} & l \\
0 & 0 & 0
\end{array}\right)\left(\begin{array}{ccc}
l_{\mu} & l_{v} & l \\
-m_{\mu} & m_{v} & -m
\end{array}\right)\left(\begin{array}{ccc}
l_{\lambda} & l_{\sigma} & l \\
0 & 0 & 0
\end{array}\right)\left(\begin{array}{ccc}
l_{\lambda} & l_{\sigma} & l \\
-m_{\lambda} & m_{\sigma} & m
\end{array}\right)
\end{aligned}
$$




$$
\times \delta_{R_{\mu} R_{v}} \delta_{R_{\lambda} R_{\sigma}} \frac{r_{<}^{l}}{r_{>}^{l+1}},
$$

where the relation between the spherical harmonics, the Clebsch-Gordan coefficients and the Wigner $3-j$ symbols $[67,68]$ are used together with Laplace expansion[69] of the electron-electron interaction

$$
\frac{1}{\left|\boldsymbol{r}_{1}-\boldsymbol{r}_{2}\right|}=\sum_{l=0}^{\infty} \sum_{m=-l}^{l} \frac{4 \pi}{2 l+1} Y_{l}^{-m}(\theta, \phi) Y_{l}^{m}(\theta, \phi) \frac{r_{<}^{l}}{r_{>}^{l+1}} .
$$

With the above relations a simple self-consistent field (SCF) procedure was implemented (see Section 2.1.2) to determine the initial state for the electron dynamics. The TDHF equations are solved using the constant mean field (CMF) algorithm discussed in section 2.1.2. 


\subsubsection{Setup}

For the numerical HF calculations two parameters, the grid size and the width of the radial box, need to be chosen. The grid size $N$ determines the computational effort for the dynamics simulations and cannot be chosen arbitrarily large. The grid size $N$ and the box width define the spectrum of the MOs. The energy of the bound MOs is mainly defined by the quality of the respective $\mathrm{MO}$ in the vicinity of the nucleus. For a good description a small grid spacing is needed. The continuum part of the orbital spectrum is dominated by the spectrum of the kinetic energy operator

$$
\epsilon_{n}=\frac{2}{(\Delta x)^{2}}\left(1-\cos \left(\frac{\pi n}{2 N}\right)\right),
$$

with the grid spacing $\Delta x$. Thus, the continuum part of the spectrum mostly covers a high energy part if the grid spacing is too small. Hence, two trade-offs had to be made, between the computational effort and the quality of the MOs as well as between the bound orbital energies and the energy range covered by the continuum spectrum.

The grid size had been chosen such that the expected core-hole lifetime was covered by the simulation time with reasonable computational effort. The grid size was picked to be 1000, which yielded a simulation time of about $35 \mathrm{fs}$ with about 4 weeks of computer time.
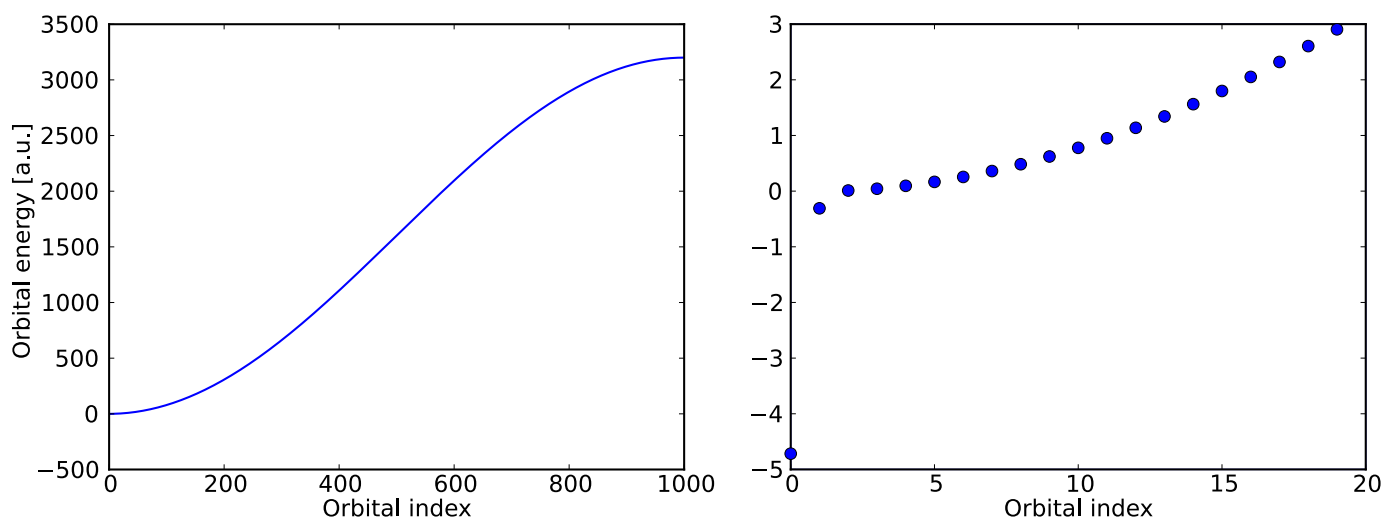

Figure 4.1: Spectrum of the ground state Fock matrix for 1000 grid points and a radial box size of $25 a_{0}$. 
The box width was then chosen to give a balance between the energies of the bound MOs which also determine the ground state HF energy and the energy range covered by the continuum spectrum.

The width of the radial box was set to $25 a_{0}$. This yields a ground state restricted Hartree-Fock (RHF) energy of $E^{0} \approx-14.54$ a.u., which reproduces the ground state RHF energy from the literature[19] to $10^{-3}$ a.u.. The spectrum of the ground state RHF Fock operator is shown in Figure 4.1. The continuum part of the spectrum unfortunately still covers a wide uninteresting energy range $>10$ a.u., but further increasing the grid spacing would diminish the energies of the MOs. Hence, the parameters described above, grid size of $N=1000$ and a box size of $25 a_{0}$ were used in the dynamics simulation.

\subsubsection{Results}

The energy difference between the initial core-hole and the doubly ionized state was found to be about 3.8a.u.. Since the energy difference is positive, there is enough energy available to ionize the valence $\mathrm{MO}$ and the Auger decay is energetically possible.


Figure 4.2: The expectation values $\left\langle\chi_{j}^{\alpha}\left|\hat{x}^{2}\right| \chi_{j}^{\alpha}\right\rangle$ are plotted for both the $\alpha$ (left) and $\beta$ (right) molecular orbitals. The thick line is the running average over approximately 40 a.u..

Figure 4.2 shows the time-evolution of the radius of gyration (ROG) $\left\langle\chi_{j}^{\alpha}\left|\hat{x}^{2}\right| \chi_{j}^{\alpha}\right\rangle$ of the occupied orbitals after the ionization of the $\alpha$ core MO. Right after the ionization, the two valence MO $\chi_{1}^{\sigma}$ (green lines) (see Figure 4.2 insets) first quickly 
contract and then expand. In addition of the expansion, the extension of the valence MOs starts to oscillate. This oscillation extends over the whole simulation.

Also the $\beta$ core $\mathrm{MO}$ (red line) extends right after the ionization. After the initial expansion (about $0.2 \mathrm{fs}$ ), the extension of the $\chi_{0}^{\beta}$ core MO stays essentially constant for the rest of the simulation.
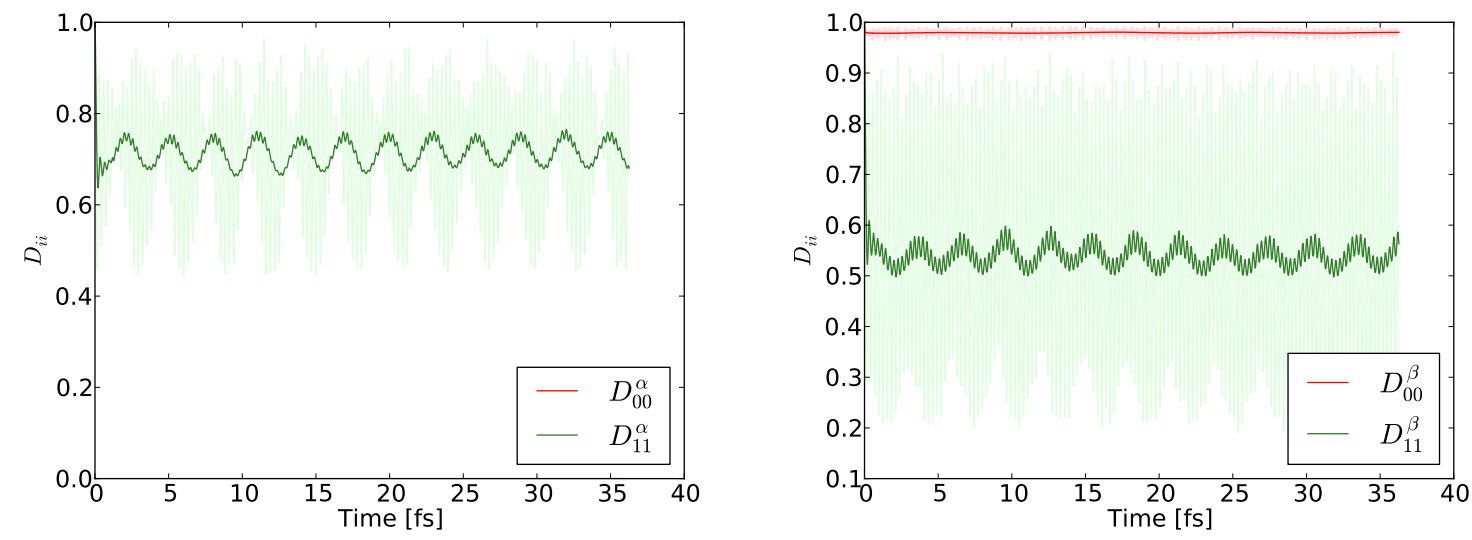

Figure 4.3: Occupation of the initial $\alpha$ (left) and $\beta$ (right) molecular orbitals determined as the corresponding diagonal elements of the one particle reduced density matrix in the space of initial orbitals. The thick line is the running average over approximately 40 a.u..

This dynamics are also reflected in the occupation of the initial MO (see Figure 4.3). The occupation of both valence MO (green lines) drop right after the ionization. The occupation of the initial $\chi_{1}^{\alpha} \mathrm{MO}$ decreases to about $0.7 e^{-}$. After the initial drop, the occupation of the initial $\chi_{1}^{\alpha}$ oscillates around that value. Similarly, the occupation of the initial $\chi_{1}^{\beta}$ decreases to about $0.55 e^{-}$and oscillates strongly around that value with an amplitude of about $0.3 e^{-}$. The occupation of the initial $\chi_{0}^{\beta}$ core MO decreases slightly after the ionization. Afterwards, the occupation of the $\chi_{0}^{\beta}$ core MO stays essentially constant until the end of the ionization.

There is no increase in the occupation of the initial $\chi_{0}^{\alpha}$ MO. Accordingly, no transition observed within the simulation time of about $37 \mathrm{fs}$. Thus, the numerical HF does not describe the Auger decay within the expected time window (about $33 \mathrm{fs}$ ). The lack of the transition could simply be caused by the limitations of the HF theory, especially the limited description of electron correlation. On the other hand, the approach used above has additional limitations. Especially the 
kinetic energy operator is approximated by second order differencing and the grid describes a continuum spectrum that mostly covers an energy range that is way above the expected energy of the Auger electron.

To exclude possible influence of the grid basis on the electron dynamics and to judge the applicability of TDHF theory to the Auger process, a B-spline basis was employed the following section. 


\subsection{B-spline Hartree-Fock}

In the previous section it was shown that the TDHF theory with a numerical grid is probably not capable of describing the Auger process properly. To make sure that this limitation is caused by the HF approximations and not by the numerical grid used, a B-spline[20] basis set was used together with TDHF theory in a similar setting as before.

The B-spline basis offers several advantages over the numerical grid. It allows an analytic expression of all electron operators, especially the kinetic energy operator. Furthermore, a B-spline basis together with a non-uniform knot sequence allows a balanced description of both the bound and continuum MOs and, thus, a compacter basis offering a higher computational efficiency.

A B-spline ${ }^{1}$ basis set $B_{i, K}^{\tau}$ is employed as the radial part of the basis

$$
\mu(\boldsymbol{r})=Y_{l_{\mu}}^{m_{\mu}}(\theta, \phi) \frac{B_{i_{\mu}, K}^{\tau}(r)}{r}, \mu=1 \cdots N
$$

and is defined within the computational box $[0, R]$. The $K$ th order B-splines are determined by the recursion relation with respect to the knot sequence $\tau=\left\{\tau_{i}\right\}_{i=1, \ldots, M}$

$$
\begin{aligned}
B_{i, K}^{\tau}(x) & =\frac{x-\tau_{i}}{\tau_{i+K-1}-\tau_{i}} B_{i, K-1}^{\tau}+\frac{\tau_{i+K}-x}{\tau_{i+K}-\tau_{i+1}} B_{i+1, K-1}^{\tau}, \\
B_{i, 1}^{\tau}(x) & = \begin{cases}1, & \tau_{i} \leq x<\tau_{i} \\
0, & \text { else }\end{cases}
\end{aligned}
$$

So the $K$ th order B-splines $B_{i, K}^{\tau}$ are polynomials of order $K-1$ with compact support $\left[\tau_{i}, \tau_{i+K}\right]$. The derivative of the B-splines are given by[20]

$$
\left(B_{i, K}^{\tau}\right)^{\prime}(x)=\frac{K-1}{\tau_{i+K-1}-\tau_{i}} B_{i, K-1}^{\tau}(x)-\frac{K-1}{\tau_{i+K}-\tau_{i+1}} B_{i+1, K-1}^{\tau}(x) .
$$

It is principally possible to derive analytic formulas for the various one- and twoelectron integrals needed in Hartree-Fock calculations. This is, however, tedious and does not necessarily yield efficient implementations. Instead Gauss-Legendre quadrature is used to evaluate the integrals over the intervals $\left[\tau_{i}, \tau_{i+1}\right]$. The Gauss-

\footnotetext{
${ }^{1}$ In the following, B-spline(s) will denote both the individual function and the set of functions, which is defined by the order $K$ and the knot sequence $\tau$.
} 
Legendre quadrature of order $N_{G L Q}=2 \mathrm{~K}-2$ is able to exactly integrate the overlap matrix elements

$$
\begin{aligned}
S_{\mu v} & =(\mu \mid v)=\int_{\Omega} \mathrm{d} \omega Y_{l_{\mu}}^{-m_{\mu}}(\omega) Y_{l_{v}}^{m_{v}}(\omega) \int_{0}^{R} \mathrm{~d} r B_{i_{\mu}, K}^{\tau}(r) B_{i_{v}, K}^{\tau}(r) \\
& =\delta_{l_{\mu} l_{v}} \delta_{m_{\mu} m_{v}} \int_{0}^{R} B_{i_{\mu}, K}^{\tau} B_{i_{v}, K}^{\tau}
\end{aligned}
$$

and the elements of the representation of the kinetic energy operator

$$
\begin{aligned}
T_{\mu v} & =-\frac{1}{2}(\mu|\Delta| v) \\
& -\frac{1}{2} \int_{\Omega} \mathrm{d} \omega \int_{0}^{R} \mathrm{~d} r Y_{l_{\mu}}^{-m_{\mu}}(\omega) B_{i_{\mu}, K}^{\tau}\left(\frac{\partial}{\partial r}\left(r^{2} \frac{\partial}{\partial r}\right)-\frac{1}{r^{2}} \hat{L}^{2}\right) Y_{l_{v}}^{m_{v}}(\omega) B_{i_{v}, K}^{\tau} \\
& =\frac{1}{2} \delta_{l_{\mu} l_{v}} \delta_{m_{\mu} m_{v}} \int_{0}^{R} \mathrm{~d} r\left(B_{i, K}^{\tau}\right)^{\prime}(r)\left(B_{i, K}^{\tau}\right)^{\prime}(r)+\frac{l_{\mu}\left(l_{\mu}+1\right)}{r^{2}} B_{i_{\mu}, K}^{\tau}(r) B_{i_{v}, K}^{\tau}(r),
\end{aligned}
$$

as the integrated functions are polynomials of order $\leq 2 K-2$. The potential integrals cannot be integrated exactly by Gauss-Legendre quadrature as the integrands are rational functions rather then polynomials. In practice the potential integrals converge rapidly to machine precision with increasing order of the quadrature[21]. The nuclear attraction integrals

$$
\begin{aligned}
V_{\mu v}^{\text {nuc }} & =\int_{\Omega} \mathrm{d} \omega Y_{l_{\mu}}^{-m_{\mu}}(\omega) Y_{l_{v}}^{m_{v}}(\omega) \int_{0}^{R} \mathrm{~d} r \frac{1}{r} B_{i_{\mu}, K}^{\tau}(r) B_{i_{v}, K}^{\tau}(r) \\
& =\delta_{l_{\mu} l_{v}} \delta_{m_{\mu} m_{v}} \int_{0}^{R} \mathrm{~d} r \frac{1}{r} B_{i_{\mu}, K}^{\tau}(r) B_{i_{v}, K}^{\tau}(r)
\end{aligned}
$$

can simply be evaluated with the increased order quadrature. The two-electron integrals

$$
\begin{aligned}
(\mu \nu \mid \lambda \sigma) & =\sum_{l=0}^{\infty} \sum_{m=-l}^{l}(-1)^{m} \sqrt{\left(2 l_{\mu}+1\right)\left(2 l_{v}+1\right)\left(2 l_{\lambda}+1\right)\left(2 l_{\sigma}+1\right)} \\
& \times\left(\begin{array}{ccc}
l_{\mu} & l_{v} & l \\
0 & 0 & 0
\end{array}\right)\left(\begin{array}{ccc}
l_{\mu} & l_{\nu} & l \\
-m_{\mu} & m_{\nu} & -m
\end{array}\right)\left(\begin{array}{ccc}
l_{\lambda} & l_{\sigma} & l \\
0 & 0 & 0
\end{array}\right)\left(\begin{array}{ccc}
l_{\lambda} & l_{\sigma} & l \\
-m_{\lambda} & m_{\sigma} & m
\end{array}\right)
\end{aligned}
$$




$$
\times \iint_{0}^{R} \mathrm{~d} r_{1} \mathrm{~d} r_{2} B_{i_{\mu}, K}^{\tau}\left(r_{1}\right) B_{i_{v}, K}^{\tau}\left(r_{2}\right) \frac{r_{<}^{l}}{r_{>}^{l+1}} B_{i_{\lambda}, K}^{\tau}\left(r_{2}\right) B_{i_{\sigma}, K}^{\tau}\left(r_{2}\right)
$$

are calculated using the cell algorithm[7o] for the moment integrals

$$
R_{\mu v, \sigma \lambda}^{l}=\iint_{0}^{R} \mathrm{~d} r_{1} \mathrm{~d} r_{2} B_{i_{\mu}, K}^{\tau}\left(r_{1}\right) B_{i_{v}, K}^{\tau}\left(r_{2}\right) \frac{r_{<}^{l}}{r_{>}^{l+1}} B_{i_{\lambda}, K}^{\tau}\left(r_{2}\right) B_{i_{\sigma}, K}^{\tau}\left(r_{2}\right) .
$$

The evaluation of the B-splines and the calculation of the Gauss-Legendre points and weights is implemented using the GNU scientific library[71]

\subsubsection{Setup}

As the first and the last B-splines are non-zero at the origin and the boundary of the box, they are excluded from the calculation to enforce the boundary conditions $P_{j}(r \rightarrow 0)=P_{j}(r \rightarrow R)=0$ yielding $N=M-2$ B-splines as basis functions.



Figure 4.4: Eigenvalue spectra of the converged closed shell Fock matrix with a Bspline basis of order $K=9, N=100$ and a linear-parabolic sequence with $i_{0}=10$ for different box sizes. 


\begin{tabular}{cc}
\hline$R$ & 75 a.u. \\
$N$ & 100 \\
$K$ & 9 \\
$N_{G L Q}$ & 10 \\
\hline
\end{tabular}

Table 4.1: Parameters of the B-spline calculations.

A knot sequence with $K$-fold multiplicity for the first and the last knot is employed

$$
\begin{aligned}
& \tau_{i}=0, \quad i=1, \ldots, K \\
& \tau_{i}=R, \quad i=M, \ldots, M+K .
\end{aligned}
$$

The interior knots have multiplicity one and are chosen according to a linearparabolic sequence[21]

$$
\begin{aligned}
r_{0} & =\frac{R\left(i_{0}-1\right)}{2 M-i_{0}-1}, \\
\alpha & =\frac{r_{0}}{\left(i_{0}-1\right)^{2}}, \\
\beta & =\frac{R-r_{0}}{M-i_{0}}, \\
\tau_{i} & =\left\{\begin{array}{ll}
\alpha(i-K-1)^{2}, & K+1 \leq i<i_{0}+K \\
r_{0}+\beta\left(i-K-i_{0}\right), & i_{0}+K \leq i \leq M
\end{array} .\right.
\end{aligned}
$$

This sequence allows a balanced description of both the bound orbitals, due to the high knot density in the vicinity of the nucleus, and the continuum orbitals by virtue of the linear knot sequence in the rest of the box.

Figure 4.4 shows the eigenvalue spectra of the converged closed shell Fock matrix for different box sizes $R$. The size of the B-spline basis was fixed to $N=100$, which is computationally well accessible. The Auger electron is expected to have an energy of less than 5 a.u.[72] and the grid resulting from a box size of $R=75$ a.u. describes the interesting energy range $<10 \mathrm{a}$.u. well. The parameters from Table 4.I yield a ground state RHF $E^{0}=-14.573023$ a.u., which reproduces the ground state RHF energy from the literature[19] within $10^{-8}$. The parameters summarized in Table 4.1 are thus used in the following. 


\subsubsection{Results}

As in the numerical HF calculation, the difference between the initial core-hole state and the doubly ionized state is about 3.8 a.u.. Again, the energy difference is positive and the Auger decay is energetically allowed.
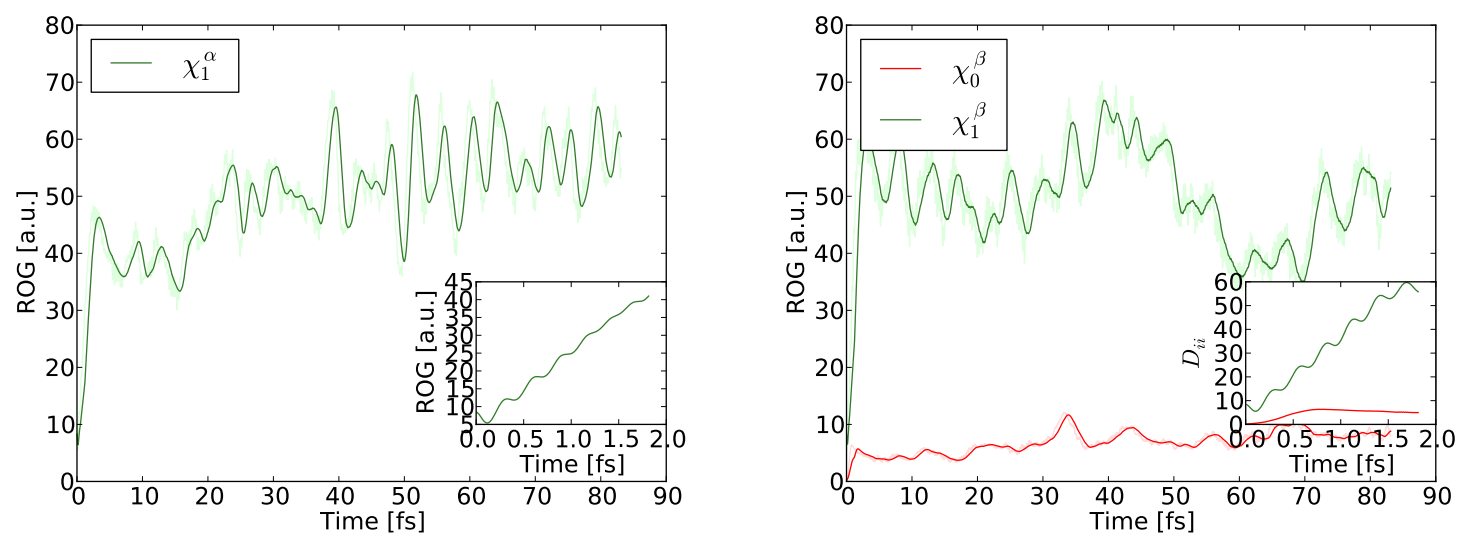

Figure 4.5: The expectation values $\left\langle\chi_{j}^{\alpha}\left|\hat{x}^{2}\right| \chi_{j}^{\alpha}\right\rangle$ are plotted for both the $\alpha$ (left) and $\beta$ (right) molecular orbitals. The thick line is the running average over approximately 40 a.u..

Figure 4.5 shows the ROG $\left\langle\chi_{j}^{\alpha}\left|\hat{x}^{2}\right| \chi_{j}^{\alpha}\right\rangle$ of all three occupied MOs. Like in the numerical HF case, both initial valence MOs $\chi_{1}^{\sigma}$ (green lines) contract right after the ionization ( $t=0 \mathrm{fs}$ ) (see insets). After the initial contraction both valence MOs expand quickly within $2.0 \mathrm{fs}$. After this expansion the extension of the two valence MOs oscillates. Note that the valence MOs expand three times as much as in the numerical HF case. In addition to the oscillation, the $\alpha \mathrm{MO} \chi_{1}^{\alpha}$ expands slowly during the time-evolution. Also, the core $\beta$ MO $\chi_{0}^{\beta}$ expands after the ionization. After that, it oscillates slightly for the rest of the simulation time.

Figure 4.6 shows the occupation of the initial MOs. The occupation of the initial $\chi_{1}^{\sigma}$ MOs decreases rapidly right after the ionization. The occupation of the initial $\alpha$ valence $\mathrm{MO} \chi_{1}^{\alpha}$ drops to $0.82 e^{-}$and oscillates around that value with an amplitude of $0.05 e^{-}$. At that time the occupation of the initial $\beta \mathrm{MO} \chi_{1}^{\beta}$ drops down to $0.72 e^{-}$. After that initial decrease the occupation of the $\chi_{1}^{\beta} \mathrm{MO}$ oscillated strongly with an amplitude of about $0.2 e^{-}$. The occupation of the initial $\chi_{0}^{\beta}$ core MO decreases 

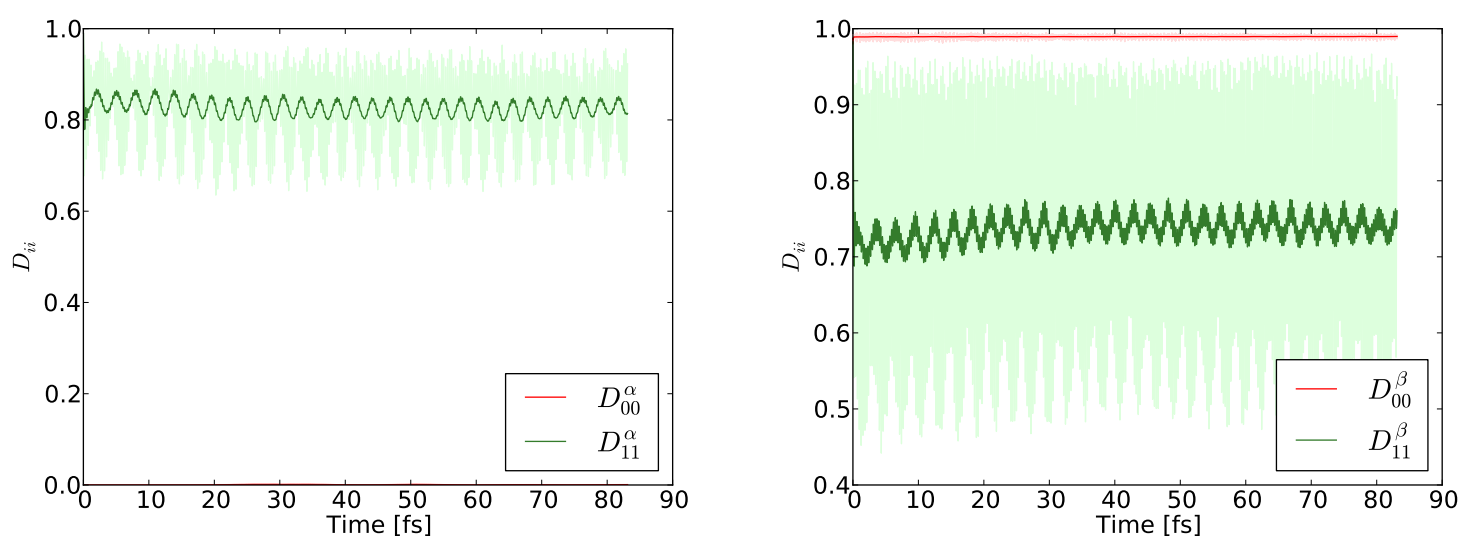

Figure 4.6: Occupation of the initial $\alpha$ (left) and $\beta$ (right) molecular orbitals determined as the corresponding diagonal elements of the one particle reduced density matrix in the space of initial orbitals. The thick line is the running average over approximately 40 a.u..

slightly directly after the ionization and stays essentially constant for the rest of the simulation.

Again no transition, i.e. no refilling of the initial $\chi_{0}^{\alpha}$ core $\mathrm{MO}$ (see Figure 4.6 (left)), is observed. As in the numerical HF simulations no Auger decay can be observed during the simulation.

Despite the better description of the continuum by the B-spline basis compared to the numerical grid, it still offers only a discrete set of MOs in a certain energy region. It cannot be excluded that the density of these discrete states is not high enough for the description of the Auger decay. Another problem arises from the choice of the box width. A slight change of the box width will shift the energy of the continuum MOs. Thus, a particular choice of the box might yield a set of continuum MOs for which the energy of the Auger electron lies between the energy of two continuum MOs causing a poor description of the wavefunction of the Auger electron. It is possible to exclude the second problem by systematically varying the box width within a certain range[21]. 


\section{5 \\ Molecules}

In the previous chapter we have seen that time-dependent Hartree-Fock (TDHF) theory is probably not capable of describing the Auger decay properly due to the limited description of electron correlation within TDHF theory. For further investigations of this limitation, the electron dynamics in two small biomolecules after valence-shell ionization will be studied in this chapter.

Glycine and 2-phenylethyl-N,N-dimethylamine (PENNA) were chosen as test systems as the electron dynamics after instantaneous valence ionization have been described by correlated methods[73, 74], so it is possible to compare the dynamics predicted by TDHF theory to the electron dynamics obtained with a more accurate method.

As we are in general interested in biomolecules, Glycine $\left(\mathrm{H}_{2} \mathrm{NCH}_{2} \mathrm{COOH}\right)$ was chosen as a prototype for peptides. As the smallest amino acid it is furthermore computationally well accessible.

The charge migration dynamics after the ionization from two different molecular orbital (MO) have been characterized by Kuleff et. al.[73] using a correlated ab initio method (non-Dyson algebraic diagrammtic construction (ADC)(3)[75]). To enable a direct comparison to these results a similar computational setup was used, especially the dynamics after ionization from the same MOs were considered (cf. Section 5.3).

PENNA was chosen as a second test system because it exhibits very specific electron dynamics after instantaneous ionization of the highest occupied molecular orbital (HOMO) allowing an easy comparison of the results of different computational approaches.

In addition to the charge migration process predicted by Lünnemann et. al.[74] with the same correlated method as in the Glycine case, it was shown that timedependent density functional theory (TDDFT), using a constant Kohn-Sham matrix 
for the propagation of the MOs, is not sufficient to describe the charge migration process[76].

The availability of different results from the literature enables us to compare our TDHF based approach to a more accurate, correlated method as well as to a less accurate method.

As in the previous chapters the initial states are prepared by removing an electron instantaneously from an $\mathrm{MO}$, describing the photo-ionization process in the sudden approximation[56] (cf. Section 3.2).

\subsection{Implementation}

The most complex part and one of the computational bottlenecks of a HartreeFock (HF) calculation using gaussian basis sets[77] is the integral code used to calculate the two-electron integrals. For an efficient and stable implementation the capabilities of the Gaussian 03 program package[78] were used. The integrals as well as the ground state HF were calculated using the Gaussian 03 program for the following TDHF calculation. The TDHF equations (2.13) were solved with the constant mean field (CMF) algorithm 2.2.

\subsection{Charge Migration Analysis}

To compare the electron dynamics to the results from the literature, the charge migration analysis (CMA) is employed[24, 79]. To this end, the difference density $Q(\boldsymbol{r}, t)$ is defined as the difference between the ground state $\rho_{0}(\boldsymbol{r})$ and the density of the time-evolving state $\rho(\boldsymbol{r}, t)$

$$
Q(\boldsymbol{r}, t)=\rho_{0}(\boldsymbol{r})-\rho(\boldsymbol{r}, t) .
$$

The difference density $Q(\boldsymbol{r}, t)$ can be written as[24]

$$
Q(\boldsymbol{r}, t)=\sum_{p q} \chi_{p}^{*}(\boldsymbol{r}) \chi_{q}(\boldsymbol{r}) N_{p q}(t)
$$

with the time-dependent hole density matrix given for HF wave functions

$$
N_{p q}(t)=\delta_{p q} n_{p}-\left(C_{0}^{\dagger} S D(t) S C\right)_{p q}{ }^{\prime}
$$


where $n_{p}$ are the orbital occupations in the closed shell ground state. Analogous to normal density matrices[8o] the diagonal elements of the hole density matrices can be interpreted as the fraction to which the hole occupies the respective initial MO. For the CMA the hole density matrix $N(t)$ is diagonalized to obtain the natural charge orbitals and the hole occupation numbers[24]

$$
Q(\boldsymbol{r}, t)=\sum_{p}\left|\tilde{\chi}_{p}(\boldsymbol{r}, t)\right|^{2} \tilde{n}_{p}(t) .
$$

\subsection{Glycine}

The structure of the glycine molecule was obtained from a geometry optimization with the Gaussian 03 program package using the DZP[78] basis set $^{1}$. The DZP basis was used in accordance with Reference [73]. Figure 5.1 shows the optimized structure of Glycine (the exact coordinates are given in the Appendix A.1) and the 11th (blue surface) and 16th (red surface) MOs which are subject to the ionization. The 11th and 16th are chosen for ionization to enable a direct comparison of the electron dynamics obtained in this work with the electron dynamics obtained in Reference [73].

The ground state geometry has a symmetry plane which contains all atoms except the hydrogen atoms in the $\mathrm{NH}_{2}$ and $\mathrm{CH}_{2}$ groups. The structure, thus, belongs to the symmetry group $C_{s}$. Hence, the MOs are either symmetric or anti-symmetric with respect to this symmetry plane.

The DZP basis was also used for the following TDHF calculation.

\subsubsection{Ionization from 11th Molecular Orbital}

As discussed above, the ionization from the 11th MO (see Figure 5.2 top left) is considered first. The 11th $\mathrm{MO}$ is located on the $\mathrm{CO}$ and $\mathrm{OH}$ groups on the opposing site of the amine group. It is symmetric with respect to the symmetry plane of the glycine molecule.

Figure 5.2 shows the time-evolution of the electronic wavefunction after the ionization of the 11th MO. The diagonal elements of the hole density matrix (see Equation (5.3)) are plotted.

${ }^{1}$ As obtained from the Environmental Molecular Sciences Laboratory (EMSL) basis set exchange[81, 82]. 


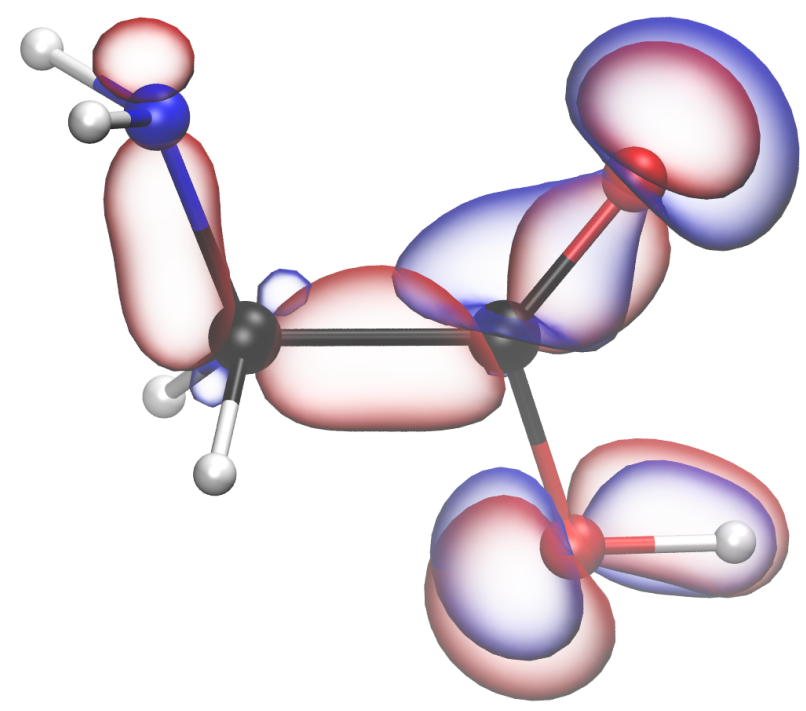

Figure 5.1: Hartree-Fock minimum structure of the glycine molecule in the DZP basis. The isosurfaces show the 11th molecular orbital (blue) and the 16th molecular orbital (red), which are ionized to generate the initial structure. Both are symmetric with respect to the symmetry plane of the molecule.

Right after the ionization the hole occupies only the 11th MO (black line). Then part of the hole is transferred to the 15th (blue line) and the 16th (yellow line) MO. The hole occupation of the 15th MO oscillates with a period of about $2 \mathrm{fs}$. After about $2 \mathrm{fs}$, also the 13th MO (green line) gains hole occupation. At about $3 \mathrm{fs}$ the occupation of the $13^{\text {th }} \mathrm{MO}$ has increased to about $0.4 e$. The hole oscillates once between the 13th and the 11th MO. The 16th MO (yellow) is spread over the whole molecule while the $15^{\text {th }} \mathrm{MO}$ (blue) is mostly located on the amine end of the molecule. Thus, about $0.15 e$ is transferred from one end of the molecule (11th $\mathrm{MO}$ ) to the other end (16th $\mathrm{MO}$ ) in about 500 as. In contrast, the charge exchange between the 11th $\mathrm{MO}$ (black) and 13th MO (green) takes places mostly on the carboxyl group because both MOs are located there.

After about 9 fs the hole occupies the 1oth, 11th, 13th, 15th, 16th and 19th MO. The occupation of the initially ionized 11th MO has a short peak around $10.5 \mathrm{fs}$. After $12 \mathrm{fs}$, the occupation of the 11 th $\mathrm{MO}$ has dropped below $0.1 \mathrm{e}$. At the end of the simulation the hole is spread over all six MOs considered here, especially the hole occupation of the initially ionized MO 11 (black) has dropped below $0.1 e$. 


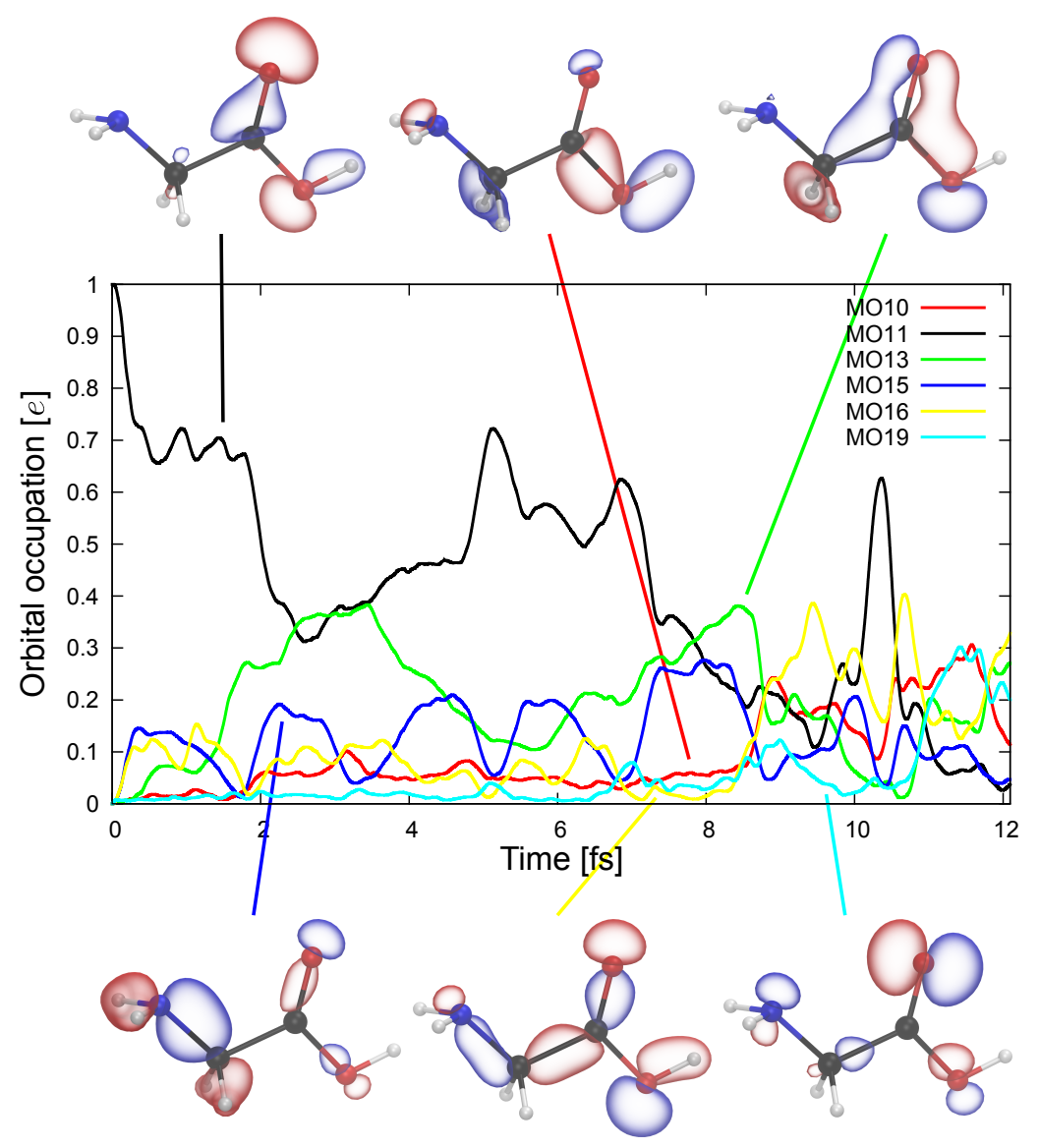

Figure 5.2: The time-evolution of the Hartree-Fock wavefunction of ionization from the 11th molecular orbital. The diagonal elements of the time-dependent hole density matrix are plotted.

Note that the hole only occupies $\mathrm{MO}$ that are symmetric with respect to the symmetry plane of the glycine molecule. Thus, the initial symmetry of the hole is preserved during the time-evolution of the electronic wavefunction.

The change of the occupation of the initial $\beta$ MOs is less than $0.1 e$. In contrast to the core-shell ionization considered in the previous chapter the perturbation of removing a valence electron seems small, so the $\beta$ MOs show only little dynamics.

The electron dynamics after $8 \mathrm{fs}$ resembles a diffusion of the hole in the space of symmetric valence MOs without the HOMO (which is the 2oth $\mathrm{MO}$ ). This picture is supported by the fact that no dynamics of the $\beta$ MOs are observed. 


\subsubsection{Ionization from 16th Molecular Orbital}

Now the ionization from the 16th MO (see Figure $5 \cdot 3$ (top left)) is considered. The 16 th $\mathrm{MO}$ is distributed among the whole molecule. Also the 16 th $\mathrm{MO}$ is symmetric with respect to the symmetry plane of the glycine structure.

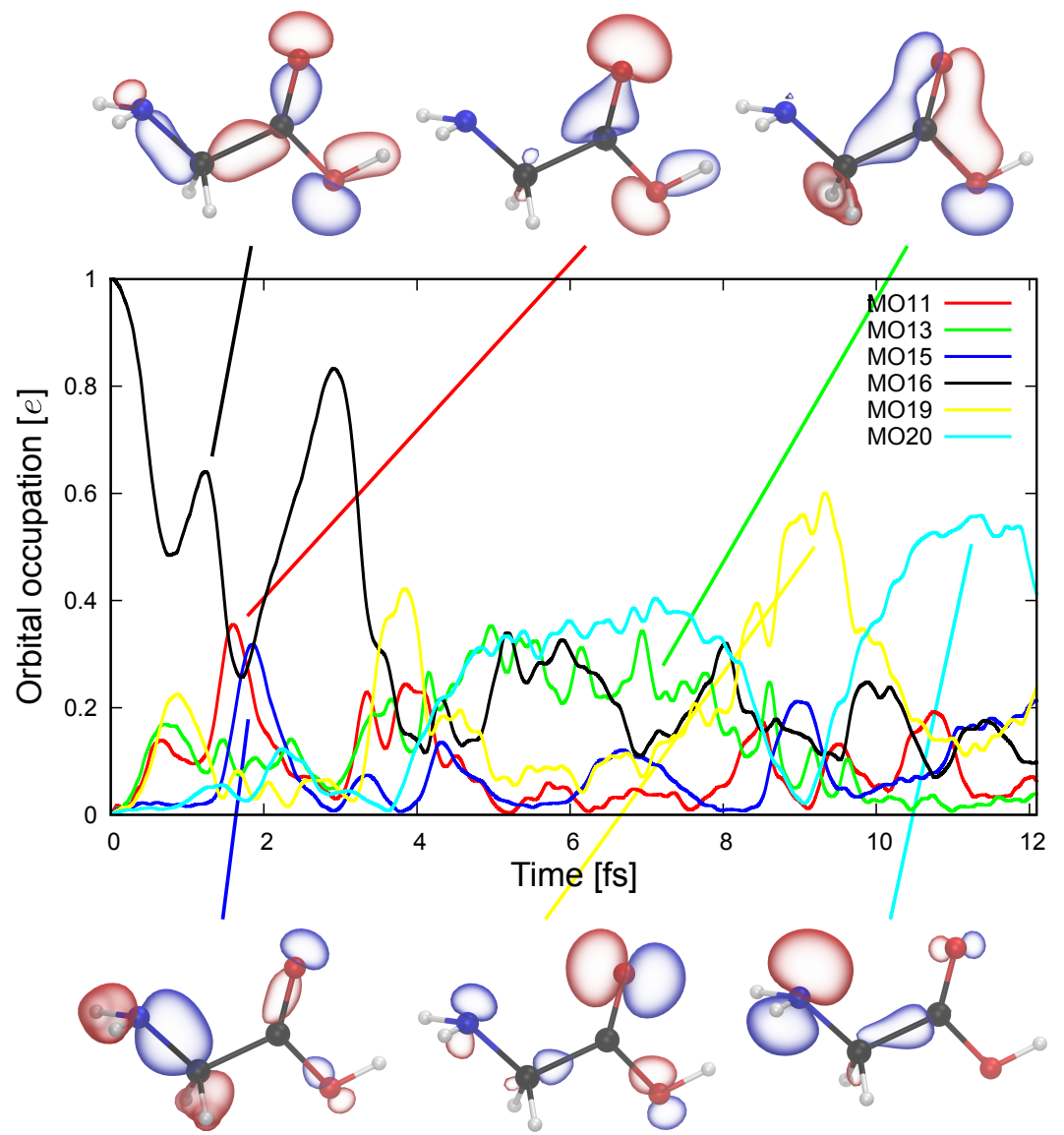

Figure 5.3: The time-evolution of the Hartree-Fock wavefunction of ionization from the 16th molecular orbital. The occupations of the relevant initial orbitals are shown.

Figure 5.3 shows the time-evolution of the electronic wavefunction after the ionization of the 16th MO. Again, the diagonal elements of the hole density matrix (see Equation (5.3)) are plotted.

The occupation of the initially occupied MO 16 (black line) decreases to $0.5 e$ within about $1 \mathrm{fs}$. During this first femtosecond the hole is mostly transferred to 
the MOs 11 (red line), 13 (green line) and 19 (yellow line). The hole returns to the 16 th $\mathrm{MO}$ within $3 \mathrm{fs}$, when the hole occupation of the 16 th $\mathrm{MO}$ raises to $0.8 \mathrm{e}$. The occupation of the initially ionized $\mathrm{MO} 16$ decreases faster than after the ionization of the 11th $\mathrm{MO}$ as the occupation of the 16 th $\mathrm{MO}$ drops to $0.3 e$ within less than 2 fs.

After 4 fs the hole is mostly transferred to the MOs 13 (green line) and 20 (cyan line), which are located at opposing ends of the molecule. Then (within $8 \mathrm{fs}$ and $12 \mathrm{fs}$ ) the hole oscillates between the MO 19, mostly located on the carboxyl group, and the $\mathrm{MO} 20$, located mostly on the amine group. That means that the hole moves from one end of the molecule, the carboxyl group, to the other end of the molecule, the amine group, within $2 \mathrm{fs}$.

Like in the electron dynamics after ionization of the 11th $\mathrm{MO}$, the $\beta$ MOs show no dynamics.

In both cases, ionization of the 11th $\mathrm{MO}$ and the 16th MO, the TDHF based approach predicts dynamics of the valence MOs on a femtosecond time scale. In both cases the dynamics spreads over the whole molecule. Especially during the dynamics after the ionization of the 16th MO the hole oscillates between the ends of the molecule.

The dynamics observed here seem to best described by the hole diffusing through the valence MOs. This view is supported by the fact that the $\beta$ MOs only show little dynamics during the simulations.

To judge the reliability of these predictions, the results will be compared to the results from Reference [74] in the next section.

\subsubsection{Comparison with Literature Results}

Kuleff and co-workers[73] used a highly correlated ab initio method (non-Dyson ADC(3)[75]) to study the electron dynamics in glycine in a similar setup as discussed above. They interpret their results in terms of the natural charge orbitals and the corresponding hole occupation numbers (cf. Equation (5.4)).

In case of the ionization of the 11th MO the symmetric MOs 11, 13 and 20 and the antisymmetric MOs 17 and 21 are involved in the electron dynamics. The hole essentially oscillates between the MOs 11, 13 and 20. After about $8.3 \mathrm{fs}$ the hole has moved to the $13^{\text {th }} \mathrm{MO}$. The process is then reverted and the hole is back in the initially ionized 11th $\mathrm{MO}$ after about $11.2 \mathrm{fs}$. In this process the hole moves from the carboxyl group through the $C-C$ bond to the opposing end of the molecule. At the same time an oscillation between the $17^{\text {th }}$ and 21 st $\mathrm{MO}$ is induced where a 
"negative" hole is created in the lowest unoccupied molecular orbital (LUMO), i.e. part of an electron charge is transferred into the LUMO.

After the ionization from the 16th MO the hole oscillates between the MOs 16 and 15 with a period of $5 \mathrm{fs}$. The hole is initially distributed over the whole molecule. After about $2 \mathrm{fs}$ the hole is mostly located on the $\mathrm{CO}$ and $\mathrm{OH}$ groups.

The results Kuleff and co-workers[73] do not show the diffusion like behavior observed in this work. The method of Kuleff et al. essentially uses a linear expansion of the cationic states of the molecule. The electron dynamics are determined by the ionized states to which the removal of the electron from the two MOs 11 and 16 contributes to, which likely explains the clear oscillatory dynamics between few MOs.

Both approaches show femtosecond dynamics of the electronic wavefunction after an instantaneous ionization of a MO. However, the dynamics differ between the two approaches. The dynamics predicted by the TDHF approch are in both cases faster than those predicted by the non-Dyson ADC(3) approach.

Despite the mean-field description of the electron interaction in TDHF theory part of the electron correlation is recovered in our explicit time-dependent treatment because the MOs react to the change of the charge density. The explicit time-dependent treatment does not fit into usual hierarchy of configuration interaction (CI) and perturbation treatment of electron correlation in stationary electronic states. The non-Dyson ADC(3), however, describes the electron correlation much more accurate than our approach. Thus, our results are probably less reliable than those obtained by Kuleff and co-workers. 


\subsection{PENNA}

In the previous section it was shown that the TDHF based approach used in this work does not reproduce the charge migration dynamics in glycine predicted by a correlated method. Due to the complex electron dynamics in glycine a comparison of the different results is somewhat cumbersome.

The PENNA was chosen as a second test system because very distinct electron dynamics have been predicted after ionization of the HOMO[74].

The equilibrium structure (see Figure 5.4) of the PENNA molecule was determined using the Gaussian $03[78,83,84]$ program with the $6-31+G^{*}[85-87]$ basis set ${ }^{1}$. The extended structure was obtained by extending the $C_{1}-C_{2}$ bond by $0.2 \AA$

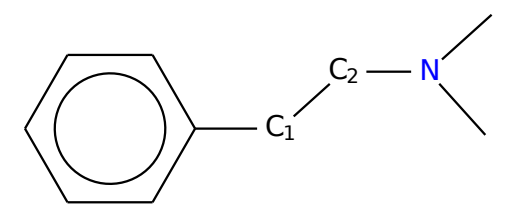

Figure 5.4: Sketch of the PENNA structure.

(for the exact coordinates consider Appendix A.2).

The initial state for the simulation was prepared by instantaneously removing an electron from the HOMO. The HOMO for in both structures located on the benzene ring.

Figure 5.5 shows the time-evolution of the wavefunction after the ionization in both structures (left/solid lines for the equilibrium structure and right/dashed lines for the extended structure). In both cases the initial HOMO (black line), the initial HOMO-1 (green line) and the initial HOMO-2 (red line) are involved in the electron dynamics. The HOMO-1 is also located on the benzene ring like the HOMO. The HOMO-2 is located on the opposing end of the molecule.

In the equilibrium structure simulation the hole occupation of the initially ionized HOMO (black) decreases by about $0.15 e$. About $0.05 e$ of the hole is transferred to the HOMO- 1 (green), which is located on the benzene ring. About $0.1 e$ moves to the other end of the molecule into the HOMO-2 (red). The occupation of the three MOs then oscillates with a period of about $1.5 \mathrm{fs}$.

The electron dynamics in the extended structure are similar to the dynamics in the equilibrium structure. About $0.2 e$ of the hole travels along the molecule into

\footnotetext{
${ }^{1}$ As obtained from the EMSL basis set exchange[81, 82].
} 



Figure 5.5: Time-evolution of the electronic wavefunction after ionization of the highest occupied molecular orbital. The left row of isosurface plots shows the initial molecular orbital of the equilibrium structure. The solid lines indicate the hole occupation of the initial molecular orbitals of the equilibrium structure. Analogously the right row of isosurface plots and the dashed lines correspond to the initial molecular orbitals of the extended structure.

the HOMO-2 (red). That is about twice as much as in the case of the equilibrium structure.

The HOMO and the HOMO-2 differ between the geometries. In the extended structure the HOMO is more located on the nitrogen than in the equilibrium structure. Additionally, in the extended structure the HOMO-2 is partly located on the benzene ring. Thus there is more spatial overlap between these MOs. This contact presumably induces increased coupling, what increases the hole transfer from the benzene to the nitrogen group.

Lünnemann and co-workers[74] used the same correlated method as in Kuleff and co-workers[73] to study the electron in PENNA upon instantaneous ionization of the HOMO. They use a slightly different geometry (the optimized geometry using $\mathrm{BP} 86[88,89], / \mathrm{SV}(\mathrm{P})[90])$. In case of their equilibrium geometry, a partial relocation of the hole from the benzene ring to the nitrogen site within $4 \mathrm{fs}$ is observed. For the extended $C_{1}-C_{2}$ bond not only an enhancement of the hole 
relocation is observed, but almost the complete hole moves to the nitrogen site in about $4 \mathrm{fs}$.

Kuleff et al.[76] applied a single determinant approach to the same problem. They use the (time-independent) Kohn-Sham operator of the cationic state to propagate the wavefunction. In the extended geometry they observe that a part of the hole moves to the nitrogen site of the PENNA molecule. This process takes place in less than $2 \mathrm{fs}$. However, no complete relocation of the hole is observed.

The electron dynamics predicted by the TDHF used in the current work resemble the electron dynamics predicted in Reference[76] in the equilibrium structure. The movement of the hole is, indeed, about 4 times faster. In the case of the elongated $\mathrm{C}_{1}-\mathrm{C}_{2}$ bond, no complete relocation of the hole is observed for both single determinant approaches. Both single determinant approaches yield much faster electron dynamics than the correlated method. 



\section{6 \\ Conclusion \& Outlook}

In the first part of this work we have used time-dependent Hartree-Fock (TDHF) theory to study the electron dynamics in multi-electron systems after both coreand valence-shell ionization.

In Chapter 3 the dynamics in a one-dimensional model system, where the Coulomb interaction was replaced by a soft-core Coulomb potential, were simulated after instantaneous ionization of the core-shell. In both the four- and six-particle systems the core-hole state has enough excess energy to emit another particle from the valence-shell. An emission of a valence particle was, however, not observed in either of the systems. In the six-particle system a transition occurs during the simulation in which the core hole is refilled, but there is distinct emission of a valence particle. Unfortunately, there is no feature in the observed dynamics that could explain the difference between the two systems. Despite these differences our TDHF based approach is capable of describing the decay of a core hole state in the one-dimensional model system.

In Chapter 4 a similar approach was used to simulate the electron dynamics in beryllium after instantaneous core-shell ionization. In addition to the numerical grid already deployed for the model system, a B-spline basis was used to exclude possible limitations introduced by the use of a specific basis set.

Using the numerical grid as a basis set is appealing because the implementation of the working equations with the numerical grid is simple. Despite the more complex implementation, the B-spline basis offers two main advantages over the numerical grid. The use of a non-uniform knot sequence allows a good description of both the bound and the continuum molecular orbitals (MOs). Furthermore, the energies of the continuum MOs can be effectively located below a certain threshold. These properties together allow for a compact B-spline basis, which still describes both the bound and continuum MOs better than a much larger numerical grid. 
With neither basis set an Auger or Auger-like process was observed during the expected core-hole lifetime of beryllium (7.0 fs - $32.9 \mathrm{fs}[63-66])$. It cannot finally be excluded that the lack of the Auger decay in the simulation is caused by limitations of the used basis set. As two different flexible basis sets were used, it seems, however, reasonable to conclude that TDHF theory is not sufficient to describe the Auger decay properly. Especially, it is likely the limited description of electron correlation, due to the mean-field interactions among the electrons, that constitutes that limitation.

Interestingly, the dynamics observed in beryllium resembles the dynamics in the four-particle model system. In both cases both the $\alpha$ and $\beta$ valence orbitals oscillate strongly in space after the ionization. These oscillations are likely to be caused by the perturbation due to the reduced shielding of the central charge.

In Chapter 5, the electron dynamics after instantaneous ionization of a valence $\mathrm{MO}$ were simulated in two small organic molecules. For the molecules gaussian basis sets known from quantum chemistry were employed to make the simulations computationally accessible. The observed dynamics in glycine after valence ionization resemble the created hole charge diffusing through the valence MOs. Electron dynamics obtained with a correlated method by Kuleff and co-workers[73] in a similar setup show, however, a clear oscillation of the hole among few MOs. Similarly, the charge migration process predicted by the same correlated method in 2phenylethyl-N,N-dimethylamine (PENNA)[74] is not reproduced by our TDHF approach. As the charge migration dynamics are driven by electron correlation [23, 24] the TDHF based approach used in the current work lacks the proper description of the electron correlation to simulate the charge migration dynamics.

We initially set out to simulate the electron dynamics during the Auger decay after core-shell photo-ionization to study the impact of these electron dynamics on the early phase of the Coulomb explosion. As our TDHF based approach does not properly describe the Auger decay due to the insufficient description of the electron correlation it cannot be used to simulate the electron dynamics in the early phase of the Coulomb explosion. Thus, one has to move beyond the TDHF description of the electron dynamics.

It may be worthwhile to systematically investigate the electron correlation involved in the Auger decay. To disentangle the effects of electron correlation a linear expansion of the wavefunction in terms of the eigenfunctions of the electronic Hamiltonian should be used. The different levels of electron correlation are implemented by using different methods to determine the electronic Hamiltonian and the respective eigenfunctions. An obvious choice for the excited state method are configuration interaction (CI)[91] methods like configuration interaction singles 
(CIS)[92], configuration interaction singles with perturbation treatment of doubles (CIS(D)) $[93,94]$ and configuration interaction singles and doubles (CISD)[91] as this allows for a systematic enhancement of the inclusion of electron correlation. The determination of the excited states is, however, not limited to CI methods. E.g., Sonk and co-workers successfully used a similar approach employing $\mathrm{CI}$ as well as coupled cluster (CC) methods to simulate the electronic response in butadiene to a short optical laser pulse[95].

Note that, while the determination of e.g. the ground state is much more expensive using CI or CC[96] method compared to Hartree-Fock (HF), this is not necessarily the case for the electron dynamics simulations. In our TDHF based approach the Fock matrix has to be assembled and diagonalized in every timestep. When using a linear expansion of the electronic wavefunction in terms of the eigenfunctions of the Hamiltonian instead the Hamiltonian matrix has to be assembled and diagonalized - at least in principle - only once. With a method that yields compact Hamiltonian matrices like CIS or CIS(D) the electron dynamics may be computationally more efficient than with our current TDHF method.

As a first step, the approach discussed above could be applied to atoms employing the MOs derived from a B-spline HF calculation. To study molecules, a slightly different approach is, however, necessary because a B-spline basis set would not be computationally feasible. There are, indeed, methods to construct continuum MOs in molecules, like the single center expansion $[97,98]$, that could be adapted to be used together with a time-dependent approach. 

Part II

\section{Photo-Absorption Induced Molecular Dynamics}





\section{7 \\ Conical Intersections}

Conical intersections[99-101] form the basis of photo-isomerization reactions as they offer the pathway for the molecular dynamics (MD) mediated decay of electronic excited states in molecules[25, 102]. In contrast to fluorescence, the energy of the absorbed photon is converted into kinetic energy as the system evolves along the excited state potential energy surface (PES) through the conical intersection to the ground state. This kinetic energy then induces a change of the molecular structure leading to photo-isomerization.

In the following, PESs will be introduced because the conical intersections arise from crossings of different PESs. Then, conical intersections will be discussed in terms of the previously derived PESs and the coupling among them.

\subsection{Potential Energy Surfaces}

Potential energy surfaces are a consequence of the approximated solution of the molecular Schrödinger equation

$$
i \frac{\partial}{\partial t} \Psi=\hat{H} \Psi=\left(\hat{T}^{N}+\hat{T}^{e}+\hat{V}\right) \Psi,
$$

where $\hat{T}^{e}$ and $\hat{T}^{N}$ are the electronic and nuclear kinetic energy operators and $\hat{V}$ is the potential operator. To simplify Equation (7.1) the Born-Oppenheimer approximation is invoked[103, 104]. Thereto, adiabatic states[105] of the instantaneous electronic Hamiltonian (2.2)

$$
\hat{H}^{e}(\boldsymbol{r}, \boldsymbol{R}) \Psi_{n}^{e}(\boldsymbol{r} ; \boldsymbol{R})=V_{n}(\boldsymbol{R}) \Psi_{n}^{e}(\boldsymbol{r} ; \boldsymbol{R})
$$


where $\boldsymbol{r}$ are the electronic and $\boldsymbol{R}$ the nuclear coordinates, are used to expand the total wavefunction

$$
\Psi(t, r, \boldsymbol{R})=\sum_{n} \Psi_{n}^{e}(\boldsymbol{r} ; \boldsymbol{R}) \Psi_{n}^{N}(t, \boldsymbol{R}),
$$

which is usually called Born-Oppenheimer expansion[106]. Inserting this expansion into the Schrödinger equation (7.1) and projecting onto another adiabatic state $\Psi_{m}^{e}$ yields the Hamiltonian[107]

$$
\begin{aligned}
\hat{H}_{m n}(\boldsymbol{R}) & =\delta_{m n}\left(\hat{T}^{N}+V_{m}(\boldsymbol{R})\right)-\left\langle\Psi_{m}^{e}\left|\hat{T}^{N}\right| \Psi_{n}^{e}\right\rangle \\
& =\delta_{m n}\left(\hat{T}^{N}+V_{m}(\boldsymbol{R})\right)-\hat{\Lambda}_{m n}(\boldsymbol{R}) .
\end{aligned}
$$

The nonadiabatic coupling $\hat{\Lambda}$ results from the parametric dependence of the adiabatic states on the nuclear coordinates and it couples different adiabatic states. If the adiabatic states are energetically well separated, the nonadiabatic coupling is usually because the nonadiabatic coupling is proportional to the inverse of the energy difference[107]. The strength of the nonadiabatic coupling is used in one of the hopping algorithms, the fewest switches surface hopping (FSSH) algorithm, used in this work as part of the hopping criterion (cf. Section 8.4).

Neglecting the off-diagonal terms of the nonadiabatic coupling renders the Hamiltonian (7.4) diagonal and the wavefunction (7.3) reduces to a single product

$$
\Psi(\boldsymbol{r}, \boldsymbol{R})=\Psi_{n}^{e}(\boldsymbol{r} ; \boldsymbol{R}) \Psi_{n}^{N}(\boldsymbol{R}) .
$$

The single product ansatz for the wavefunction is usually called the Born-Oppenheimer approximation[107]. If the nonadiabatic coupling is neglected entirely, the nuclear wavefunction evolves according to a single PES $V_{n}(\boldsymbol{R})$

$$
i \frac{\partial}{\partial t} \Psi^{N}(t, \boldsymbol{R})=\left(\hat{T}^{N}+V_{n}(\boldsymbol{R})\right) \Psi^{N}(t, \boldsymbol{R}) .
$$

In this work the nuclear Schrödinger equation (7.6) was not solved explicitly, but the time-evolution of the nuclear wavefunction was approximated by a swarm of classical trajectories, which evolve according to the PES $V_{N}(\boldsymbol{R})$ (cf. Section 8.1). 


\subsection{Surface Intersections}

Conical intersections are regions in molecular configuration space where two (or more) PESs are degenerate, i.e.,

$$
V_{1}(\boldsymbol{R})=V_{2}(\boldsymbol{R}) .
$$

Conical intersections play a prominent role in photo-chemical reactions as they allow radiationless decay of excited electronic states via $\operatorname{MD}[25,102]$.

At a conical intersection the off-diagonal elements of the nonadiabatic coupling diverge $[105,107]$ and the Born-Oppenheimer approximation breaks down. To solve the Schrödinger equation (7.1) at a conical intersection, the diabatic electronic states $\boldsymbol{\Phi}^{e}$ are introduced[107] via a unitary transformation from the adiabatic states $(7.2)$

$$
\boldsymbol{\Phi}^{e}=\boldsymbol{U}(\boldsymbol{R}) \mathbf{\Psi}^{e} .
$$

In the diabatic representation the Hamiltonian (7.4) is transformed to

$$
\hat{H}_{m n}(\boldsymbol{R})=\hat{T}_{N} \delta_{m n}+W_{m n}(\boldsymbol{R}),
$$

with the local potential $W_{m n}(\boldsymbol{R})$. This yields the Schrödinger equation in matrix notation

$$
\boldsymbol{H} \boldsymbol{\Psi}^{N}=\left(\hat{T}_{N} \mathbf{1}+\boldsymbol{W}(\boldsymbol{R})\right) \boldsymbol{\Psi}^{N}=\boldsymbol{E} \boldsymbol{\Psi}^{N} .
$$

The diabatic states (7.8) are determined only up to a global phase, that is independent of $\boldsymbol{R}$. With this global phase the diabatic states can be constructed such that they are identical to the adiabatic states at a certain reference geometry $\boldsymbol{R}_{0}$. Expanding the diabatic potential for two diabatic states at $\boldsymbol{R}_{0}$ to first order in the displacements of the nuclei $\Delta \boldsymbol{R}$, yields

$$
\begin{aligned}
\boldsymbol{W}(\boldsymbol{R}) & =\boldsymbol{W}^{(0)}+\boldsymbol{W}^{(1)}+\cdots, \\
\boldsymbol{W}^{(0)} & =\frac{V_{1}+V_{2}}{2} \mathbf{1}+\left(\begin{array}{cc}
-\frac{V_{2}-V_{1}}{2} & 0 \\
0 & \frac{V_{2}-V_{1}}{2}
\end{array}\right)=\boldsymbol{V}\left(\boldsymbol{R}_{0}\right), \\
\boldsymbol{W}^{(1)} & =\left.\frac{1}{2} \nabla_{\boldsymbol{R}}\left(V_{2}+V_{1}\right)\right|_{\boldsymbol{R}_{0}} \boldsymbol{\Delta} \boldsymbol{R} \mathbf{1}+\left(\begin{array}{cc}
-\frac{1}{2} \boldsymbol{h} \cdot \Delta \boldsymbol{R} & \boldsymbol{g} \cdot \boldsymbol{\Delta R} \\
\boldsymbol{g} \cdot \Delta \boldsymbol{R} & \frac{1}{2} \boldsymbol{h} \cdot \Delta \boldsymbol{R}
\end{array}\right),
\end{aligned}
$$


with the derivative coupling

$$
g=\nabla_{\boldsymbol{R}}\left\langle\Psi_{1}^{e}\left|\hat{H}^{e}\right| \Psi_{2}^{e}\right\rangle
$$

and gradient difference vectors

$$
\boldsymbol{h}=\nabla_{\boldsymbol{R}}\left(V_{2}-V_{1}\right)
$$

If the adiabatic states are degenerate at the reference point $\boldsymbol{R}_{0}$, the zeroth order contribution $\boldsymbol{W}^{(0)}$ to the diabatic potential becomes constant and is set to zero. In the vicinity of the conical intersection the adiabatic energies are given by diagonalizing $\boldsymbol{W}^{(1)}$

$$
V_{1,2}(\boldsymbol{\Delta} \boldsymbol{R})=\left.\frac{1}{2} \nabla_{\boldsymbol{R}}\left(E_{2}-E_{1}\right)\right|_{\boldsymbol{R}_{0}} \pm \frac{1}{2} \sqrt{(\boldsymbol{h} \cdot \Delta \boldsymbol{R})^{2}+4(g \cdot \Delta \boldsymbol{R})^{2}} .
$$

Thus, two conditions need to be fulfilled for the electronic states to be degenerate at $\boldsymbol{R}_{0}$

$$
\begin{aligned}
& \boldsymbol{h} \cdot \Delta \boldsymbol{R}=0 \\
& \boldsymbol{g} \cdot \Delta \boldsymbol{R}=0
\end{aligned}
$$

In the space spanned by the gradient difference $\boldsymbol{h}$ and the derivative coupling vector $g$ the degeneracy of the two electronic states is lifted to first order. This space is also called branching space or $\mathrm{g}$-h plane. The space perpendicular to the branching space is usually called intersection or seam space. In this space the degeneracy persists to at least second order[108].

For the classical trajectories employed in this work, special care needs to be taken at conical intersections because the PESs become degenerate and there is no unique force acting on the nuclei. Surface hopping algorithms[29] (cf. Sections 8.3 and 8.4) were used to determine the PES whereon the trajectories evolve. 


\section{8 \\ Methods}

In this chapter the methods necessary to simulate photo-chemical reactions will be discussed.

To determine the nuclear dynamics it is necessary to solve equation (7.6). This can be done quantum-mechanically using wavepacket dynamics with precalculated potential energy surface (PES). This approach, however, poses a serious computational challenge and limits its applicability to systems with only few nuclear degrees of freedom (DOF)[28]. So we approximate the time-evolution of the nuclear wavefunction by classical trajectories. These trajectories are then obtained by solving the Newton's equation of motion given the by PES $V(\boldsymbol{R})$ of a given electronic state

$$
M \ddot{R}=-\nabla_{\boldsymbol{R}} V(\boldsymbol{R})
$$

(cf. Section 8.1).

We determined PES "on the fly" by solving the electronic Schrödinger equation (2.2) for the current molecular geometry. This approach is also called ab initio molecular dynamics (AIMD)[109]. The AIMD approach is limited by the computational costs of solving the electronic Schrödinger equation (2.2). Depending on the model used, the system size is limited to hundreds (density functional theory (DFT)[110]) to a dozen (highly accurate correlated post-Hartree-Fock methods like coupled cluster (CC)[96]) of atoms.

Instead of explicitly solving the electronic Schrödinger equation it is also possible to parametrize the PES $V(\boldsymbol{R})$ (usually of the electronic ground state) using a force field (FF) $V_{M M}(\boldsymbol{R})$ (cf. Section 8.1.1). Molecular dynamics (MD) can successfully be applied to large proteins or protein complexes (up to 1o million atoms) reaching timescales up to a millisecond[111].

On the one hand, an ab initio description of the involved electronic states is necessary. On the other hand, the influence of the surrounding protein and/or 
solvent environment needs to be taken into account. To meet both requirements a combination of AIMD and MD is used. The explicit quantum mechanical description of the PES is restricted to a certain region of interest, where the photochemical reaction takes place (i.e. the chromophore). The rest of the system is then described by classical MD. These two regions are then combined in a quantum mechanics/molecular mechanics (QM/MM) approach (cf. Section 8.1.2)[112-114].

To simulate photo-chemical processes, a reliable description of almost degenerate electronic states in the vicinity of a conical intersection is needed. To this end, we use the complete active space self-consistent field (CASSCF)[115] (cf. Section 8.2) method to solve the electronic Schrödinger equation (2.2). The CASSCF method is a variant of the multi-configurational self-consistent field (MCSCF) method $[116,117]$, which combines Hartree-Fock (HF) (c.f. 2.1) and configuration interaction $(\mathrm{CI})[91]$. Instead of using a predetermined set of molecular orbital (MO) to solve the CI problem, the MO and CI coefficients are optimized in parallel. This allows a balanced description of multiple electronic states as most of the static correlation is accounted for. The MCSCF method also allows the calculation of the nonadiabatic coupling[118] (c.f. Chapter 7), which are needed for certain surface hopping algorithms.

When a trajectory of an MD simulation, reaches regions in configuration space with strong nonadiabatic coupling or even a conical intersection, the Born-Oppenheimer approximation breaks down. Especially the approximation of a classical trajectory evolving on a single PES is not valid anymore. We employed different surface hopping algorithms, the diabatic surface hopping (DSH)[36] and fewest switches surface hopping (FSSH) [119, 120] algorithms, to determine, when the trajectory switches from one PES to another.

Both surface hopping algorithms were implemented as QM/MM interfaces in the MD package Gromacs[121-125]. The necessary CASSCF calculations were performed using the quantum chemistry program Molpro 2009.1[126-135]. 


\subsection{Molecular Dynamics}

For an MD simulation, the Newton's equation of motions (eq. (8.1)) of the classical nuclei

$$
\begin{aligned}
\dot{\boldsymbol{R}}(t) & =\boldsymbol{V}(t) \\
\dot{\boldsymbol{V}}(t) & =-\frac{1}{M} \nabla_{\boldsymbol{R}} V(\boldsymbol{R}(t))
\end{aligned}
$$

are solved numerically to generate a (quasi) continuous trajectory of the molecular geometry $\boldsymbol{R}(t)$. We used the second order leapfrog[136] algorithms, as available in Gromacs, to solve (8.2) numerically.

To generate other than the NVE, fixed number of particles, fixed volume and fixed total energy, ensemble, the temperature[137, 138] and pressure[137] coupling algorithms available in Gromacs were used. To resemble experimental condition the NpT ensemble was employed in the non-vacuum simulations (cf. Section 9.2 and Chapter 10).

\subsubsection{Force Fields}

An FF $V_{M M}(\boldsymbol{R})$ is an parametrization of the PES $V(\boldsymbol{R})$ of (usually) the electronic ground state of a molecule. Therefore, the PES is decomposed into different contributions[139]

$$
\begin{aligned}
V_{M M} & =\sum_{(A, B)}^{N_{\text {bonds }}} \frac{1}{2} k_{A B}^{b}\left(\boldsymbol{R}_{A B}-\boldsymbol{R}_{A B}^{0}\right)^{2} \\
& +\sum_{(A, B, C)}^{N_{\text {angles }}} \frac{1}{2} k_{A B C}^{\alpha}\left(\alpha_{A B C}-\alpha_{A B C}^{0}\right)^{2} \\
& +\sum_{(A, B, C, D)}^{N_{\text {dihedrals }}} k_{A B C D}^{\theta}\left(1+\cos \left(n \theta_{A B C D}-\theta_{A B C D}^{0}\right)\right) \\
& +\sum_{(A, B, C, D)}^{N_{\text {improper }}} \frac{1}{2} k_{A B C D}^{\xi}\left(\xi_{A B C D}-\xi_{A B C D}^{0}\right)^{2} \\
& +\sum_{(A, B)}^{N_{\text {pairs }}} \frac{C_{A B}^{12}}{\left|\boldsymbol{R}_{A B}\right|^{12}}-\frac{C_{A B}^{6}}{\left|\boldsymbol{R}_{A B}\right|^{6}}
\end{aligned}
$$




$$
+\sum_{(A, B)}^{N_{\text {pairs }}} \frac{\delta_{A} \delta_{B}}{4 \pi \varepsilon_{0} \varepsilon_{r}\left|\boldsymbol{R}_{A B}\right|} .
$$

The first four terms describe interactions among bonded atoms. The angular potentials act among three subsequent bonded atoms and likewise the dihedral potential among four subsequent bonded atoms. The improper dihedral potential is imposed on out of plane angles. The Lennard-Jones potential descibes the vander-Waals interactions. The Coulomb potential acts among the partial charges of all atoms. The parameters in (8.3) are either determined by fitting experimental data, ab initio calculations or both[140-142].

In our simulations, the FFs were used to describe the molecular mechanics (MM) region of the system, i.e. the protein environment and the solvent.

\subsubsection{QM/MM}

We used the electrostatic embedding scheme (see Figure 8.1.1) to combine the quantum mechanics (QM) description of the chromophore and the MM description of the rest of the system[36, 112].

The interaction among atoms in the MM region is given by an FF (c.f. section 8.1.1). In the QM region the electronic Schrödinger equation (2.2) is solved explicitly to determine the forces on the QM atoms. The partial charges of the MM atoms are taken into account in the QM calculation polarising the electronic wavefunction. In turn the electronic wavefunction exerts additional long range forces onto those MM charges. The van-der-Vaals interaction between QM and MM atoms is described at the FF level.

If a bond is cleaved in the partitioning, it is capped with a monovalent link atom like hydrogen in the QM calculation. The QM force acting on this hydrogen is distributed evenly among the two originally bonded atoms.

\subsection{Excited State Quantum Chemistry}

There are two main approaches to the calculation of excited states. Due to the linear parametrization of the wavefunction used in $\mathrm{CI}[91]$ methods, the calculation of excited state is in principle straightforward. The excited states and the excited state energies are the corresponding eigenvectors and eigenvalues of the Hamiltonian matrix. Other excited state methods are based on the (linear) response 


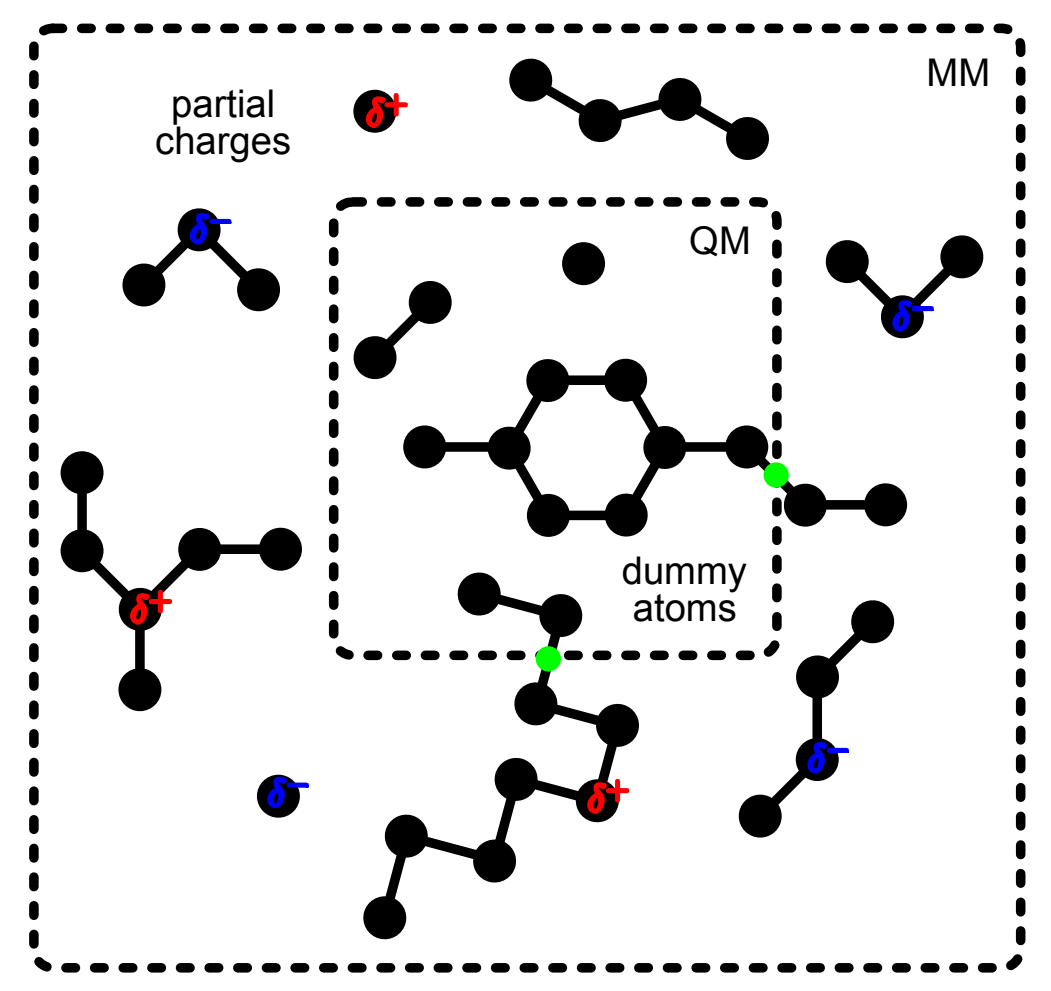

Figure 8.1: Sketch of the mechanical embedding scheme.

approach. To calculate excited states, the perturbation is taken to be an oscillating electric field. The excited states and excitation energies can then be obtained from the corresponding poles and residues of the linear response function[143]. Timedependent density functional theory (TDDFT)[17] and equation-of-motion coupled cluster (EOM-CC) theory[96] are based on linear response theories.

For the simulation of photo-chemical processes a method is required that is capable of describing the (quasi-) degenerate electronic states in the vicinity of a conical intersection. Single reference methods like TDDFT and EOM-CC are known to perform poorly in such multi-configurational configurations and a multi-reference method is needed[8o]. We deployed the CASSCF method, which is a variant of the MCSCF approach.

In the MCSCF method, the CI ansatz is used

$$
|\Psi\rangle=\sum_{I} c_{I}|I\rangle \text { with } \sum_{I}\left(c_{I}\right)^{2}=1
$$


where the CI expansion is with respect to a set of Slater determinants $\{|I\rangle\}_{I}$. The Slater determinants are defined with respect to a set of orthonormal orbitals $\left\{\chi_{p}\right\}$ (which are not necessarily the HF canonical orbitals). In an MCSCF calculation both the CI coefficients and the MO are optimized. Thus, a converged MCSCF wavefunction is variational.

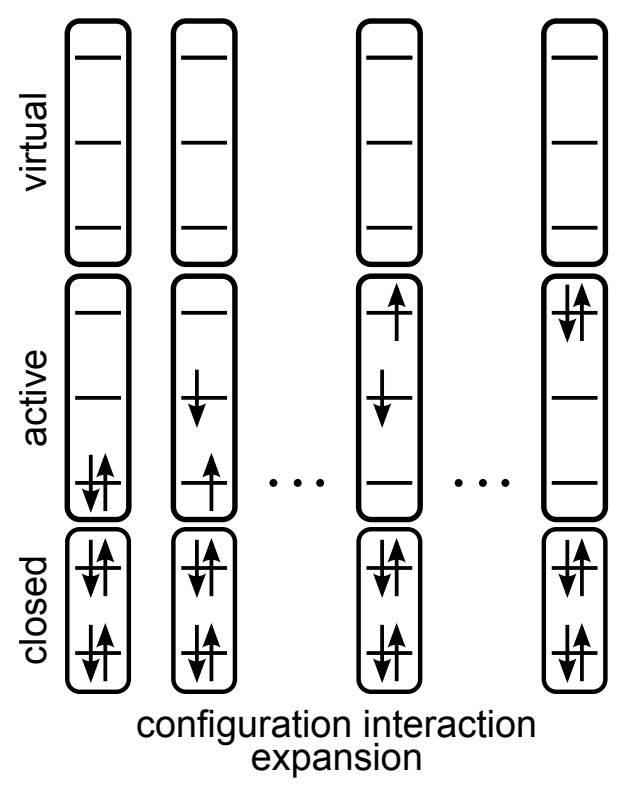

Figure 8.2: The complete active space decomposition of the molecular orbital space. The arrows indicate up or down spin electrons.

To obtain multiple electronic states, i.e. to perform excited state calculation, state averaging is used to avoid root flipping[144, 145]. In state averaging the average energy $E_{\text {average }}$

$$
E_{\text {average }}=\sum_{n} \omega_{n} E_{n}
$$

with the state weights $\omega_{n}$ is minimized. For this purpose, all electronic states are defined with respect to a common set of state averaged MOs. Additionally, the common set of MOs allows easier calculation of off-diagonal elements of electronic operators

$$
O_{m n}=\left\langle\Psi_{m}^{e}|\hat{O}| \Psi_{n}^{e}\right\rangle
$$


Due to the common set of MOs, the state specific energies $E_{n}$ are not fully variational.

The CASSCF method now defines a scheme to select the Slater determinants used in the CI expansion (8.4) (c.f. Figure 8.2). The orbital space is split in three parts. The first set (closed) of orbitals is kept doubly occupied during the calculation and the second set (virtual) is kept empty. The active space is composed from both orbitals, which are occupied and virtual in the original MO set. Within the active space a full configuration interaction (FCI) expansion is made, that is all possible excited Slater determinants contribute to the CI expansion.

\subsection{Diabatic Surface Hopping}

In the DSH algorithms[36] hops from one PES to another only occur when the trajectory crosses the seam of a conical intersection (cf. Section 7.2). Neglecting any nonadiabatic coupling other than the conical intersection itself, is only a valid approximation as long as the molecule does not have extended regions of nonadiabatic coupling. The validity of this approximation, thus, depends on the system under consideration (cf. the discussions in Section 9.3 as well as in Chapter 10).

In the adiabatic basis $\Psi_{n}^{e}$ crossing the seam of a conical intersection is indicated by[146]

$$
\begin{aligned}
S_{m n} & =\left\langle\Psi_{m}^{e}(t) \mid \Psi_{n}^{e}(t+\Delta t)\right\rangle, \\
\left|S_{m m}\right| & \approx\left|S_{n n}\right| \approx 0, \\
\left|S_{m n}\right| & \approx 1,
\end{aligned}
$$

where the electronic wavefunctions of two subsequent MD steps are compared.

For the implementation it is assumed that the orbitals of two subsequent timesteps are sufficiently similar so that (8.7) can be approximated by the scalar product of the $\mathrm{CI}$ vectors

$$
S_{m n} \approx \mathbf{c}_{m} \cdot \mathbf{c}_{n} .
$$

A hop between the adiabatic states $\Psi_{j}^{e}$ and $\Psi_{k}^{e}$ is performed if the energy gap between the states is smaller than 0.1 Hartree and

$$
\left|S_{m m}\right|<0.5 \wedge\left|S_{n n}\right|<0.5 \wedge\left|S_{m n}\right|>0.5 .
$$


The DSH algorithm was used in all three computational studies presented in the following (cf. Sections 9.1 and 9.2 and Chapter 10).

\subsection{Fewest Switches Surface Hopping}

The electronic wavefunction is expanded in the space of adiabatic states

$$
\Psi(t, \mathbf{r}, \mathbf{R})=\sum_{n} c_{n}(t) \Psi_{n}(\mathbf{r}, \mathbf{R}) .
$$

Inserting the ansatz (8.10) into the Schrödinger equation (7.1), projecting onto the adiabatic state $\Psi_{m}^{e}$ and integrating over the electronic degrees of freedom, yields

$$
i \dot{c}_{m}=\sum_{n} c_{n}\left(\delta_{m n} E_{m}-i \dot{\boldsymbol{R}} \cdot \boldsymbol{d}_{m n}\right)=\sum_{n} A_{m n} c_{n}
$$

with the nonadiabatic coupling matrix element $\boldsymbol{d}_{m n}$ (cf. Section 7.2)

$$
\boldsymbol{d}_{m n}=\left\langle\Psi_{m}^{e} \mid \nabla_{\boldsymbol{R}} \Psi_{n}^{e}\right\rangle
$$

Tully proposed the fewest switches surface hopping algorithm[147], where the hopping probability from state $m$ to state $n$ in the time interval $\Delta t$ is given by

$$
P_{m \rightarrow n}=\max \left(\frac{\Delta t b_{n m}}{c_{m} c_{m}^{*}}, 0\right)
$$

with

$$
b_{m n}=-2 \Re\left(c_{m}^{*} c_{n} \dot{\mathbf{R}} \cdot \mathbf{d}_{m n}\right) .
$$

We implemented the time-evolution of the coefficient (8.11) by using the time evolution operator

$$
\mathbf{c}(t+\Delta t)=\exp (-i \mathbf{A} \Delta t) \mathbf{c}(t) \text {. }
$$

The time evolution operator is determined by diagonalizing the matrix $\mathbf{A}$. The diagonalization is always possible as $\dot{\boldsymbol{R}} \cdot \boldsymbol{d}_{m n}$ is skew-symmetric and, thus, $-i \dot{\boldsymbol{R}}$. $\boldsymbol{d}_{m n}$ is hermitian.

The Equation (8.11) is often solved using a integrator like the fourth-order RungeKutta instead[148]. Integrating Equation (8.11) directly probably has performance 
advantages when a large number of adiabatic states is used in the wavefunction expansion 8.10, e.g. when using semi-empirical methods[148]. Because the matrix diagonalizing and thus the evaluation of 8.15 becomes expensive. Here, however, the use of CASSCF is aimed at. Therefore, only a few states were included in the wavefunction expansion 8.10 and the computational costs were dominated by the CASSCF calculation. Hence, there was no need to optimize the propagation of the electronic wavefunction and the propagation scheme described above was chosen out of convenience.

With the new coefficients $c(t+\Delta t)$ the transition probabilities are calculated (equation (8.15)). A random number $\zeta \in[0,1]$ is drawn and a hop from state $m$ to state $n$ is performed if

$$
\sum_{k}^{n-1} P_{m \rightarrow k}<\zeta<\sum_{k}^{n} P_{m \rightarrow k} .
$$

To ensure energy conservation for each trajectory the velocities of the atoms are scaled along the nonadiabatic coupling vector (8.12) in case a hop occurs

$$
\dot{\mathbf{R}}^{\text {new }}=\dot{\mathbf{R}}^{\text {old }}+f \hat{\mathbf{n}},
$$

where $\hat{\mathbf{n}}$ is a unity vector parallel to $\mathbf{d}_{m n}$. The scaling factor $f$ is determined from

$$
\begin{aligned}
E_{\text {kin }}^{\prime \text { new }} & =E_{\text {kin }}^{\prime \text { old }}+\Delta E_{m n} \\
\Rightarrow 0 & =\frac{1}{2} f^{2} \sum_{A} m_{A}+f \sum_{A} m_{A} \dot{\mathrm{R}}_{A} \cdot \hat{\mathrm{n}}_{A}+\Delta E_{m n} .
\end{aligned}
$$

The solution with the smaller modulus was used because it perturbs the system less. In the implementation the vector $\mathbf{R}$ contained both the coordinates of the QM atoms and the point charges taken into account in the QM calculation.

The FSSH algorithm can induce hops at large energy gaps (cf. Section 9.2) what poses two problems. First, a hop at a large energy gap deposits a large amount of kinetic energy into the system. Second, with photo-chemical applications in mind, the hops at large energy gaps take place far away from the seam of the conical intersection. Thus, trajectories encountering hops at large energy gaps usually yield little information about the photo-isomerization pathway.

The hops at large energy gaps are mostly caused by the algorithm constantly building up density in the unoccupied states due to small nonadiabatic coupling. The decoherence correction (DC) scheme was proposed to remedy this problem[120]. 
The density in the unoccupied states is made to decay exponentially. In every step the following correction is made to the expansion coefficients

$$
\begin{aligned}
c_{m}^{\prime} & =c_{m} \exp \left(-\Delta t / \tau_{m n}\right) \forall m \neq n, \\
c_{n}^{\prime} & =c_{n}\left(\frac{1-\sum_{m \neq n}\left|c_{m}^{\prime}\right|^{2}}{\left|c_{n}^{\prime}\right|^{2}}\right)^{\frac{1}{2}}, \\
\tau_{m n} & =\frac{1}{\left|V_{m}-V_{n}\right|}\left(1+\frac{\alpha}{E_{\mathrm{kin}}}\right) .
\end{aligned}
$$

where $n$ is the current state. The correction of the coefficient of the current state $c_{n}$ ensures the overall normalization of the wavefunction. We used a decay constant $\alpha$ of 0.1 Hartree[149].

The FSSH algorithm was used in all three computational studies presented in the following (cf. Sections 9.1 and 9.2 and Chapter 10) while the DC was only employed in the first two. 


\section{9 \\ Applications}

Photo-isomerization is a common process in photo-chemistry. Photo-isomerization is, for example, the first step in a cascade of processes that lead to vision[27]. It is caused by photo-absorption that promotes the system to an excited electronic state. The sudden change of the potential energy surface (PES) due to the switch to a different electronic state triggers a rotation around a bond changing the molecular structure. This change of the molecular structure of the chromophore then induces a cascade of processes that lead to the specific biological function[26, 27].

Protonated Schiff base (PSB) retinals are involved in different light driven biological processes like vision or opening of membrane channels[150, 151]. In their protein environment the PSB retinals undergo a photo-isomerization upon photoabsorption. This structural change in the retinal then triggers the subsequent processes.

We have chosen the protonated Schiff base with two double bonds (PSB2) $\left(C_{1}-N_{2}=C_{3}-C_{4}=C_{5}-C_{6}\right)$ as a test system to compare possible systematic deviations in the predicted photo-isomerization pathways and products among the diabatic surface hopping (DSH) and the fewest switches surface hopping (FSSH) surface hopping algorithms. The PSB2 lends itself to systematic computational studies as it is small in terms of molecular size, and a small complete active space (CAS) suffices for a proper description of the ground and first excited state involved in the photo-isomerization.

The photoactive yellow protein (PYP) is found in salt tolerant Halorhodospira halophila bacteria[30]. The protein is believed to be involved in a light sensing mechanism that allows the bacteria to escape harmful ultraviolet radiation[30]. Its photo-isomerization has been studied both experimentally[31-35] as well as computationally[36-39]. The aim is to validate the isomerization pathway predicted previously with excited state molecular dynamics (MD) using larger active spaces and different hopping algorithms. 


\subsection{Protonated Schiff Base}

The PSB2 (see Figure 9.1) was chosen as an example to compare the different hopping algorithms discussed in Sections 8.3 and 8.4. To this end, the excited state MD of PSB2 were simulated in vacuum using the different hopping algorithms, and the reaction mechanisms and products predicted by the different algorithms were compared.



Figure 9.1: The molecular orbitals composing the active space are shown. The two orbitals in the lower row are mainly occupied in the ground state, whereas the two upper orbitals are occupied in the excited state.

Forty nine initial configurations were generated from taking snapshots every $1 \mathrm{ps}$ from a complete active space self-consistent field $(\mathrm{CASSCF})(4,4) / 3-21 \mathrm{G}^{1}$ ground state trajectory at $T=300 \mathrm{~K}$ using the stochastic dynamics integrator available in Gromacs[152] with the temperature coupling constant $\tau_{t}=0.01 \mathrm{ps}$. For each initial configuration, a trajectory was run with each hopping algorithm (DSH, FSSH and FSSH with decoherence correction (DC) (cf. Sections 8.3 and 8.4). The same seeds were used for the random number generation for the FSSH and FSSH with DC trajectories started form the same initial configuration. One of the DSH trajectories encountered a configuration that the CASSCF calculation does not converge for. This trajectory was excluded from the analysis.

As the excited state of interest is a $\left(\pi, \pi^{*}\right)^{2}$ state, the active state was composed by the four $\pi$ orbitals shown in Figure 9.1.

\footnotetext{
${ }^{1}$ Here the Gaussian notation is used to denote the active space. CAS $(e, o)$ denotes a total $e$ electrons distributed in $o$ orbitals per spin.

${ }^{2}$ The notation $\left(\pi, \pi^{*}\right)$ indicates, that an electron is promoted from a $\pi$ occupied molecular orbital (MO) to $\pi$ virtual $\mathrm{MO}$.
} 
Both the ground state trajectory, which the initial configurations were extracted from, and the orbitals defining the active space were kindly provided by Lela Vuković ${ }^{1}$.

In the following section the excited state dynamics, especially the isomerization pathways and products, are discussed to lay the ground for the comparison of the different hopping algorithms. In Section 9.1.2 the results predicted by the different hopping algorithms are compared.

\subsubsection{Excited State Dynamics}

The dynamics after the vertical excitation follow a common pathway[153]. Figure 9.2 shows the $\mathrm{N}_{2}-\mathrm{C}_{3}, \mathrm{C}_{3}-\mathrm{C}_{4}$ and $\mathrm{C}_{4}-\mathrm{C}_{5}$ bond lengths and the $\mathrm{CN}_{2} \mathrm{C}_{3} \mathrm{C}$ and $\mathrm{CC}_{4} \mathrm{C}_{5} \mathrm{C}$ dihedral angles of one of the trajectories run with the DSH algorithms. First the $\mathrm{N}_{2}-\mathrm{C}_{3}$ and the $\mathrm{C}_{4}-\mathrm{C}_{5}$ extend while the $\mathrm{C}_{3}-\mathrm{C}_{4}$ bond shortens. The

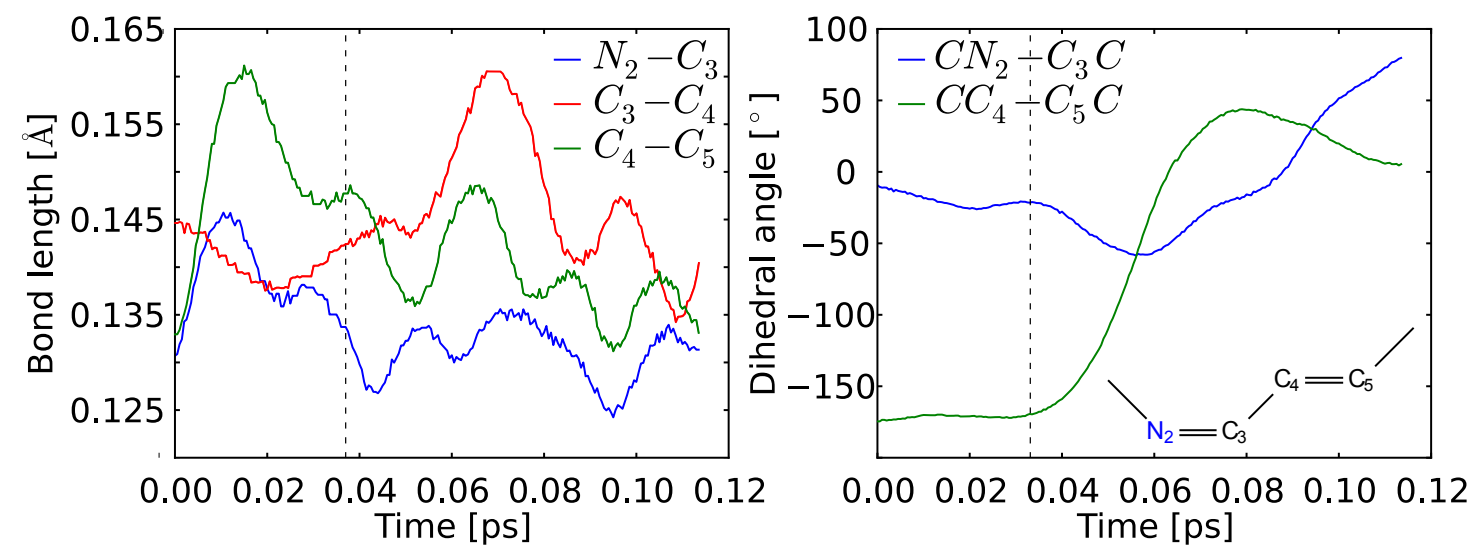

Figure 9.2: Excited state dynamics after vertical excitation. The trajectory shown is one of the diabatic surface hopping trajectories. The vertical dashed line indicates the hop from the excited to the ground state.

change in the bond lengths is accompanied by a bond order inversion of all three bonds. The $\mathrm{N}_{2}-\mathrm{C}_{3}$ and the $\mathrm{C}_{4}-\mathrm{C}_{5}$ bonds change from double to single bond character. The $\mathrm{C}_{3}-\mathrm{C}_{4}$ bond vice versa changes from single to double bond character. Then the system hits the conical intersection (indicated by the dashed line). Afterwards, the system isomerizes. In this particular case, the $\mathrm{CC}_{4} \mathrm{C}_{5} \mathrm{C}$ dihedral angle isomerizes from the trans- to the cis-configuration.

\footnotetext{
${ }^{1}$ Department of Chemistry, University Illinois at Chicago
} 
To compare the excited state dynamics predicted by the different hopping algorithms, the $\mathrm{N}_{2}-\mathrm{C}_{3}$ and the $\mathrm{C}_{4}-\mathrm{C}_{5}$ bond lengths, the $\mathrm{CN}_{2} \mathrm{C}_{3} \mathrm{C}$ and $\mathrm{CC}_{4} \mathrm{C}_{5} \mathrm{C}$ dihedral angles and the products of the isomerization were used.

\subsubsection{Comparison of Hopping Algorithms}

To compare the different hopping algorithms, the important parameters of the excited state dynamics (cf. Section 9.1.1), the $\mathrm{N}_{2}-\mathrm{C}_{3}$ and $\mathrm{C}_{4}-\mathrm{C}_{5}$ bonds and the $\mathrm{CN}_{2} \mathrm{C}_{3} \mathrm{C}$ and $\mathrm{CC}_{4} \mathrm{C}_{5} \mathrm{C}$ dihedral angles, were considered. Furthermore, the excited state lifetimes and the results of the isomerizations were taken into account. Figure

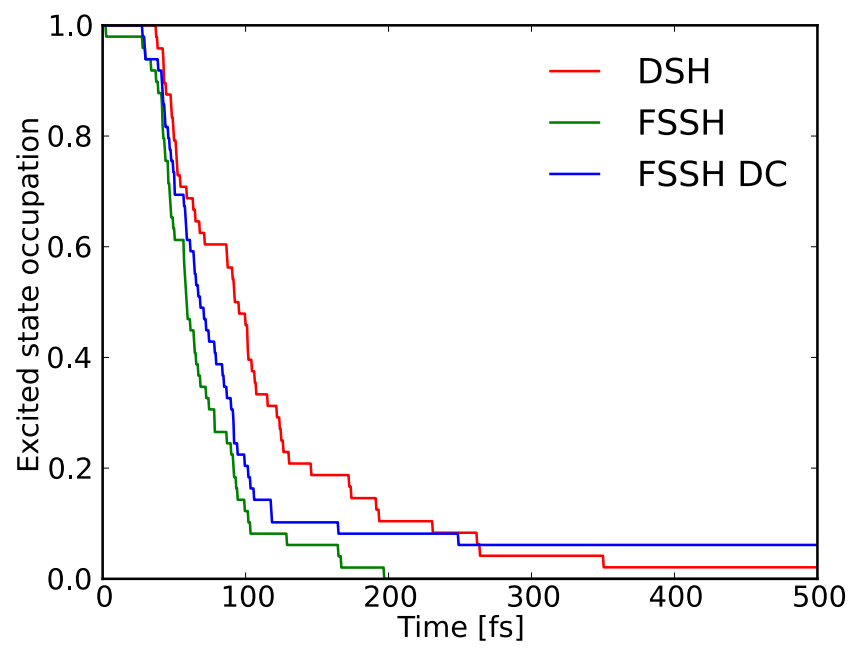

Figure 9.3: The relative occupation of the excited state for the different hopping algorithms is shown. For convenience only the time up to $500 \mathrm{fs}$ is shown.

9.3 shows the occupation of the excited state for the different hopping algorithms. These curves were used to derive the expectation value of the excited state lifetime $\tau$ from

$$
\tau=\frac{\int_{0}^{t_{\operatorname{Max}}} \mathrm{d} t \cdot t \cdot o(t)}{\int_{0}^{t_{\mathrm{Max}}} \mathrm{d} t \cdot o(t)},
$$

where $o(t)$ is the occupation of the excited state. The expected lifetimes and their standard deviations obtained with the different hopping algorithms are summarized in Table 9.1. The curves shown in Figure 9.3 indicate a faster decline of the 


\begin{tabular}{ccc}
\hline DSH & FSSH & FSSH DC \\
\hline $93(97)$ & $44(36)$ & $244(326)$ \\
\hline
\end{tabular}

Table 9.1: Expectation values and standard deviations of the excited state lifetime.

excited state for the two FSSH algorithms before about $200 \mathrm{fs}$. This trend is not reflected in the expected lifetimes. The FSSH DC trajectories have the largest lifetime due to some long trajectories (one trajectory takes about $1.3 \mathrm{ps}$ to reach the ground state). The standard deviation is large as well. Hence, the data does show a significant difference in the excited state lifetimes.

Figure 9.4 shows the distribution of $\mathrm{N}_{2}-\mathrm{C}_{3}$ and $\mathrm{C}_{4}-\mathrm{C}_{5}$ bonds and the $\mathrm{CN}_{2} \mathrm{C}_{3} \mathrm{C}$ and $\mathrm{CC}_{4} \mathrm{C}_{5} \mathrm{C}$ dihedral angles at the hop. None of the two distributions show a clear distinction among the three hopping algorithms. Table 9.2 summarizes the

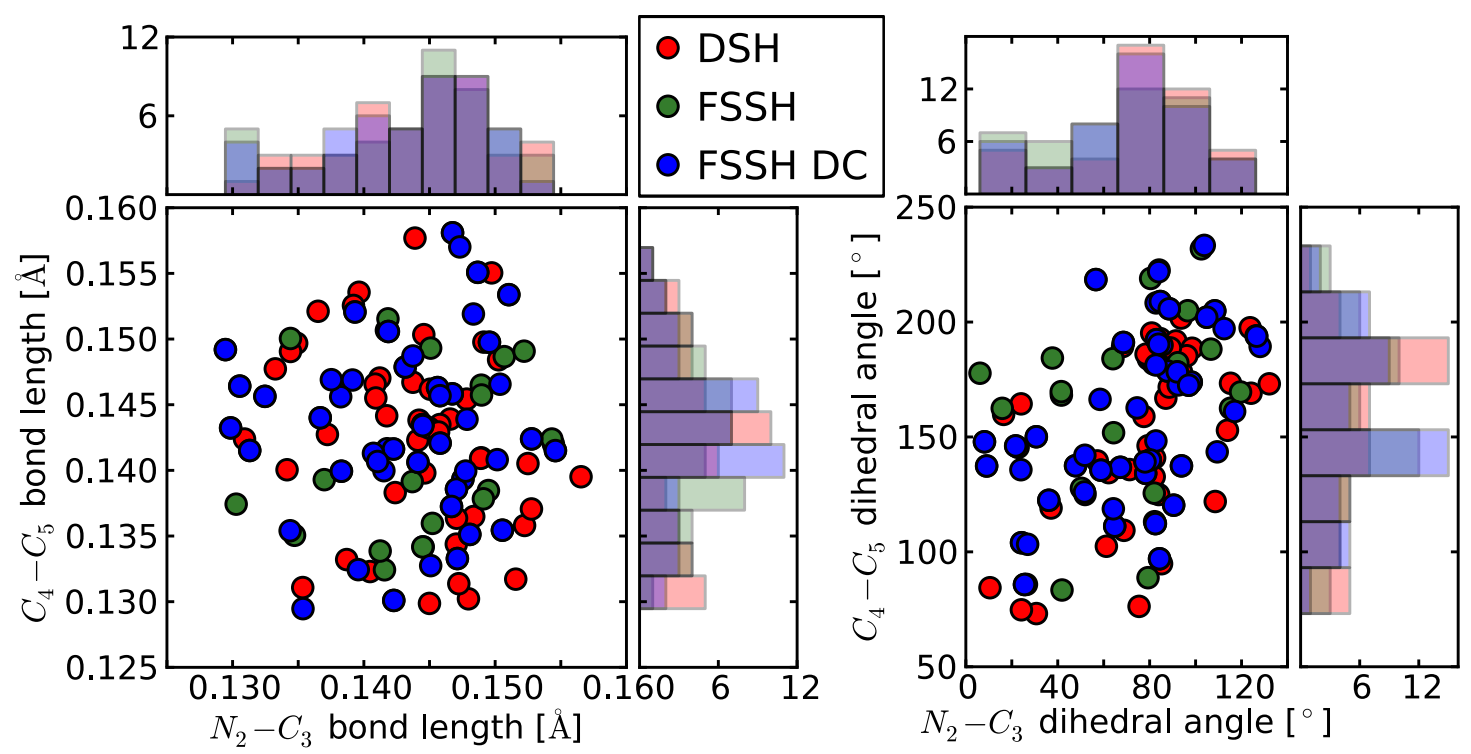

Figure 9.4: Distribution of bond lengths (left) and dihedral angels (right) at the hopping point.

isomerization results for all three hopping algorithms. A bond is considered to isomerize if it rotates more than $70^{\circ}$ and then either rotates back to its initial value or rotates further to more than $150^{\circ}$ from its initial value. To judge the signifi- 


\begin{tabular}{ccccc}
\hline & & DSH & FSSH & FSSH with DC \\
\hline & $N_{\text {traj }}$ & 48 & 49 & 49 \\
$\mathrm{CN}_{2} \mathrm{C}_{3} \mathrm{C}$ & $N_{\text {cis }}$ & 25 & 28 & 29 \\
& $N_{\text {trans }}$ & 12 & 15 & 12 \\
$\mathrm{CC}_{4} \mathrm{C}_{5} \mathrm{C}$ & $N_{\text {cis }}$ & 5 & 5 & 6 \\
& $N_{\text {trans }}$ & 13 & 18 & 17 \\
\hline
\end{tabular}

Table 9.2: The products of the photo-isomerization for the different hopping algorithms. Note that the numbers of the individual isomerizations do not add up to the total number of trajectories as in some cases no isomerization takes place or both bonds isomerize.

cance of the differences in the isomerization results among the different hopping algorithms, I performed a bootstrapping to generate distributions of each number of isomerization products. For better comparability also for the DSH algorithm samples of 49 trajectories were generated. Table 9.3 summarizes the bootstrapping

\begin{tabular}{ccccc}
\hline & & DSH & FSSH & FSSH with DC \\
\hline & $N_{\text {traj }}$ & 49 & 49 & 49 \\
$\mathrm{CN}_{2} \mathrm{C}_{3} \mathrm{C}$ & $N_{\text {cis }}$ & $26(3.5)$ & $28(3.5)$ & $29(3.5)$ \\
& $N_{\text {trans }}$ & $12(3)$ & $15(3.2)$ & $12(3)$ \\
$\mathrm{CC}_{4} \mathrm{C}_{5} \mathrm{C}$ & $N_{\text {cis }}$ & $4(2.1)$ & $5(2.1)$ & $6(2.3)$ \\
& $N_{\text {trans }}$ & $14(3.2)$ & $18(3.4)$ & $17(3.3)$ \\
\hline
\end{tabular}

Table 9.3: Bootstrapped results of the photo-isomerization. The table summarizes the mean values and standard deviations derived from 100,000 samples of 49 trajectories each.

results obtained from 100,000 samples each. The distributions of the number of the isomerization products derived from the bootstrapped were used to calculate the $\mathrm{p}$-values of the observed number of isomerizations for the different hopping algorithms. Table 9.4 summarizes the p-values. For each isomerization product only the p-values with respect to the distribution, that is most different from the other two, is shown. Most of the p-values are larger than 0.2. Thus, there is no significant difference in the predicted numbers of $\mathrm{N}_{2} \mathrm{C}_{3}$ cis, $\mathrm{N}_{2} \mathrm{C}_{3}$ trans and $\mathrm{C}_{4} \mathrm{C}_{5}$ cis isomerizations among the three hopping algorithms. Comparing the number 


\begin{tabular}{cccc}
\hline & DSH & FSSH & FSSH DC \\
\hline $\mathrm{N}_{2} \mathrm{C}_{3}$ cis & ref & 0.33 & 0.24 \\
$\mathrm{~N}_{2} \mathrm{C}_{3}$ trans & 0.22 & ref & 0.22 \\
$\mathrm{C}_{4} \mathrm{C}_{5}$ cis & 0.27 & 0.44 & ref \\
$\mathrm{C}_{4} \mathrm{C}_{5}$ trans & ref & 0.07 & 0.04 \\
\hline
\end{tabular}

Table 9.4: The p-values derived from the bootstrapped distributions for the observed number of each isomerization product are shown. For brevity only the $\mathrm{p}$-values with respect to the distribution, that is most different from the other two, are shown (indicated by "ref") .

of $\mathrm{C}_{4} \mathrm{C}_{5}$ trans isomerizations obtained with the $\mathrm{FSSH}$ algorithms to distribution of $\mathrm{C}_{4} \mathrm{C}_{5}$ trans isomerization derived from the DSH results (last line in Table 9.4) yields the lower p-values of 0.07 and o.04. Hence, the DSH seems to predict less $\mathrm{C}_{4} \mathrm{C}_{5}$ trans isomerizations than the FSSH algorithms. There is, however, no indication for a reason of this deviation.

Overall, there is only a small difference in the reaction products between the hopping algorithms. This similarity is likely to be caused by the specific excited state dynamics of PSB2. The excited state dynamics of PSB2 are governed by a "down hill" process[153]. After the vertical excitation, the molecule drops into the conical intersection within a couple of hundred femtoseconds. There does not seem to be any region of nonadiabatic coupling except for the conical intersection itself, which would lead to different dynamics in the case of the FSSH algorithms. 


\subsection{Photoactive Yellow Protein}

The PYP ${ }^{1}$ contains a chromophore, the deprotonated 4-hydroxy-cinnamic acid ( $\mathrm{p}$ coumaric acid (PCA)), which is covalently linked to the $\gamma$-sulfur of the Cysteine (Cys)69 (see Figure 9.5). The photocycle of the PCA chromophore is sketched

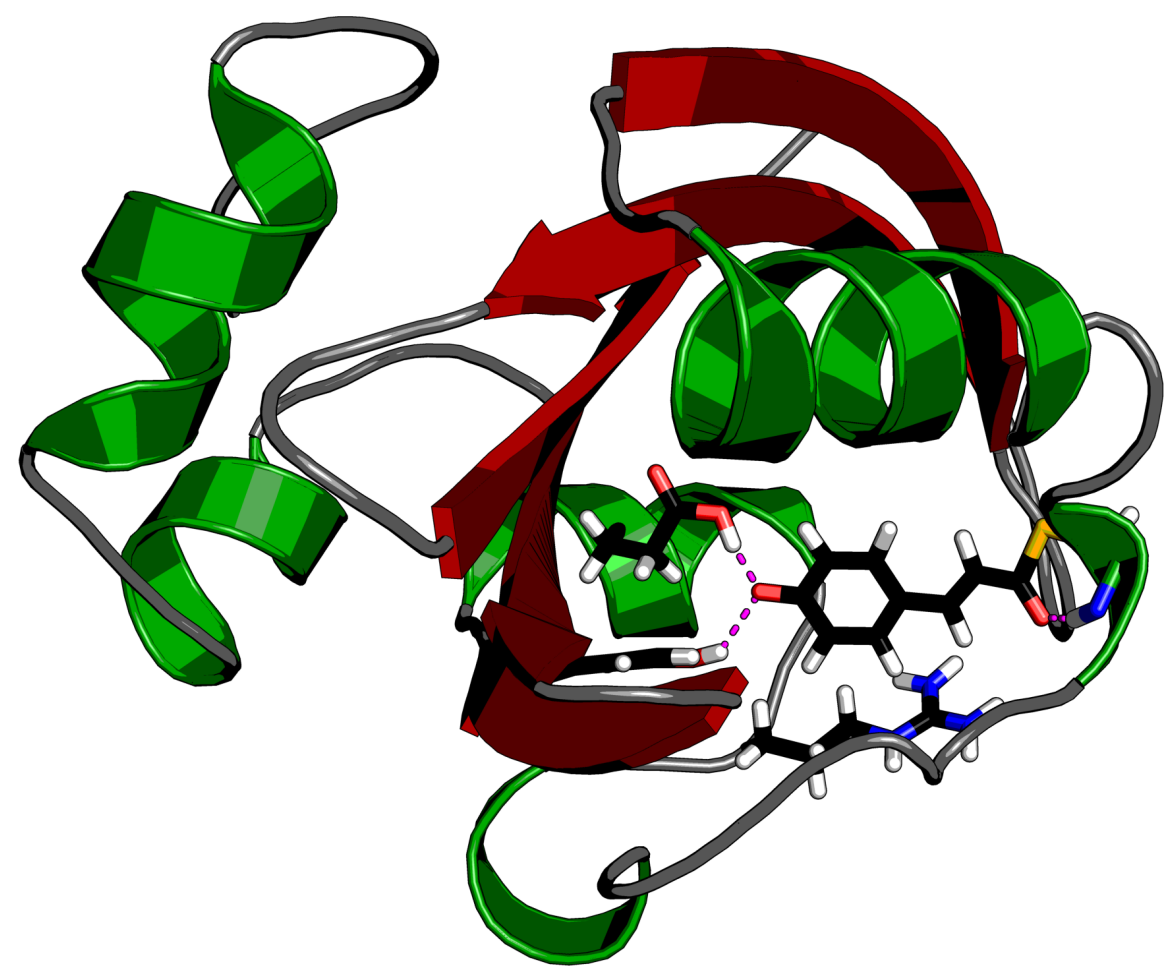

Figure 9.5: The photoactive yellow protein. The chromophore and the active site are shown in the "sticks" representation.

in Figure 9.6. In the resting state of the chromophore, the $\mathrm{C}_{2}-\mathrm{C}_{3}$ double bond (red) adopts the trans-conformation (for the atom names and the definition of the bond color coding consider Figure 9.7). After absorption of a blue light photon, in the resting state $(\mathrm{pG})$ a pre-rotation of the double bond to $90^{\circ}$ takes place. The molecule then encounters a conical intersection and a radiationless transition from the excited $S_{1}$ to the ground state $S_{0}$ occurs. After the $S_{1} / S_{0}$ transition, the double bond either rotates back to its initial trans-conformation or the double bond rotates further to adopt the cis-conformation $(\mathrm{pR})$. These isomerization processes

\footnotetext{
${ }^{1}$ Protein data base (PDB) code $2 \mathrm{ZOH}$
} 


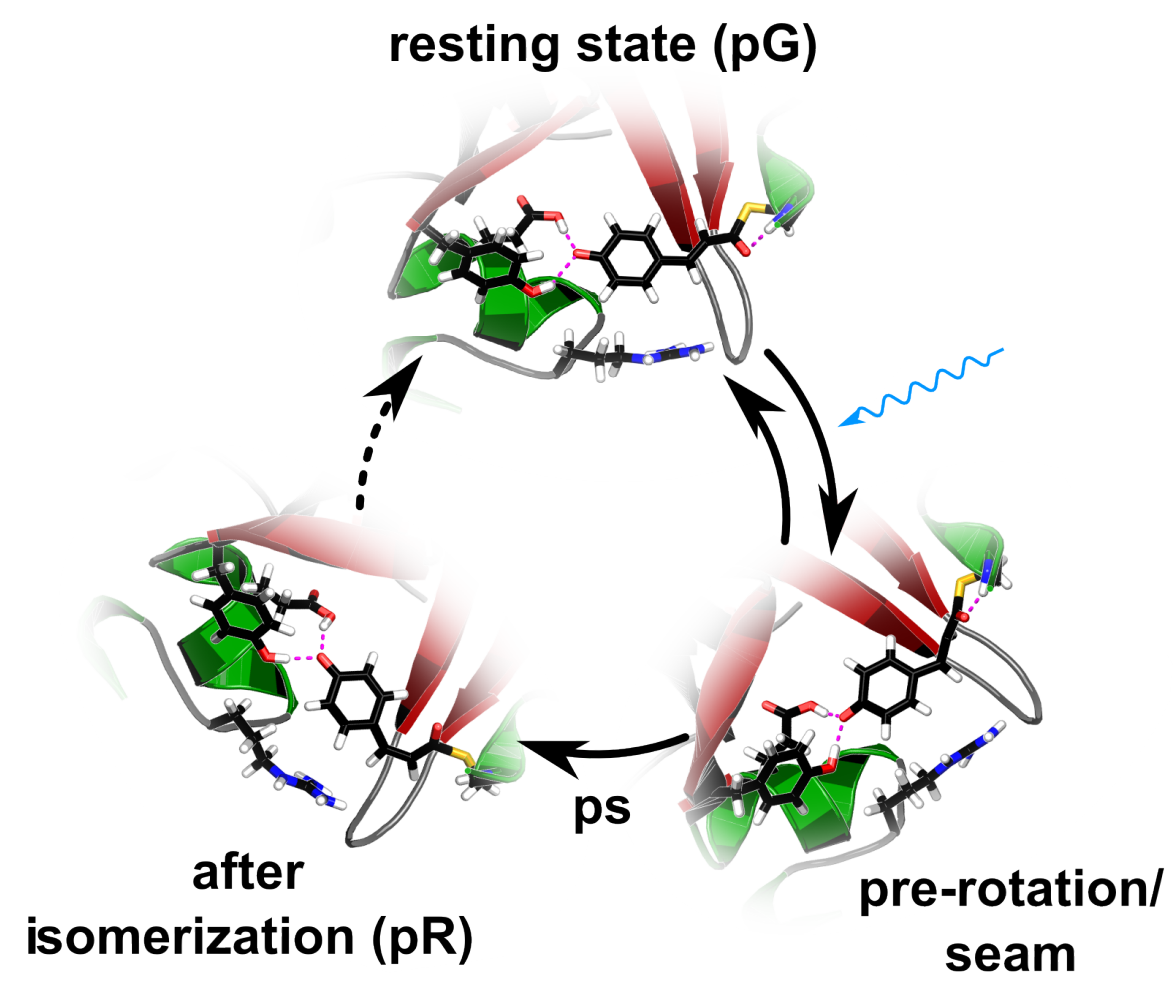

Figure 9.6: Schematic view of the early phase of the photoactive yellow protein photocycle[31, 36].

take place on a picosecond timescale[35]. After the isomerization the protonation state of the chromophore changes triggering structural change. The change in structure is believed to induce the signal transduction[32, 35].

The full photocycle is beyond the reach of excited state MD simulations due to its long timescale. Previous computational studies[36] and also the current work focus on the first part of the photocycle namely the $S_{1} / S_{0}$ transition and the following isomerization.



Figure 9.7: Definition of the atom names. The colors of the dihedral angles will be used in the following. 
First the setup used for the excited state simulations is discussed (cf. Section 9.2.1). In following section the isomerization pathways are discussed on the basis of three specific trajectories. In Section 9.2.3 the results of all simulations are summarized and discussed.

\subsubsection{Setup}

The excited state simulations were started from the same initial configurations as in Reference[36] which were kindly provided by Dr. Gerrit Groenhof ${ }^{1}$ In contrast to Reference[36] the Ambero3[142] force field (FF) with the TIP 3 P water model[154] and the larger $6-31 G^{*}$ basis $^{2}[85,86]$ was used. Furthermore, two different active spaces CAS $(10,9)$ and $C A S(8,7)$ were employed in the quantum mechanics $(Q M)$ part of the simulations. The MOs composing the active spaces were derived by starting from the CAS(12,11) including all $\pi$ MOs except for the $3 \pi \mathrm{MO}$ of the sulfur. Then the $\pi \mathrm{MO}$ with the most or the least nodes was removed from CAS and the wavefunction converged. This was repeated until converged, state averaging (SA)2-CASSCF $(10,9)$ and SA2-CASSCF $(8,7)$ wavefunctions were obtained.

For each initial configuration, six trajectories were started. One for each of the hopping algorithms discussed in Sections 8.3 and 8.4, using both active spaces discussed above. For all trajectories run with the FSSH algorithms starting from the same initial configuration the random number generation was seeded the same. This allows a comparison of the influence of the two active spaces on the excited state dynamics.

\subsubsection{Excited State Dynamics}

Figure 9.8 shows a trajectory run with the DSH algorithm with the CAS $(10,9)$ that undergoes a trans-to-cis isomerization of the $\mathrm{C}_{2}-\mathrm{C}_{3}$ double bond. The trajectory follows the pathway sketched in Figure 9.6. The chromophore is excited to its excited state at $t=0 \mathrm{ps}$. Right after the excitation the $\mathrm{C}_{2}-\mathrm{C}_{3}$ double bond dihedral (green line) starts to pre-twist to about $100^{\circ}$. The hop to the ground state takes place at around $t=0.2 \mathrm{ps}$ (vertical black line). After the hop at about $t=0.3 \mathrm{ps}$, the double bond twists further reaching its cis-conformation at around $t=0.5 \mathrm{ps}$. At the same time the $\mathrm{S} \gamma-\mathrm{C}_{1}$ dihedral starts rotating. The oxygen $\mathrm{O}_{1}$ moves away from the Cys amine group breaking the hydrogen bond. These dynamics resemble

${ }^{1}$ Nanoscience Center, University of Jyäskylä

${ }^{2}$ The cartesian variant of the basis was used as available in the Molpro 2009.1 program. 


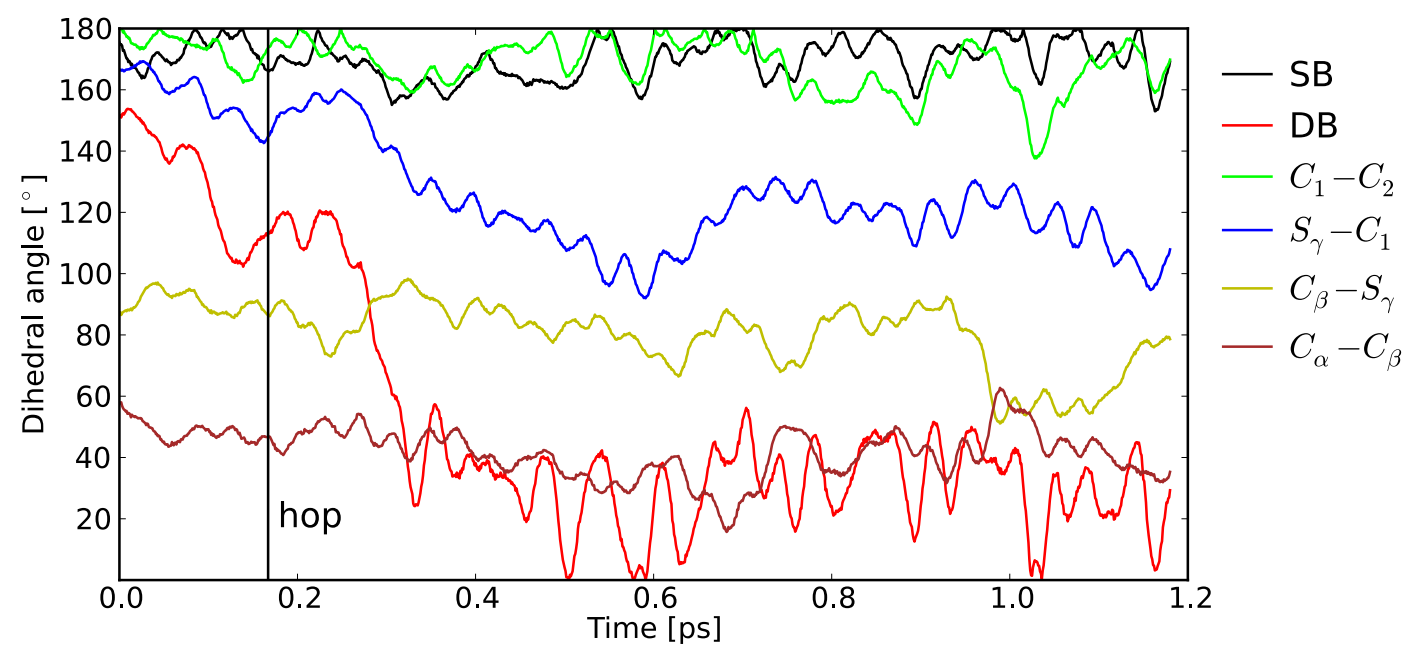

Figure 9.8: The DSH CAS $(10,9)$ trajectory 1 showing a trans-to-cis isomerization of the $\mathrm{C}_{2}-\mathrm{C}_{3}$ double bond. The dihedrals along different bonds in the chromophore are shown. The vertical black line indicates the hop from the excited to the ground state.

the isomerization dynamics described in Reference[36]. However, no flip of the thioester linkage $\left(\mathrm{C} \beta-\mathrm{S} \gamma-\mathrm{C}_{1}\right)$ was observed here as there is no rotation around $\mathrm{C} \alpha-\mathrm{C} \beta$ bond.

In addition to the isomerization of the double bond, also single bond $\left(\mathrm{C}_{1},-\mathrm{C}_{3}\right)$ isomerization was observed. Figure 9.9 shows the $\operatorname{CAS}(8,7)$ trajectory 6 which exhibits a trans-to-trans single bond isomerization. Again, a pre-twisting takes place prior to the hop to the ground state. In this case, however, the single bond (black line) rotates to $90^{\circ}$ prior to the surface hop. The rotation of the single bond corresponds to a rotation of the phenyl ring with respect to the rest of the chromophore. This time, it takes the trajectory about $1.2 \mathrm{ps}$ to reach the pre-twisting region. The trajectory hits the seam at around $1.5 \mathrm{ps}$ and hops to the ground state. Right after the hop the single bond rotates back to its trans-conformation reassuming its initial structure.

The FSSH algorithms use a stochastic criterion to determine the hop. Thus the hop can happen essentially anywhere along the trajectory. Figure 9.10 shows the FSSH CAS $(8,7)$ trajectory 6 . The trajectory hops (black vertical line) at around $t=0.2 \mathrm{ps}$ before any pre-twisting of the single bond (black line) or the double bond (red line) occurs. The instantaneous hopping probability $p_{n}$ (see Figure 9.10 (bottom)) is below 0.1 at the hop. Right after the hop, the instantaneous hopping 


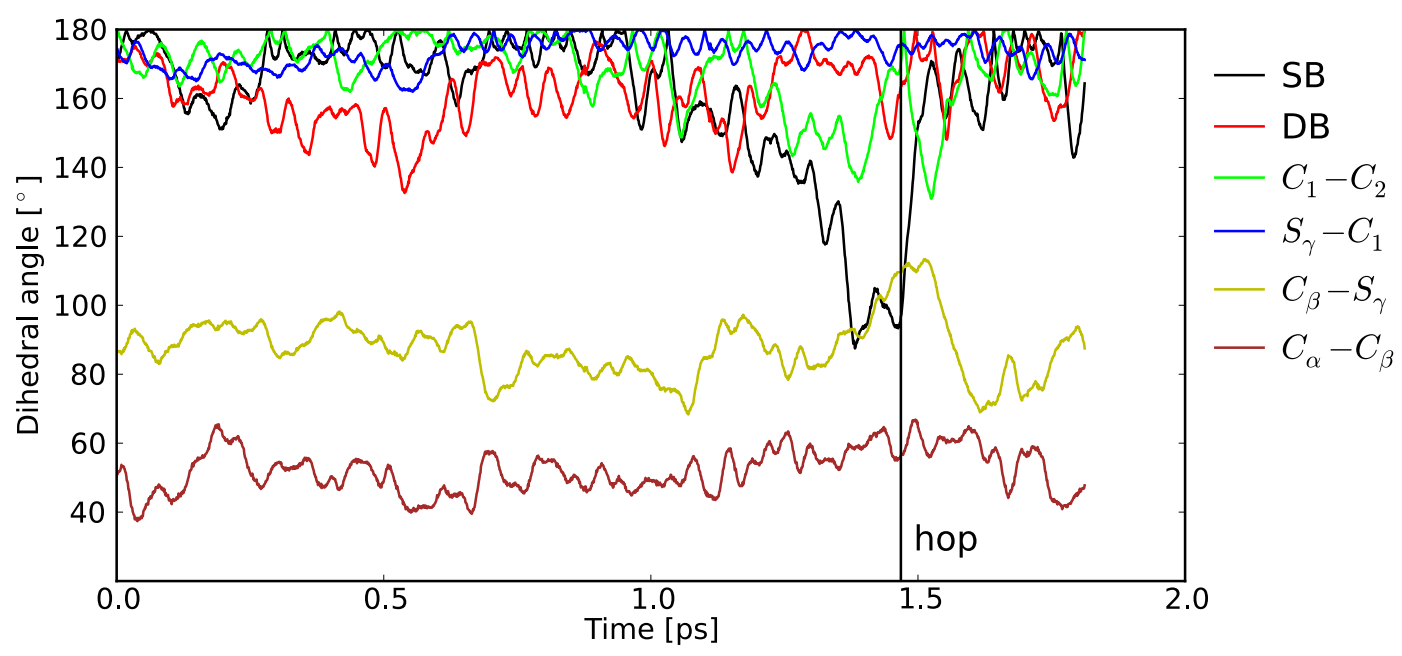

Figure 9.9: The DSH CAS $(8,7)$ trajectory 6 showing a trans-to-trans isomerization of the $\mathrm{C}_{1},-\mathrm{C}_{3}$ single bond. The dihedrals along different bonds in the chromophore are shown. The vertical black line indicates the hop from the excited to the ground state.

probability back to the excited state is large until the end of the simulation. The hop back to the excited state does not occur, though, because it is energetically forbidden. The trajectory is essentially trapped in the electronic ground state by the enforced energy conservation. As the trajectory cannot hop back to the excited state, it does not properly represent the wavefunction used in the FSSH algorithm anymore.

\subsubsection{Summarized Results}

To access whether a FSSH trajectory hops before reaching a region of strong nonadiabatic coupling and potentially suffers from the trapping problem described above, the accumulated hopping probability

$$
P_{\text {hop }}=1-\prod_{n=0}^{N_{\text {hop }}}\left(1-p_{n}\right)
$$

is used. The accumulated hopping probability describes the probability of a trajectory having hopped to the ground state up to the the timestep the hop actually takes place. 




Figure 9.10: The FSSH CAS $(8,7)$ trajectory 6. The dihedrals along different bonds are shown top. In the bottom part the instantaneous hopping probability is plotted.

Table 9.5 summarizes the results of the trajectories run with the CAS $(8,7)$. Only two out of the nine FSSH trajectories reach the pre-twisting of either the single or the double bond and exhibit an isomerization. The rest of the FSSH trajectories hop before reaching the seam and no isomerization is observed. For most of these trajectories the accumulated hopping probability is fairly low, meaning that they all suffer from the trapping problem discussed before. Notably, there are three unproductive FSSH trajectories (FSSH run 6 and FSSH DC runs 6 and 7 ) showing a high accumulated hopping probability without reaching the pre-twisting of either the single or the double bond. This indicates that there is significant nonadiabatic coupling beyond the seam of the conical intersection.

The DC to the FSSH remedies this situation to a certain extend as five out of nine trajectories reach the pre-twisting region.

Both the FSSH and the DSH trajectories show single as well as double bond transto-trans isomerization. Double bond trans-to-cis isomerizations are, however, not observed in either case.

For the FSSH trajectories, the exited state lifetime of the "productive" trajectories is shorter than that of the DSH trajectories as all FSSH trajectories hop back to the 


\begin{tabular}{ccccccccc}
\hline & \multicolumn{3}{c}{ DSH } & \multicolumn{3}{c}{ FSSH } & \multicolumn{3}{c}{ FSSH DC } \\
\hline run & $t_{\text {hop }}$ ps & result & $t_{\text {hop }}$ ps & $P_{\text {hop }}$ & result & $t_{\text {hop }}$ ps & $P_{\text {hop }}$ & result \\
1 & 1.184 & DB trans & 0.434 & 0.98 & DB trans & 0.235 & 0.79 & DB trans \\
2 & 1.303 & DB trans & 0.056 & 0.15 & -2 & 0.590 & 0.62 & SB trans \\
3 & 1.213 & DB trans & 0.027 & 0.22 & $-^{2}$ & 0.027 & 0.18 & $-^{2}$ \\
4 & 1.532 & DB trans & 0.152 & 0.26 & $-^{2}$ & 0.769 & 0.88 & DB trans \\
5 & 3.008 & -1 & 0.230 & 0.92 & DB trans & 0.657 & 1.00 & DB trans \\
6 & 1.468 & SB trans & 0.214 & 0.72 & -2 & 0.214 & 0.80 & $-^{2}$ \\
7 & 0.947 & SB trans & 0.011 & 0.06 & $-^{2}$ & 0.761 & 1.00 & $-^{2}$ \\
8 & 2.649 & -1 & 0.003 & $0.00^{3}$ & $-^{2}$ & 0.003 & $0.00^{3}$ & $-^{2}$ \\
9 & 0.811 & SB trans & 0.046 & 0.08 & DB trans & 0.339 & 1.00 & SB trans \\
\hline
\end{tabular}

Table 9.5: Summary of the results obtained with the CAS $(8,7)$.

${ }^{1}$ The trajectory has not hopped yet. The time stated is the current simulation time as of the 07.02.2013. This time is a lower bound for the hopping time.

2 The hop occurred before the pre-twisting of either the single or the double bond. Hence, no isomerization was observed.

3 The hopping probability is less then 0.005 .

ground state in less than 0.8 ps while the DSH trajectories stay in the excited state for at least 0.9 ps.

Table 9.6 summarizes the results of the trajectories run with the larger CAS $(10,9)$. Unfortunately, most of the DSH trajectories have not reached the seam region yet. Thus, these trajectories have not hopped to the ground state and the only information drawn from these trajectories is a lower bound of their excited state lifetime.

Again, only two of the FSSH trajectories show an isomerization. Here, the DC scheme cannot really remedy the trapping problem as only three of the nine FSSH DC trajectories undergo an isomerization. Similar to the CAS $(8,7)$ simulations the trajectories run with either FSSH algorithm suffer from the trapping problem.

Like in the CAS $(8,7)$ there are some FSSH trajectories (FSSH runs 5 and 6 and FSSH DC run 7) that have a large accumulated hopping probability at the hop without actually reaching the seam region. Hence, also the CAS(10,9) PES seems to have regions of nonadiabatic coupling except for the seam itself.

A thorough comparison of FSSH to the DSH trajectories for the CAS(10,9) is not possible because most of the DSH have not reached the seam of the conical intersection yet. The excited state lifetimes of the DSH trajectories are going to be 


\begin{tabular}{ccccccccc}
\hline & \multicolumn{3}{c}{ DSH } & \multicolumn{3}{c}{ FSSH } & \multicolumn{3}{c}{ FSSH DC } \\
\hline run & $t_{\text {hop }}$ & result & $t_{\text {hop }}$ & $P_{\text {hop }}$ & result & $t_{\text {hop }}$ & $P_{\text {hop }}$ & result \\
1 & 0.235 & DB cis & 0.154 & 0.82 & DB trans & 0.226 & 0.81 & DB trans \\
2 & 1.947 & $-^{1}$ & 0.056 & 0.17 & $-^{2}$ & 0.165 & 0.048 & $-^{2}$ \\
3 & 1.886 & $-^{1}$ & 0.027 & 0.21 & $-^{2}$ & 0.027 & 0.20 & $-^{2}$ \\
4 & 1.899 & -1 & 0.179 & 0.46 & SB trans & 0.569 & 0.32 & SB trans \\
5 & 1.488 & -1 & 0.923 & 0.99 & $-^{2}$ & 1.626 & 0.99 & DB trans \\
6 & 1.097 & SB trans & 0.385 & 0.89 & $-^{2}$ & 0.214 & 0.75 & $-^{2}$ \\
7 & 1.445 & -1 & 0.404 & 0.94 & $-^{2}$ & 1.859 & - & $-^{1}$ \\
8 & 1.649 & -1 & 0.003 & $0.00^{-1}$ & $-^{2}$ & $0.00^{4}$ & 0.002766 & $-^{2}$ \\
9 & -3 & - & 0.046 & 0.095642 & $-^{2}$ & 0.164 & 0.17 & $-^{2}$ \\
\hline
\end{tabular}

Table 9.6: Summary of the results obtained with the CAS(10,9).

1 The trajectory has not hopped yet. The time stated is the current simulation time as of the 07.02.2013. This time is a lower bound for the hopping time.

2 The hop occurred before the pre-twisting of either the single or the double bond. Hence, no isomerization was observed.

3 Trajectory is broken.

4 The hopping probability is less then 0.005 .

larger than the FSSH excited lifetimes, because the current DSH simulation times are already larger than FSSH lifetimes.

Despite the unproductive trajectories encountered with the FSSH algorithms the isomerization mechanism is the same for all hopping algorithms and CASs. Isomerization can only occur if the trajectory reaches the region of pre-twisted single or double bond dihedral.

Unfortunately, there is only one trajectory showing the trans-to-cis isomerization. This trajectory, indeed, shows that the isomerization pathway found in Reference [36] with a smaller active space and a smaller basis set $(3-21 \mathrm{G})$ also occurs with the large CAS $(10,9)$ with the $6-31 G^{*}$ basis set.

One should note, however, that the statistics are rather limited. Especially no trans-to-cis isomerization were observed in the $\operatorname{CAS}(8,7)$ simulations. Hence, we do not make any quantitative predictions of the excited state lifetimes or quantum yields. 


\subsection{Conclusion}

In the PSB2 simulations, there is only a small difference between the results obtained with the DSH and the FSSH algorithms. The model free estimation of the excited state lifetimes does not yield a significant difference among the different hopping algorithms.

That all three hopping algorithms predict the same reaction mechanism and products is probably caused by the excited state dynamics of the PSB2. The trajectories follow a "down hill" pathway without any barriers in the $S_{1}$ PES or equilibration in a $S_{1}$ minimum. Hence, there does not seem to be a region of strong nonadiabatic coupling except for the conical intersection itself. Furthermore, the seam is usually reached in less than $200 \mathrm{fs}$. There is simply little time for any deviations due to the wavefunctions propagation used in the FSSH algorithms.

Due to the specific excited state dynamics of the PSB2, the small differences found among the hopping algorithms cannot be generalized to different systems which do not follow a similar "down hill" process, e.g. the PYP. Thus, the PSB2 is probably not a good test system to judge the applicability of the DSH and FSSH algorithms to molecules which exhibit a more complex excited state MD.

For the PYP simulations the situation is different. The current implementation of the FSSH algorithms used in this work enforces energy conservation during the hops by adjusting the kinetic energy accordingly. This implementation cause the trapping of several FSSH trajectories in ground state because the hop back to the excited state is energetically forbidden despite a large hopping probability. In such cases, the time evolution of the trajectory does not describe the system properly anymore as the FSSH algorithms predicts a time-evolution according to the excited state PES.

The DC scheme does not entirely solve this problem because in the CAS(10,9) simulations most of the trajectories hop prior to the pre-twisting and at large $S_{1}-S_{0}$ energy gaps still. An obvious solution to the frustrated hop problem is enhanced sampling, i.e. starting with multiple trajectories with different seeds for the random number generator for each initial condition. Especially in the case of the CAS(10,9), the quantum mechanics/molecular mechanics (QM/MM) calculations are expensive. So, just increasing the number of trajectories is not practical.

For the two systems studied in this work, there is little incentive of choosing either of the FSSH algorithms in the specific implementation used here over the DSH algorithm. In the PSB2 simulations, there is only a small difference among the different hopping algorithms. For the PYP, the trapping problem diminishes 
the statistics achieved with the FSSH algorithms compared to the DSH algorithm. Hence, the use of the DSH seems preferable in this case as well.

For systems where the nonadiabatic coupling is essentially a located conical intersection, the DSH algorithm seems to be preferable. However, there might be situations where the excited state dynamics are dominated by extended regions of nonadiabatic coupling without an actual conical intersection. For such a system the DSH does not describe the excited state MD properly because the DSH only describes hops at conical intersections.

Due to the trapping problem found for the implementation of the FSSH algorithms used in this work, a different scheme to cope with the energy conservation in the FSSH algorithms[155] or a different simulation protocol should be used[156]. 



\section{0 \\ Excitation Energy Transfer}

Excitation energy transfer (EET) processes take place in systems containing two or more chromophores. The excitation is transferred from the excited state donor to the ground state acceptor by coupling between the two chromophores. Foerster resonance energy transfer (FRET)[40] is an example of an EET process, which forms the basis for the experimental technique with the same name[42, 43]. In FRET the donor absorbs a photon but the excitation energy is released by photo-emission from the acceptor. To facilitate FRET, the excitation has to be transferred from one part of the system to another. In FRET theory[40], as well as for instance in Dexter's theory[41], the excitation transfer is attributed to weak interaction between the (distinct) wavefunctions of the two chromophores. The interaction between the donor and the acceptor is, hence, treated in terms of perturbation theory. In FRET theory, the coupling derived by Fermi's golden rule is further approximated to the dipole-dipole coupling between the transition dipole moments of the wavefunctions of the two chromophores.

In contrast to the conventional interpretation of EET in terms of perturbation theory, it has been proposed [44-47] that in small molecules nonadiabatic coupling between different excited state potential energy surface (PES) (cf. Chapter 7) can induce the intra-molecular EET. There are two plausible pathways by which molecular dynamics (MD) through regions of nonadiabatic could lead to EET.

The first pathway is a "down hill" process, in which the nuclear wavepacket passes from the $S_{2}$ through a conical intersection to the $S_{1}$. Figure 10.1 sketches a PESs which can induce an EET process. The nuclear wavepacket is excited from the ground state (black) to the second excited state (red), for which the excitation is located on the left part of the molecule. The wavepacket follows the red surface until it reaches the conical intersection. Depending on the detailed shape of the conical intersection, a part of the wavepacket will follow the red surface diabatically reach the minimum of the red surface. The other part of the wavepacket, however, will 




Figure 10.1: Sketch of two excited potential energy surfaces leading to a "down hill" excitation energy transfer. The potential energy surfaces are colored according to the diabatic states.

be transferred to the other (blue) surface. For the blue surface, the excitation is located on the right side of the molecule. Thus, the nonadiabatic coupling between different PESs induces MD that leads to an EET.

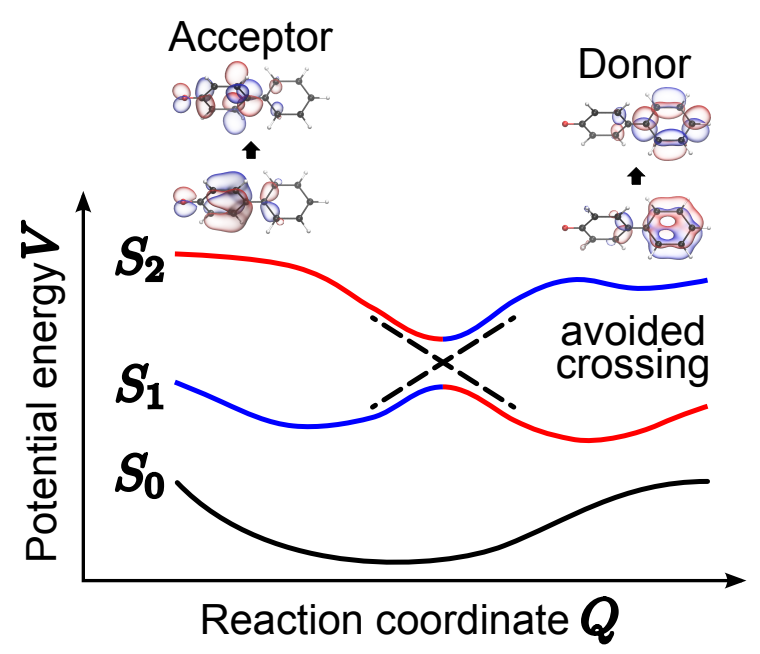

Figure 10.2: Sketch of two excited potential energy surfaces leading to a excitation energy transfer by excited state barrier crossing. The potential energy surfaces are colored according to the diabatic states. 
The second possibility is sketched in Figure 10.2. Here, the $S_{1}$ surface exhibits a barrier, which is caused either by an avoided crossing (shown) with the $S_{2}$ or a conical intersection between the $S_{1}$ and the $S_{2}$. Due to the nonadiabatic coupling, the excited states exchange their diabatic character. The wavepacket is now excited from the ground state PES to the $S_{1}$ blue surface for which the excitation is located on the right end of the molecule. Part of the wavepacket can now cross the barrier reaching the red surface by thermal activation which transfers the excitation to the left side of the molecule. Again, the nonadiabatic coupling shapes the PESs so that the MD can induce EET from one part of the molecule to the other.

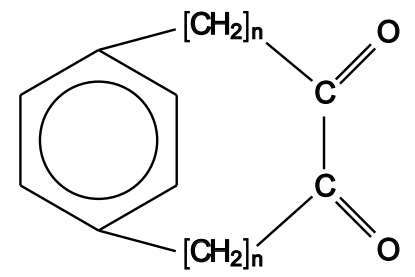

Figure 10.3: Pnn structure.

Speiser and co-workers[48] used spectroscopy to study the intra-molecular EET for a set of bi-chromophoric p-cyclophanes (Pnn, see Figure 10.3), m-cyclophanes $(\mathrm{M} n n)$, and benzocycloalkenediones $(\mathrm{O} n n)$. The absorption spectra of these compounds are dominated by the absorption spectrum of the xylene, as the $\left(n, \pi^{*}\right)$ excitation of the bi-acetyl is essentially optically dark. The emission spectrum of e.g. the $\mathrm{P}_{44}$ is, however, essentially the emission spectrum of the bi-acetyl. Thus, the excitation energy has to be transferred from the xylene to the bi-acetyl. The authors attribute the EET to a process driven by short range exchange interaction between the chromophores and interpret their results in terms of Dexter's theory accordingly.

The distance between the xylene and the bi-acetyl is about $0.5 \mathrm{~nm}$. The perturbative treatment of the interaction between the chromophores is somewhat artificial as it assumes two distinct wavefunctions. Instead, we here propose to to study the excited state MD of P44 using one wavefunction for the whole molecule to reveal possible MD induced EET mechanism.

Accordingly, we have performed both ab initio calculations as well as excited state MD simulations to investigate a possible MD induced EET process in $\mathrm{P}_{44}$. The ab initio calculations (especially the optimization of the conical intersections) were performed by Dr. Martial Boggio-Pasqua ${ }^{1}$ who kindly provided his data.

\footnotetext{
${ }^{1}$ Laboratoire de Chimie et Physique Quantiques, Université de Toulouse
} 
First, the setup used for our calculations is discussed in the following section. Then the minimal energy pathway (MEP) is presented on the basis of the ab initio results (cf. Section 10.2.1). In the Sections 10.2.2 and 10.2.3 the results of the excited state MD are considered and compared to the MEP.

\subsection{Setup}

The initial conditions for the quantum mechanics/molecular mechanics (QM/MM) dynamic simulations are determined from a B3LYP $[157-160] / 6-31 G^{*}[85,86]$ trajectory run on the ground state. From this trajectory snapshots were taken every $1 \mathrm{~ns}$. At these snapshots the electronic wavefunction is vertically excited to the $\pi-\pi^{*}$ state. The $\pi-\pi^{*}$ is either of the $S_{2}$ or $S_{3}$ state, depending on the molecular geometry.

The dynamics were run using state averaging (SA)4-complete active space selfconsistent field (CASSCF) $(14,12) / 6-31 G^{* 1}$ for the dryad and the Gromos43a2[140, 141] force field (FF) for the hexane solvent. As hexane is apolar, there are no charges from the MM region polarizing the wavefunction. A timestep of $1 \mathrm{fs}$ was used for the MD. The setup was done mostly by Dr. Gerrit Groenhof ${ }^{2}$ who kindly provided me his data.

One successful trajectory was run with the diabatic surface hopping algorithm which happens to follow the MEP suggested by the ab initio calculations.

The fewest switches surface hopping (FSSH) was used despite the poor performance seen in the photoactive yellow protein (PYP) simulations (cf. Section 9.2) because the choice of the hopping algorithm might influence the observed pathway (cf. the discussion in Section 10.2.1).

Only 3 out of 10 trajectories started with the fewest switching surface hopping algorithm were successful. For the other starting geometries it was not possible to derive the state character from the transition density matrices due to "broken" orbital subspace. Hence, it was impossible to start the trajectories on the correct $\left(\pi, \pi^{*}\right)$ state.

The successful trajectories were stopped shortly after reaching the fluorescent $S_{1}\left(n, \pi^{*}\right)$ surface.

${ }^{1}$ The cartesian variant of the basis was used as available in the Molpro 2009.1 program.

${ }^{2}$ Nanoscience Center, University of Jyväskylä 


\subsection{Results}

\subsubsection{Ab Initio Results}

\begin{tabular}{ccc}
\hline Intersecting surfaces & Relative energy [ev] & Dimensionality of the seam \\
\hline$S_{1}\left(n, \pi^{*}\right) / S_{2}\left(n, \pi^{*}\right)$ & $5.972 / 5.986$ & $n-2$ \\
$S_{2}\left(n, \pi^{*}\right) / S_{3}\left(\pi, \pi^{*}\right)$ & $4.750 / 4.784$ & $n-1$ \\
$S_{1}\left(n, \pi^{*}\right) / S_{2}\left(\pi, \pi^{*}\right)$ & $4.829 / 4.899$ & $n-2$ \\
\hline
\end{tabular}

Table 10.1: Minimal energy conical intersections between the electronic states for the $C_{2}$ geometries. The energies are given with respect to the ground state energy minimum. The two energies are the energies of the two states involved in the conical intersection a its location. The seam of the $S_{2}\left(n, \pi^{*}\right) / S_{3}\left(\pi, \pi^{*}\right)$ conical intersection is $n-1$ dimensional because the gradient difference and derivative coupling vectors are parallel and, thus, the branching space is one dimensional.

We located conical intersections between all three excited states (see Table 10.1). The vertical excitation energy from the ground to the $S_{3}\left(\pi, \pi^{*}\right)$ state is $4.966 \mathrm{eV}$. So, at least the $S_{2}\left(n, \pi^{*}\right) / S_{3}\left(\pi, \pi^{*}\right)$ and the $S_{1}\left(n, \pi^{*}\right) / S_{3}\left(\pi, \pi^{*}\right)$ are in principle energetically accessible. The conical intersection between the $S_{2}\left(n, \pi^{*}\right)$ and the $S_{3}\left(\pi, \pi^{*}\right)$ state is $n-1$ dimensional because the gradient difference and the derivative coupling vectors are parallel and, thus, the branching space is one dimensional.

The ab initio data suggests an MEP sketched in figure 10.4. Starting from the $S_{0}$ (black curve) minimum the system is excited to the $S_{3}\left(\pi, \pi^{*}\right)$ state (green curve). The trajectory then travels towards the minimum of the potential energy surface of the $\left(\pi, \pi^{*}\right)$ state. The trajectory crosses the $S_{2}\left(n, \pi^{*}\right) / S_{3}\left(\pi, \pi^{*}\right)$ conical intersection without hopping to the second $\left(n, \pi^{*}\right)$ state (blue curve), because the seam of this conical intersection is $n-1$ dimensional. When the system hits the $S_{1}\left(n, \pi^{*}\right) / S_{2}\left(\pi, \pi^{*}\right)$ conical intersection, it can hop to the $S_{1}\left(n, \pi^{*}\right)$ (red curve) as the seam is $n-2$ dimensional. It then relaxes on the $S_{1}\left(n, \pi^{*}\right)$ potential energy surface until the fluorescent decay to the ground state takes place.

Note, however, that the pathway sketched above assumes that the hopping algorithm sticks to the instantaneous diabatic surface while passing through the conical intersection. This is certainly true for the diabatic surface hopping (DSH) algorithm which can only pass from one diabatic state to another in the vicinity of a two-dimensional conical intersection (see Figure 10.5). 


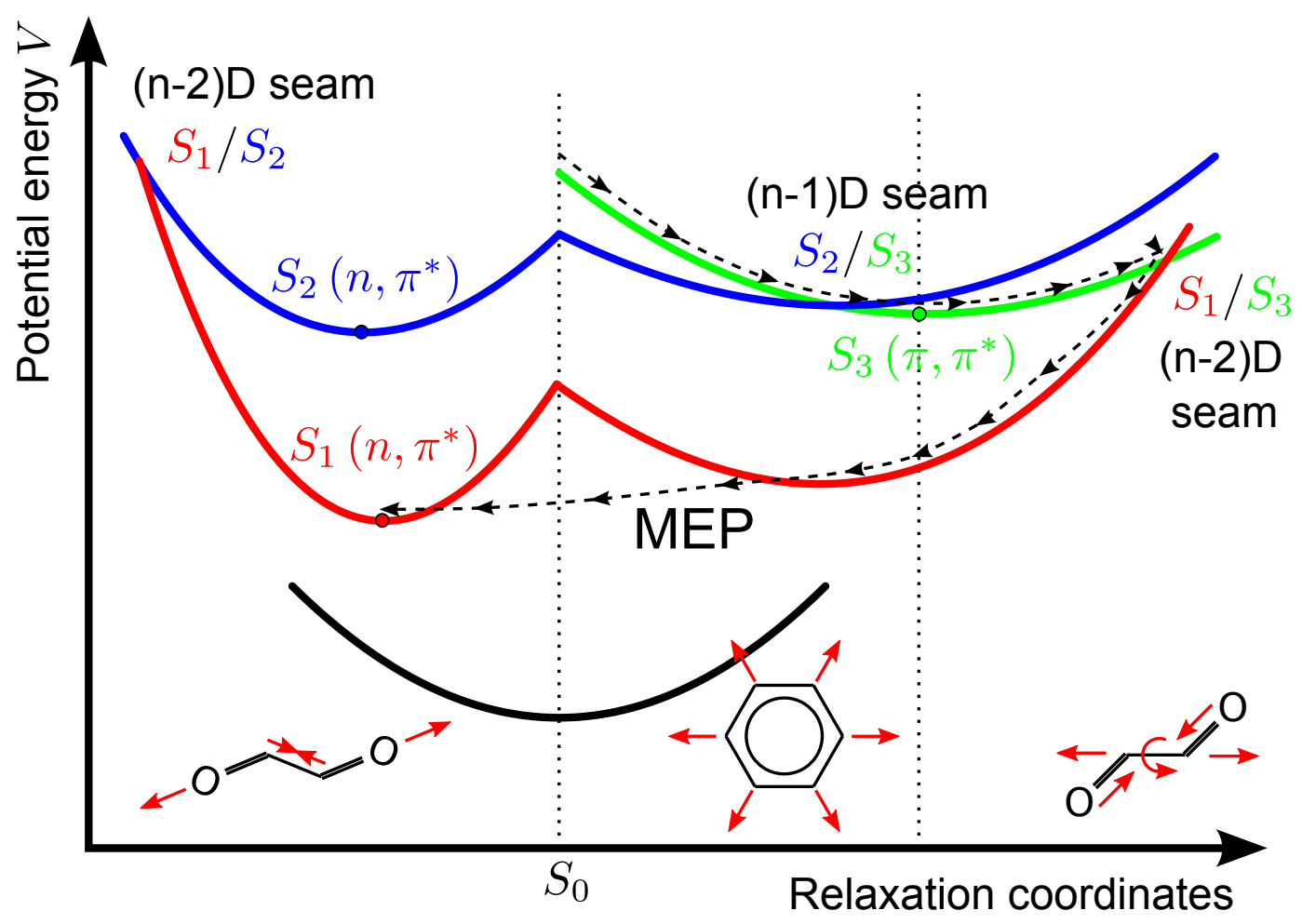

Figure 10.4: Sketch of the minimal energy pathway of the excitation energy transfer reaction derived from the $\operatorname{CASSCF}(14,12) / 6-31 G^{*}$ ab initio calculation. Note that only single point calculations were performed. Thus, only the minimal energy points of the respective states and the conical intersection are known. No information about the curvature or shape of the potential energy surfaces were obtained and the curves are merely to guide the eye. In the sketch not a single reaction coordinate is used, but there are three different motions connecting the different energy minima. The different states are labeled according to their ordering at the ground state energy minimum geometry.

The hops in the FSSH algorithm, however, are determined from the strength of the nonadiabatic coupling and the hops can essentially occur anywhere along the trajectory. The seam of the $S_{2}\left(n, \pi^{*}\right) / S_{3}\left(\pi, \pi^{*}\right)$ conical intersection is $n-1$ dimensional because the derivative coupling and the gradient difference vectors are parallel. Hence, there still is nonadiabatic coupling between the $S_{2}$ and $S_{3}$ surfaces. 


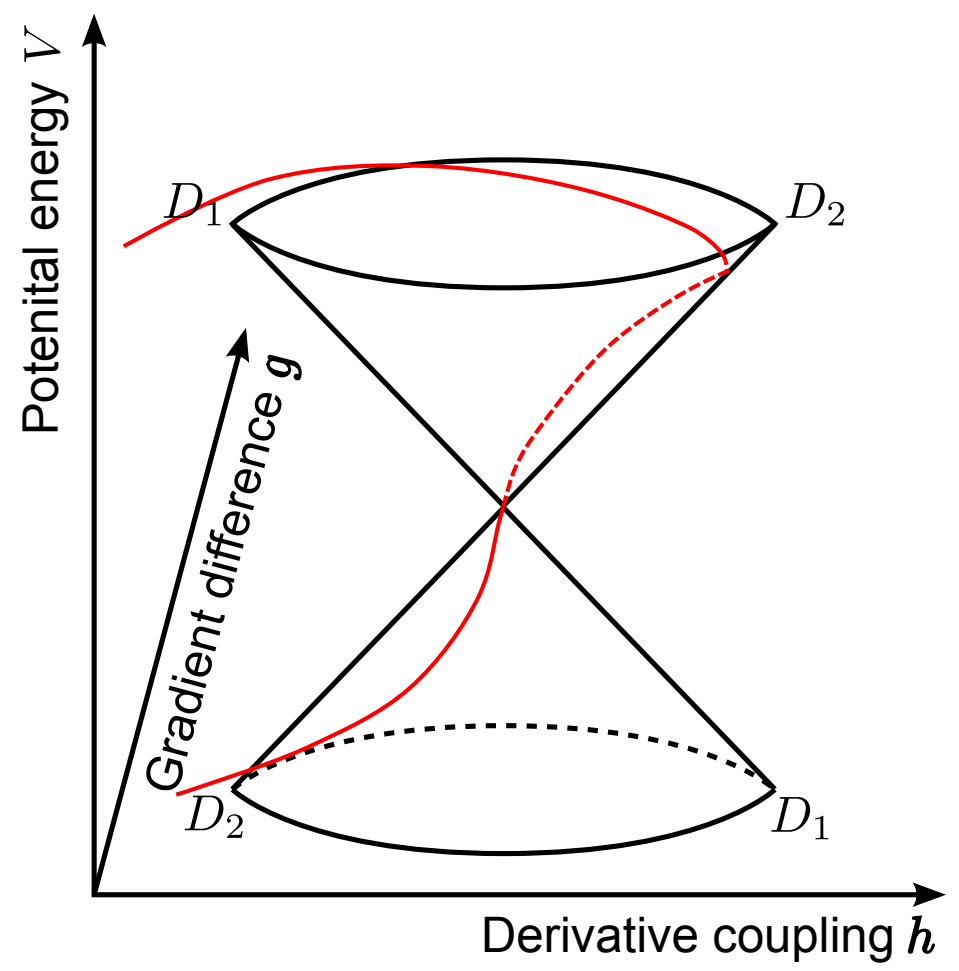

Figure 10.5: DSH trajectory switching diabatic state while evolving around a conical intersection. Before the trajectory reaches the conical intersection itself, it switches from one diabatic state $D_{1}$ to the other diabatic state $D_{2}$ by moving around the upper cone. Then, it switches to the lower surface by evolving through the conical intersection.

So, the FSSH trajectories will not necessarily follow the pathway described above but rather hop to the $S_{2}\left(n, \pi^{*}\right)$ rather than following the diabatic $\left(\pi, \pi^{*}\right)$ state.

The de-excitation pathway that leads the system from the $S_{3}$ surface to the fluorescent $S_{1}$ surface might, thus, depend on the surface hopping algorithm used in the simulations. Therefore, trajectories with both the DSH as well as the FSSH algorithms were run.

The MEP sketched in Figure 10.4 implements the "down hill" EET mechanism discussed in Figure 10.1 but with three instead of two excited states determining the excited state dynamics.

This MEP explains the experimental findings [exp. paper] that the absorption takes place at a wavelength corresponding to the $\pi-\pi^{*}$ transition on the xylene motif whereas the emission wavelength corresponds to the $n-\pi^{*}$ transition on the 
di-acetyl motif. The excitation energy transfer from the xylene to the di-acetyl is, thus, explained by the molecular dynamics on the excited state potential energy surfaces and the nonadiabatic coupling among them.

\subsubsection{DSH Trajectory}

\begin{tabular}{cll}
\hline Time [fs] & Transition & $\begin{array}{l}\text { Angle between derivative } \\
\text { coupling and gradient dif- } \\
\text { ference vector }\end{array}$ \\
\hline 5 & $S_{2}\left(\pi, \pi^{*}\right) \rightarrow S_{3}\left(\pi, \pi^{*}\right)$ & $30.9^{\circ}$ \\
10 & $S_{3}\left(\pi, \pi^{*}\right) \rightarrow S_{2}\left(\pi, \pi^{*}\right)$ & $78.6^{\circ}$ \\
18 & $S_{2}\left(\pi, \pi^{*}\right) \rightarrow S_{1}\left(n, \pi^{*}\right)$ & $172.4^{\circ}$ \\
\hline
\end{tabular}

Table 10.2: Conical intersections encountered in the diabatic surface hopping trajectory.

The trajectory was started on the $S_{2}\left(\pi, \pi^{*}\right)$ state. After $5 \mathrm{fs}$ the trajectory reaches the $S_{2}\left(\pi, \pi^{*}\right) / S_{3}\left(n, \pi^{*}\right)$ conical intersection (figure 10.6). It stays on the $\left(\pi, \pi^{*}\right)$ state while hopping to the $S_{3}$ surface. There the trajectory proceeds until it hits the $S_{2}\left(\pi, \pi^{*}\right) / S_{3}\left(n, \pi^{*}\right)$ conical intersection again after $11 \mathrm{fs}$. Again, the trajectory stays in the $\left(\pi, \pi^{*}\right)$ state in hopping back to the $S_{2}$ surface. After $17 \mathrm{fs}$, the trajectory reaches the $S_{1}\left(n, \pi^{*}\right) / S_{2}\left(\pi, \pi^{*}\right)$ conical intersection and hops to the $S_{1}\left(n, \pi^{*}\right)$ surface. After the first hop after $5 \mathrm{fs}$, the trajectory resembles the MEP pictured in figure 10.4. However, the topology of the conical intersections is not the same as in the MEP (see table 10.2). The trajectory hits the $S_{2}\left(n, \pi^{*}\right) / S_{3}\left(\pi, \pi^{*}\right)$ twice (after $5 \mathrm{fs}$ and $10 \mathrm{fs}$ ). In both cases the seam is $n-2$ dimensional. This means that the DSH simulations as well are not bound to stay on the $\left(\pi, \pi^{*}\right)$ state while passing through the $S_{2}\left(\pi, \pi^{*}\right) / S_{3}\left(n, \pi^{*}\right)$ conical intersection. Thus, the DSH do not necessarily follow the MEP suggested by the ab initio results.

\subsubsection{FSSH Trajectories}

This trajectory was started from the 1 ns snapshot on the $S_{2}\left(\pi, \pi^{*}\right)$ surface (figure 10.7). The trajectory stays on the $S_{2}\left(\pi, \pi^{*}\right)$ surface for more then $50 \mathrm{fs}$. It then hops directly to the ground state. The steep increase of the potential energy after the hop is most likely caused by the large amount of kinetic energy (the difference of 


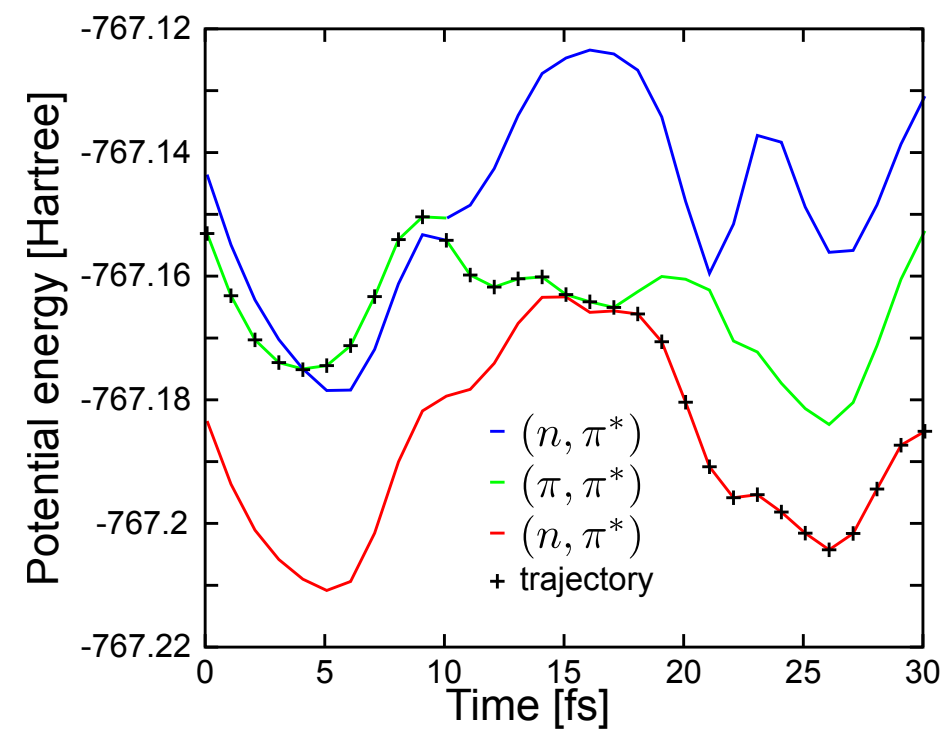

Figure 10.6: Potential energies of the three excited states of the diabatic surface hopping trajectory.

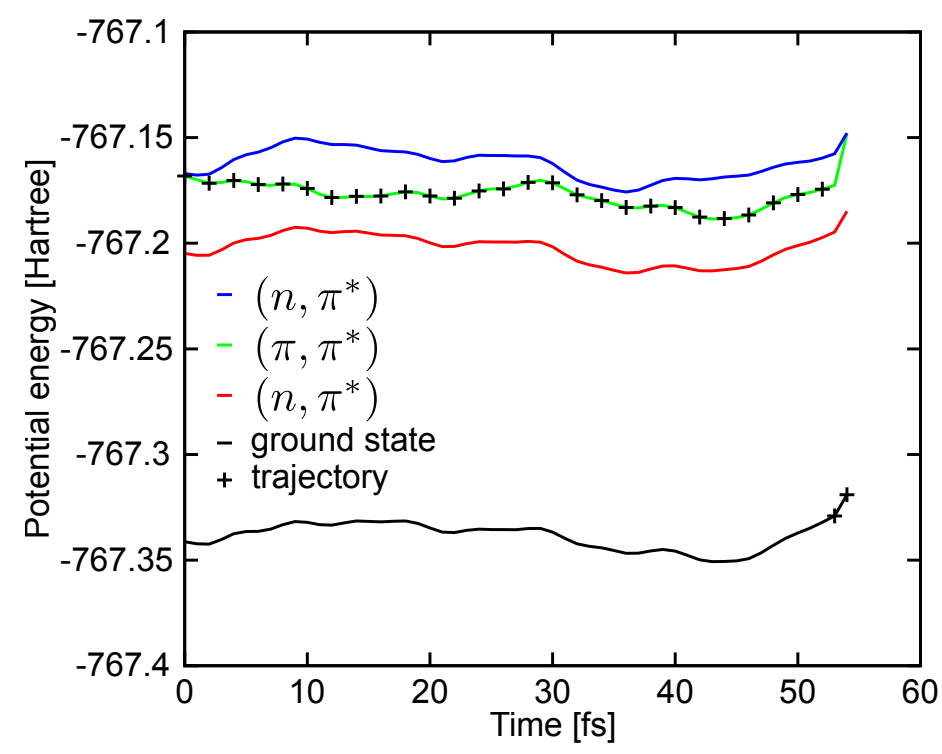

Figure 10.7: Potential energies of the three excited states of the fewest switches trajectory started from the 1 ns snapshot 
the potential energy at the hopping point, about 0.17 Hartree) put into the system during the hop. At the geometry of the $6 \mathrm{~ns}$ snaphot, the trajectory was started on the $S_{3}\left(\pi, \pi^{*}\right)$ surface. The trajectory hops immediately to the $S_{2}\left(n, \pi^{*}\right)$ surface, where it stays for $15 \mathrm{fs}$. After that, the trajectory hops to the $S_{1}\left(n, \pi^{*}\right)$ surface. Note that the $S_{3}\left(\pi, \pi^{*}\right)$ surface crosses with a doubly excited $\left(n, \pi^{*}\right)$ state after around $7 \mathrm{fs}$. The last successful trajectory was started from the $9 \mathrm{~ns}$ snapshot (figure 10.9). It was started on the $S_{3}\left(\pi, \pi^{*}\right)$ surface. After $13 \mathrm{fs}$, it hops to the $S_{2}\left(n, \pi^{*}\right)$ surface, where is stays for about $20 \mathrm{fs}$. After that it hops further to the $S_{1}\left(n, \pi^{*}\right)$ surface. After about $75 \mathrm{fs}$ the trajectory hops back to the ground state, depositing a large amount of kinetic energy into the system. Note that, again, the $\left(\pi, \pi^{*}\right)$ crosses multiple times with a doubly excited $\left(n, \pi^{*}\right)$ state.

The two FSSH trajectories (the $6 \mathrm{~ns}$ and $9 \mathrm{~ns}$ ) that reach the fluorescent $S_{1}\left(n, \pi^{*}\right)$ state do not follow the MEP suggested by the ab initio data. Both also visit the $S_{2}\left(n, \pi^{*}\right)$ state. However, the nonadiabatic molecular dynamics can again explain the excitation energy transfer from the xylene to the di-acetyl motif.

\subsection{Conclusion \& Outlook}

Both, the ab initio and the $\mathrm{QM} / \mathrm{MM}$ data, show that the excitation energy transfer from the xylene to the di-acetyl motif can be mediated by the molecular dynamics. However, the exact mechanism cannot be deduced, as the pathways suggested by the $a b$ initio data does not fit the pathways observed in the QM/MM dynamics. This discrepancy can be due to several reasons.

First, the QM/MM trajectories were run at $300 \mathrm{~K}$, whereas the ab initio data is based on single point calculations, which essentially represent the system at $0 \mathrm{~K}$. In a high dimensional system, it is highly improbable that any trajectory at room temperature will follow the MEP. Additionally, the FSSH algorithm determines the hopping probability solely from the nonadiabatic coupling between the different states (equations (8.13) and $((8.14))$. Thus, the trajectory can cross from one state to the other (e.g. from the $\left(\pi, \pi^{*}\right)$ to the $\left(n, \pi^{*}\right)$ state) even if a conical intersection with a one-dimensional seam or no conical intersection at all is encountered - as long as there is significant nonadiabatic coupling. The ab initio data shows that the $S_{2}\left(n, \pi^{*}\right) / S_{3}\left(\pi, \pi^{*}\right)$ seam is $n-1$ dimensional because the gradient difference and derivative coupling vectors are parallel (Table 10.1) and there is nonadiabatic coupling. So it is plausible that the FSSH trajectories hop from the $S_{3}\left(\pi, \pi^{*}\right)$ to the $S_{2}\left(n, \pi^{*}\right)$ state. Due to the problems in the setup and the large computational costs of the QM/MM calculations, we were only able to run a few trajectories. Therefore, 


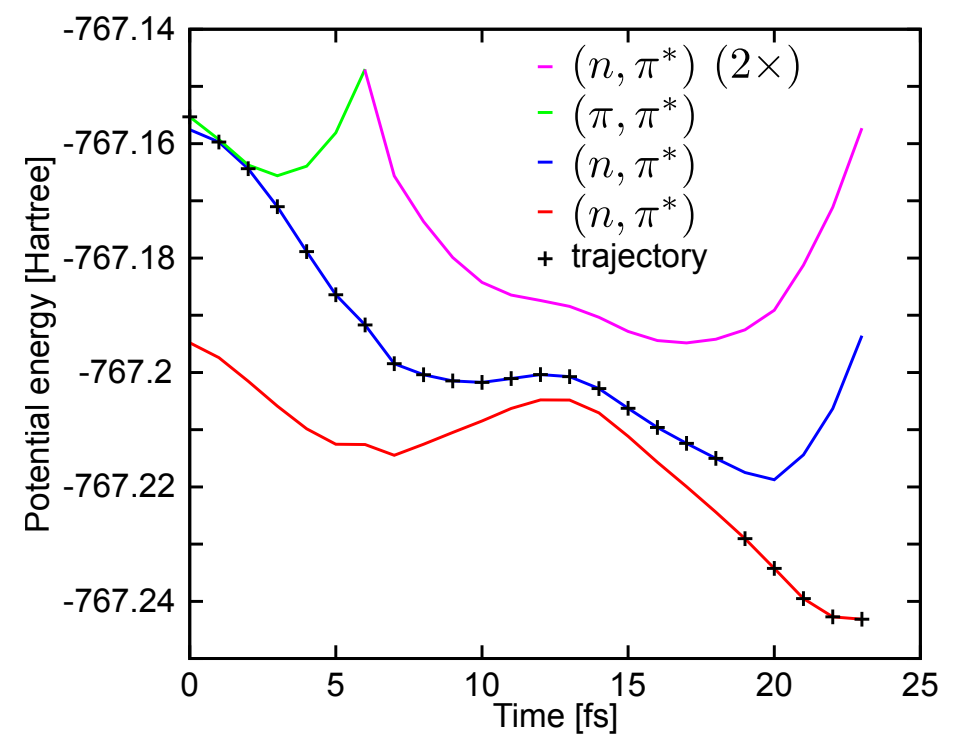

Figure 10.8: Potential energies of the three excited states of the fewest switches trajectory started from the 6 ns snapshot.

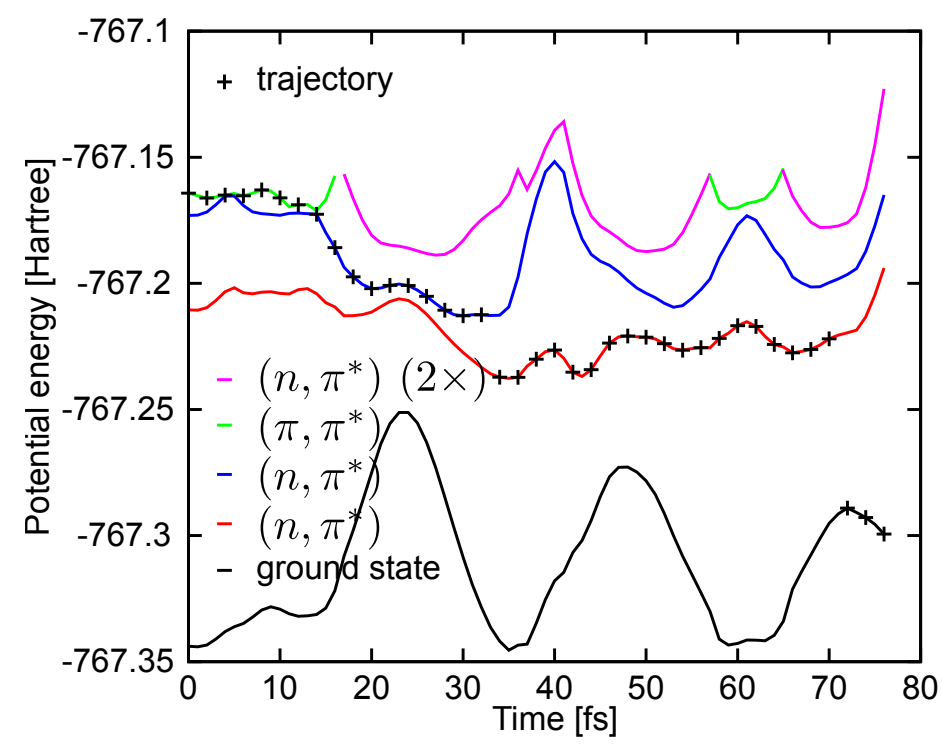

Figure 10.9: Potential energies of the three excited states of the fewest switches trajectory started from the 9 ns snapshot. 
no quantum yields can be estimated. The excitation energy transfer driven by the MD is apparently very fast ( $\leq 100 \mathrm{fs}$ ) in the $\mathrm{P}_{44}$ molecule. This timescale might be accessible to recent femtosecond laser experiments.

Irrespective of the discrepancy between the ab initio data and the QM/MM molecular dynamics, both show that the excitation energy transfer from the xylene to the di-acetyl motif can be explained in terms of the molecular dynamics on the potential energy surfaces of the different electronic states and the nonadiabatic coupling between them. This provides an alternative and a more general explanation of excitation energy transfer that does not rely on a perturbative treatment of the chromophore interaction.

A systematic study of the excited state dynamics is currently not possible for the P44 molecule due to the large computational costs of the complete active space $(\mathrm{CAS})(14,12)$ calculations. Therefore, as a possible next step, the methods used above should be applied to a computational cheaper system, i.e. a molecule the excited states of which are well described by a smaller active space. Additionally, a molecule is preferable where only two excited states are involved in the excited state dynamics for easier interpretability of the dynamics simulations. 


\section{Acknowledgements}

First of all, I am very grateful to Prof. Helmut Grubmüller for rising my interest in computational biophysics during his lecture series at the University of Göttingen and for giving me the opportunity to join his group. He always took the time for discussing my projects, providing new ideas, advise and encouraging support. He gave me the freedom and motivated me to develop and follow my own ideas.

I am also indebted to Dr. Gerrit Groenhof due to his daily supervision. He always helped me with scientific questions and technical problems in particular while working with the Gromacs source code.

I would like to thank Prof. Tim Salditt and Dr. Simone Techert, the members of my thesis committee, for finding the time for regular meetings despite their tight schedules. In addition, I am much obliged to Prof. Tim Salditt for being the second referee for my dissertation.

I thank all current and former members of the Department of Theoretical and Computational Biophysics for the great working atmosphere, fruitful discussions and the non-work related activities. Especially, I thank Eveline Heinemann for her help with all organisational issues, as well as Martin Fechner and Ansgar Esztermann for technical support.

I thank Lela Vuković for the collaboration in the PSB2 and Dr. Martial BoggioPasqua for the collaboration in the EET projects.

Financial support by International Max Planck Research School "Physics of Biological and Complex Systems" is thankfully acknowledged. 



\section{Molecular structures}

\section{A.1 Glycine}

\begin{tabular}{cccc}
\hline Atom & $x[\AA]$ & $y[\AA]$ & $z[\AA]$ \\
\hline $\mathrm{N}$ & -0.58781 & -1.82985 & 0.00000 \\
$\mathrm{C}$ & 0.48192 & -0.86468 & 0.00000 \\
$\mathrm{C}$ & 0.00000 & 0.57555 & 0.00000 \\
$\mathrm{O}$ & 1.04901 & 1.40704 & 0.00000 \\
$\mathrm{O}$ & -1.11966 & 0.96581 & 0.00000 \\
$\mathrm{H}$ & 0.72348 & 2.29818 & 0.00000 \\
$\mathrm{H}$ & 1.13065 & -0.96572 & 0.86523 \\
$\mathrm{H}$ & 1.13065 & -0.96572 & -0.86523 \\
$\mathrm{H}$ & -0.59823 & -2.40286 & -0.81480 \\
$\mathrm{H}$ & -0.59823 & -2.40286 & 0.81480 \\
\hline
\end{tabular}

Table A.1: Glycine HF/DZP equilibrium structure used for electron dynamics simulations in Section 5.3. 


\section{A.2 PENNA}

\begin{tabular}{cccc}
\hline Atom & $x[\AA]$ & $y[\AA]$ & $z[\AA]$ \\
\hline $\mathrm{C}$ & -0.01600 & -0.36200 & -0.02800 \\
$\mathrm{C}$ & 0.00500 & -0.30400 & -1.41400 \\
$\mathrm{C}$ & 1.16900 & 0.05400 & -2.08000 \\
$\mathrm{C}$ & 2.30900 & 0.35400 & -1.34700 \\
$\mathrm{C}$ & 2.28300 & 0.29400 & 0.03900 \\
$\mathrm{C}$ & 1.12200 & -0.06600 & 0.72200 \\
$\mathrm{C}$ & 1.10600 & -0.16200 & 2.23300 \\
$\mathrm{C}$ & 1.47600 & -1.56200 & 2.73200 \\
$\mathrm{~N}$ & 1.58200 & -1.65100 & 4.17300 \\
$\mathrm{C}$ & 0.31800 & -1.59200 & 4.86500 \\
$\mathrm{C}$ & 2.38600 & -2.76700 & 4.60800 \\
$\mathrm{H}$ & 3.22100 & 0.64000 & -1.85600 \\
$\mathrm{H}$ & 1.18600 & 0.10300 & -3.16100 \\
$\mathrm{H}$ & 3.17800 & 0.53600 & 0.60000 \\
$\mathrm{H}$ & 1.81500 & 0.54800 & 2.66100 \\
$\mathrm{H}$ & -0.89100 & -0.53600 & -1.97600 \\
$\mathrm{H}$ & 0.11800 & 0.12200 & 2.59500 \\
$\mathrm{H}$ & -0.93200 & -0.63800 & 0.48000 \\
$\mathrm{H}$ & 3.37800 & -2.71600 & 4.15800 \\
$\mathrm{H}$ & 2.44600 & -1.82200 & 2.30800 \\
$\mathrm{H}$ & 0.75900 & -2.29700 & 2.32900 \\
$\mathrm{H}$ & 1.94800 & -3.74400 & 4.35200 \\
$\mathrm{H}$ & 2.51300 & -2.73400 & 5.69100 \\
$\mathrm{H}$ & -0.22100 & -0.67900 & 4.61800 \\
$\mathrm{H}$ & 0.48500 & -1.58500 & 5.94300 \\
$\mathrm{H}$ & -0.33900 & -2.44600 & 4.63200 \\
\hline & & &
\end{tabular}

Table A.2: PENNA HF/6 - 31G* equilibrium structure used for electron dynamics simulations in Section 5.4. 


\section{A.3 Ab Initio Data of P44}

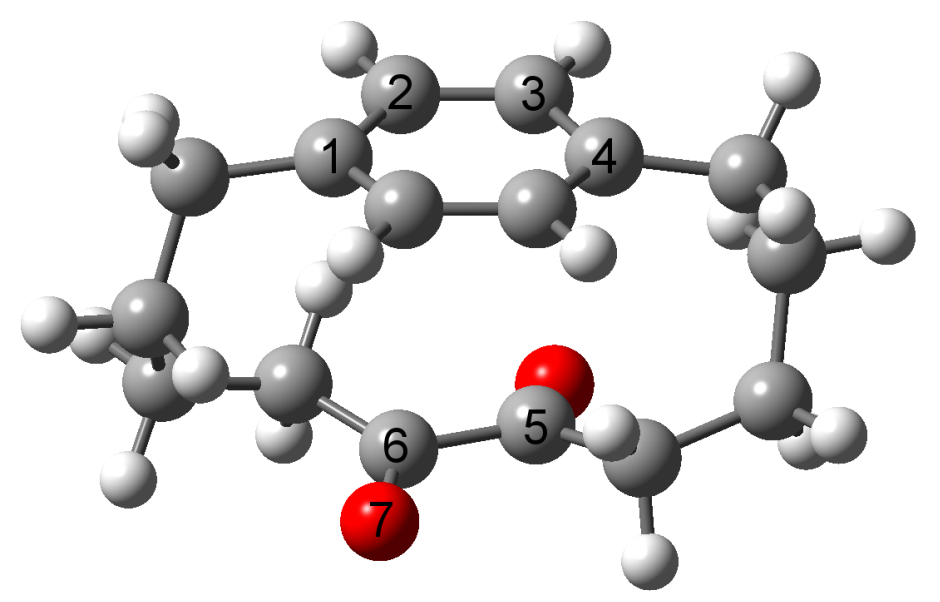

Figure A.1: CASSCF $(14,12) / 6-31 G^{*}$ optimized $C_{2}$ structure of P44.

\begin{tabular}{cccccc}
\hline & $C_{1}-C_{2}$ & $C_{2}-C_{3}$ & $C_{3}-C_{4}$ & $C_{5}-C_{6}$ & $C_{6}-O_{7}$ \\
\hline$S_{0} C_{2} \min$ & 1.399 & 1.394 & 1.401 & 1.529 & 1.208 \\
$S_{1}\left(n, \pi^{*}\right) C_{2} \min$ & 1.398 & 1.394 & 1.400 & 1.439 & 1.271 \\
$S_{2}\left(n, \pi^{*}\right) C_{2} \min$ & 1.398 & 1.394 & 1.401 & 1.387 & 1.316 \\
$S_{3}\left(\pi, \pi^{*}\right) C_{2} \min ^{*}$ & 1.437 & 1.433 & 1.438 & 1.529 & 1.208 \\
$S_{1} / S_{2} C_{2} \mathrm{CI}$ & 1.398 & 1.394 & 1.401 & 1.244 & 1.549 \\
$S_{2} / S_{3} C_{2} \mathrm{CI}$ & 1.429 & 1.425 & 1.430 & 1.497 & 1.228 \\
\hline
\end{tabular}

Table A.3: CASSCF $(14,12) / 6-31 G^{*}$ optimized $C_{2}$ structures. ${ }^{*}$ The $S_{3}\left(\pi, \pi^{*}\right) C_{2}$ minimum lies on the $S_{2}$ potential energy surface. 


\begin{tabular}{ccccc}
\hline & $S_{0}$ & $S_{1}\left(n, \pi^{*}\right)$ & $S_{2}\left(n, \pi^{*}\right)$ & $S_{3}\left(n, \pi^{*}\right)$ \\
\hline$S_{0} C_{2} \min$ & -767.399367 & -767.257960 & -767.217871 & -767.216873 \\
$S_{1}\left(n, \pi^{*}\right) C_{2} \min$ & -767.385862 & -767.270032 & -767.245312 & -767.203231 \\
$S_{2}\left(n, \pi^{*}\right) C_{2} \min$ & -767.362311 & -767.270032 & -767.245312 & -767.203231 \\
$S_{3}\left(\pi, \pi^{*}\right) C_{2} \min ^{*}$ & -767.391310 & -767.266308 & -767.251033 & -767.179816 \\
$S_{1} / S_{2} C_{2}$ CI & -767.193918 & -767.179901 & -767.179368 & -767.040529 \\
$S_{2} / S_{3} C_{2} \mathrm{CI}$ & -767.393227 & -767.259357 & -767.224818 & -767.223540 \\
\hline
\end{tabular}

Table A.4: $\operatorname{CASSCF}(14,12) / 6-31 G^{*}$ energies. ${ }^{*}$ The $S_{3}\left(\pi, \pi^{*}\right) C_{2}$ minimum lies on the $S_{2}$ potential energy surface. 


\section{Bibliography}

1. M. Beck and H.-D. Meyer. Z. Phys. D: At., Mol. Clusters, 42(2):113-129, 1997.

2. A. Ilari and C. Savino. Methods Mol. Biol., 452:63-87, 2008.

3. J. C. Kendrew, G. Bodo, H. M. Dintzis et al. Nature, 181(4610):662-666, 1958.

4. Z. Dauter. Acta Crystallogr. Sect. D, 62(1):1-11, 2006.

5. A. Geerlof, J. Brown, B. Coutard et al. Acta Crystallogr. Sect. D, 62(10):11251136, 2006.

6. M. Altarelli. Nucl. Instr. and Meth. B, 269(24):2845-2849, 2011.

7. S. Goto, T. Hatsui, A. Higashiya et al. XFEL/SPring-8 Beamline Technical Design Report Ver. 2.o (RIKEN JASRI XFEL Project Head Office, Experimental Facility Group), 2010.

8. P. Emma, R. Akre, J. Arthur et al. Nat. Photonics, 4(9):641-647, 2010.

9. K. J. Gaffney and H. N. Chapman. Science, 316(5830):1444-1448, 2007.

10. J. C. H. Spence, U. Weierstall and H. N. Chapman. Rep. Prog. Phys., 75(10):102601, 2012.

11. L. Storm and H. I. Israel. At. Data Nucl. Data Tables, 7(6):565-681, 1970.

12. M. O. Krause. J. Phys. Chem. Ref. Data, 8(2):307-327, 1979.

13. R. Neutze, R. Wouts, D. v. d. Spoel et al. Nature, 406(6797):752-757, 2000.

14. S. P. Hau-Riege, R. A. London and A. Szoke. Phys. Rev. E, 69(5):051906, 2004.

15. Z. Jurek, G. Faigel and M. Tegze. Eur. Phys. J. D, 29(2):217-229, 2004.

16. A. I. Kuleff and L. S. Cederbaum. Phys. Rev. Lett., 98(8):083201, 2007.

17. A. Dreuw and M. Head-Gordon. Chem. Rev., 105(11):4009-4037, 2005.

18. P. a. M. Dirac. Math. Proc. Cambridge Philos. Soc., 26(03):376-385, 1930.

19. C. F. Fischer. Hartree-Fock method for atoms. A numerical approach (John Wiley \& Sons, Inc.), 1977.

20. C. d. Boor. A Practical Guide to Splines, volume 27 of Applied Mathematical Sciences (Springer Verlag), $\mathbf{1 9 7 8 .}$

21. H. Bachau, E. Cormier, P. Decleva et al. Rep. Prog. Phys., 64(12):1815-1943, 2001. 
22. C. Froese Fischer, P. R. B. Ennio Arimondo and C. C. Lin. Adv. At., Mol., Opt. Phys., 55:235-291, 2008.

23. L. Cederbaum and J. Zobeley. Chem. Phys. Lett., 307(3-4):205-210, 1999.

24. J. Breidbach and L. S. Cederbaum. J. Chem. Phys., 118(9):3983-3996, 2003.

25. B. G. Levine and T. J. Martínez. Annu. Rev. Phys. Chem., 58(1):613-634, 2007. PMID: 17291184 .

26. M. A. van der Horst and K. J. Hellingwerf. Acc. Chem. Res., 37(1):13-20, 2004.

27. C. Dugave and L. Demange. Chem. Rev., 103(7):2475-2532, 2003.

28. L. Blancafort, F. Ogliaro, M. Olivucci et al. Mol. Supramol. Photochem., 13:33110, 2005.

29. K. Drukker. J. Comput. Phys., 153(2):225-272, 1999.

30. W. W. Sprenger, W. D. Hoff, J. P. Armitage et al. J. Bacteriol., 175(10):3096-3104, 1993.

31. K. J. Hellingwerf, J. Hendriks and T. Gensch. J. Phys. Chem. A, 107(8):10821094, 2003.

32. A. Xie, W. D. Hoff, A. R. Kroon et al. Biochemistry, 35(47):14671-14678, 1996.

33. U. K. Genick, G. E. O. Borgstahl, K. Ng et al. Science, 275(5305):1471-1475, 1997.

34. U. K. Genick, S. M. Soltis, P. Kuhn et al. Nature, 392(6672):206-209, 1998.

35. G. Rubinstenn, G. W. Vuister, F. A. A. Mulder et al. Nat. Struct. Biol., 5(7):568570, 1998.

36. G. Groenhof, M. Bouxin-Cademartory, B. Hess et al. J. Am. Chem. Soc., 126(13):4228-4233, 2004.

37. E. V. Gromov, I. Burghardt, H. Koeppel et al. J. Am. Chem. Soc., 129(21):67986806, 2007.

38. E. V. Gromov, I. Burghardt, J. T. Hynes et al. J. Photochem. Photobiol., A, 190(23):241-257, 2007.

39. C. M. Isborn, A. W. Goetz, M. A. Clark et al. J. Chem. Theory Comput., 8(12):5092-5106, 2012.

40. T. Förster. Ann. Phys., 437(1-2):55-75, 1948. 
41. D. L. Dexter. J. Chem. Phys., 21(5):836-850, 1953.

42. L. Stryer. Annu. Rev. Biochem., 47(1):819-846, 1978. PMID: 354506.

43. K. Truong and M. Ikura. Curr. Opin. Struct. Biol., 11(5):573-578, 2001.

44. F. Jolibois, M. J. Bearpark, S. Klein et al. J. Am. Chem. Soc., 122(24):5801-5810, 2000.

45. F. Jolibois, M. J. Bearpark and M. A. Robb. J. Phys. Chem. A, 106(17):4358-4367, 2002.

46. Y. Okuno and S. Mashiko. Int. J. Quantum Chem., 90(2):772-777, 2002.

47. Y. Okuno and S. Mashiko. Int. J. Quantum Chem., 94(1):36-43, 2003.

48. S. Hassoon, H. Lustig, M. B. Rubin et al. J. Phys. Chem., 88(25):6367-6374, 1984.

49. A. Szabo and N. S. Ostlund. Modern Quantum Chemistry (McGraw-Hill), 1989.

50. P. Löwdin. Adv. Chem. Phys, 2:207-322, 1959.

51. H. Heuser. Lehrbuch der Analysis, volume 2 (Vieweg + Teubner), 13 edition, 2008.

52. J. Broeckhove, L. Lathouwers, E. Kesteloot et al. Chem. Phys. Lett., 149(5-6):547-550, 1988.

53. A. Raab. Chem. Phys. Lett., 319(5-6):674-678, 2000.

54. M. Y. Amusia and L. Chernysheva. Computation of Atomic Processes A Handbook for the ATOM Programs (Taylor \& Francis), 1997.

55. R. Bulirsch and J. Stoer. Numerische Mathematik 2 (Springer-Verlag), 5 edition, 2005.

56. L. S. Cederbaum, W. Domcke, J. Schirmer et al. Adv. Chem. Phys., page 115-159, 2007.

57. K. D. Sevier. At. Data Nucl. Data Tables, 24(4):323-371, 1979.

58. R. S. Mulliken. J. Chem. Phys., 23(10):1833-1840, 1955.

59. H. Friedrich and H. Friedrich. Theoretical atomic physics (Springer-Verlag), 1991.

6o. E. Kálnay de Rivas. J. Comput. Phys., 10(2):202-210, 1972.

61. A. E. P. Veldman and K. Rinzema. J. Eng. Math., 26(1):119-130, 1992. 
62. J. Emsley. The elements (Clarendon press Oxford), 3 edition, 1998.

63. H. P. Kelly. Phys. Rev. A, 9(4):1582-1585, 1974.

64. A. K. Bhatia. Phys. Rev. A, 18(6):2523-2526, 1978.

65. M. Palmquist, P. L. Altick, J. Richter et al. Phys. Rev. A, 23(4):1795-1800, 1981.

66. C. A. Nicolaides, Y. Komninos and D. R. Beck. Phys. Rev. A, 27(6):3044-3052, 1983 .

67. J. A. Gaunt. Phil. Trans. Roy. Soc. London A, 228:151-196, 1929. ArticleType: research-article / Full publication date: 1929 / Copyright (C) 1929 The Royal Society.

68. A. R. Edmonds. Angular Momentum in Quantum Mechanics (Princeton University Press, Princeton, NJ), 3rd printing, with corrections, 2nd edition, 1974. Reprinted in 1996.

69. J. J. Sakurai and S. F. Tuan. Modern quantum mechanics (Benjamin/Cummings), 1985 .

70. Y. Qiu and C. F. Fischer. J. Comput. Phys., 156(2):257-271, 1999.

71. M. Galassi, J. Davies, J. Theiler et al. GNU Scientific Library Reference Manual (Network Theory Ltd.), 3 edition, 2009.

72. M. Rodbro, R. Bruch and P. Bisgaard. J. Phys. B: At. Mol. Phys., 12(15):24132447, 1979 .

73. A. I. Kuleff, J. Breidbach and L. S. Cederbaum. J. Chem. Phys., 123(4):044111044111-10, 2005.

74. S. Lünnemann, A. I. Kuleff and L. S. Cederbaum. Chem. Phys. Lett., 450(4-6):232-235, 2008.

75. J. Schirmer, A. B. Trofimov and G. Stelter. J. Chem. Phys., 109(12):4734-4744, 1998.

76. A. I. Kuleff and A. Dreuw. J. Chem. Phys., 130(3):034102-034102-8, 2009.

77. J. G. Hill. Int. J. Quantum Chem., 113(1):21-34, 2013.

78. M. J. Frisch, G. W. Trucks, H. B. Schlegel et al. Gaussian 03, Revision D.o1. 2004. Gaussian, Inc., Wallingford, CT.

79. H. Hennig, J. Breidbach and L. S. Cederbaum. J. Chem. Phys., 122(13):134104134104-9, 2005. 
8o. T. Helgaker, P. Jorgensen and J. Olsen. Molecular Electronic-Structure Theory (John Wiley \& Sons, Inc.), I edition, 2000.

81. D. Feller. J. Comput. Chem., 17(13):1571-1586, 1996.

82. K. L. Schuchardt, B. T. Didier, T. Elsethagen et al. J. Chem. Inf. Model., 47(3):1045-1052, 2007.

83. A. E. Reed and F. Weinhold. J. Chem. Phys., 78(6):4066-4073, 1983.

84. C. Peng, P. Y. Ayala, H. B. Schlegel et al. J. Comput. Chem., 17(1):49-56, 1996.

85. W. J. Hehre, R. Ditchfield and J. A. Pople. J. Chem. Phys., 56(5):2257-2261, 1972.

86. P. C. Hariharan and J. A. Pople. Theor. Chim. Acta, 28(3):213-222, 1973.

87. T. Clark, J. Chandrasekhar, G. W. Spitznagel et al. J. Comput. Chem., 4(3):294301, 1983 .

88. J. P. Perdew. Phys. Rev. B, 33(12):8822-8824, 1986.

89. A. D. Becke. Phys. Rev. A, 38(6):3098-3100, 1988.

90. T. H. Dunning and P. H. Hay. Methods of Electronic Structure Theory, volume 2 (Plenum Press), 1977.

91. C. D. Sherrill, H. F. Schaefer III and J. R. S. Per-Olov Löwdin. Adv. Quantum Chem., Volume 34:143-269, 1999.

92. J. B. Foresman, M. Head-Gordon, J. A. Pople et al. J. Phys. Chem., 96(1):135149, 1992.

93. M. Head-Gordon, R. J. Rico, M. Oumi et al. Chem. Phys. Lett., 219(1-2):21-29, 1994 .

94. M. Head-Gordon, D. Maurice and M. Oumi. Chem. Phys. Lett., 246(1-2):114121, 1995.

95. J. A. Sonk, M. Caricato and H. B. Schlegel. J. Phys. Chem. A, 115(18):4678-4690, 2011.

96. R. J. Bartlett and M. Musiał. Rev. Mod. Phys., 79(1):291-352, 2007.

97. P. V. Demekhin, A. Ehresmann and V. L. Sukhorukov. J. Chem. Phys., 134(2):024113-024113-11, 2011. 
98. L. Inhester, C. F. Burmeister, G. Groenhof et al. J. Chem. Phys., 136(14):144304144304-12, 2012.

99. E. Teller. J. Phys. Chem., 41(1):109-116, 1937.

100. G. Herzberg and H. C. Longuet-Higgins. Discuss. Faraday Soc., 35:77, 1963.

101. H. C. Longuet-Higgins. Proc. R. Soc. London, Ser. A, 344(1637):147-156, 1975.

102. M. A. Robb, F. Bernardi and M. Olivucci. Pure Appl. Chem., 67(5):783-789, 1995.

103. M. Born and R. Oppenheimer. Ann. Phys., 389(20):457-484, 1927.

104. M. Born and K. Huang. Dynamical Theory of Crystal Lattice (Oxford University Press), 1954.

105. D. R. Yarkony. Rev. Mod. Phys., 68(4):985-1013, 1996.

106. W. Kołsos. Adv. Quant. Chem., 5:99-133, 1970.

107. G. A. Worth and L. S. Cederbaum. Annu. Rev. Phys. Chem., 55(1):127-158, 2004.

108. G. Groenhof, L. V. Schäfer, M. Boggio-Pasqua et al. Excited State Dynamics in Biomolecules. In Handbook of Molecular Biophysics (Wiley-VCH Verlag GmbH \& Co. KGaA), 2009.

109. D. Marx and J. Hutter. Ab-initio Molecular Dynamics: Theory and Implementation. In Modern Methods and Algorithms of Quantum Chemistry, NIC, pages 301-449 (Forschungszentrum Jülich), i edition, 2000.

110. K. Burke. J. Chem. Phys., 136(15):150901, 2012.

111. R. O. Dror, R. M. Dirks, J. Grossman et al. Annu. Rev. Biophys., 41(1):429-452, 2012.

112. A. Warshel and M. Levitt. J. Mol. Biol., 103(2):227-249, 1976.

113. U. C. Singh and P. A. Kollman. J. Comput. Chem., 7(6):718-730, 1986.

114. M. J. Field, P. A. Bash and M. Karplus. J. Comput. Chem., 11(6):700-733, 1990.

115. B. O. Roos, P. R. Taylor and P. E. Siegbahn. Chem. Phys., 48(2):157-173, 1980.

116. D. R. Hartree, W. Hartree and B. Swirles. Phil. Trans. Roy. Soc. London A, 238(790):229-247, 1939.

117. R. Shepard. Adv. Chem. Phys., page 63-200, 2007. 
118. B. H. Lengsfield, P. Saxe and D. R. Yarkony. J. Chem. Phys., 81(10):4549-4553, 1984 .

119. J. C. Tully. J. Chem. Phys., 93(2):1061-1071, 1990.

120. G. Granucci and M. Persico. J. Chem. Phys., 126(13):134114-134114-11, 2007.

121. R. A. de Groot and J. Nadrchal, editors. Gromacs - a parallel computer for molecular-dynamics simulations, volume 92 of Physics Computing (World Scientific, Singapore), 1993.

122. H. Berendsen, D. van der Spoel and R. van Drunen. Comput. Phys. Commun., 91(1-3):43-56, 1995.

123. E. Lindahl, B. Hess and D. van der Spoel. J. Mol. Model., 7(8):306-317, 2001.

124. D. Van Der Spoel, E. Lindahl, B. Hess et al. J. Comput. Chem., 26(16):1701-1718, 2005.

125. B. Hess, C. Kutzner, D. van der Spoel et al. J. Chem. Theory Comput., 4(3):435447, 2008.

126. P. J. Knowles and N. C. Handy. Chem. Phys. Lett., 111:315-321, 1984.

127. P. J. Knowles and H.-J. Werner. Chem. Phys. Lett., 115(3):259-267, 1985.

128. H.-J. Werner and P. J. Knowles. J. Chem. Phys., 82:5053, 1985.

129. P. J. Knowles and H.-J. Werner. Chem. Phys. Lett., 145:514-522, 1988.

130. H.-J. Werner and P. J. Knowles. J. Chem. Phys., 89:5803-5814, 1988.

131. P. J. Knowles and N. C. Handy. Comput. Phys. Commun., 54:75-83, 1989.

132. R. D. Amos, J. S. Andrews, N. C. Handy et al. Chem. Phys. Lett., 185:256-264, 1991.

133. P. J. Knowles, J. S. Andrews, R. D. Amos et al. Chem. Phys. Lett., 186(2-3):130136, 1991.

134. P. J. Knowles and H.-J. Werner. Theor. Chim. Acta, 84:95-103, 1992.

135. P. J. Knowles, C. Hampel and H.-J. Werner. J. Chem. Phys., 99:5219-5227, 1993.

136. R. Hockney, S. Goel and J. Eastwood. J. Comput. Phys., 14(2):148-158, 1974.

137. H. J. C. Berendsen, J. P. M. Postma, W. F. van Gunsteren et al. J. Chem. Phys., 81(8):3684-3690, 1984.

138. G. Bussi, D. Donadio and M. Parrinello. J. Chem. Phys., 126(1):014101, 2007. 
139. W. F. van Gunsteren and H. J. Berendsen. Angew. Chem. Int. Ed., 29(9):992-1023, 2003.

140. W. F. van Gunsteren, S. R. Billeter, A. A. Eising et al. Biomolecular Simulation: The GROMOS96 Manual and User Guide (Vdf Hochschulverlag, Zürich), 1996.

141. L. D. Schuler and W. F. Van Gunsteren. Mol. Sim., 25(5):301-319, 2000.

142. Y. Duan, C. Wu, S. Chowdhury et al. J. Comput. Chem., 24(16):1999-2012, 2003.

143. F. Jensen. Introduction to computational chemistry (John Wiley \& Sons, Inc.), 2007.

144. J. Hinze. J. Chem. Phys., 59(12):6424, 1973.

145. H.-J. Werner. J. Chem. Phys., 74(10):5802, 1981.

146. E. Fabiano, G. Groenhof and W. Thiel. Chem. Phys., 351(1-3):111-116, 2008.

147. J. C. Tully. Int. J. Quantum Chem., 40(25):299-309, 1991.

148. E. Fabiano, T. Keal and W. Thiel. Chem. Phys., 349(1-3):334-347, 2008.

149. C. Zhu, A. W. Jasper and D. G. Truhlar. J. Chem. Theory Comput., I(4):527-540, 2005.

150. K. Palczewski. Annu. Rev. Biochem., 75(1):743-767, 2006.

151. L. A. Gunaydin, O. Yizhar, A. Berndt et al. Nat. Neurosci., 13(3):387-392, 2010.

152. W. F. Van Gunsteren and H. J. C. Berendsen. Mol. Sim., 1(3):173-185, 1988.

153. L. Vukovic, C. F. Burmeister, P. Kral et al. Photoisomerization Control Mechanism in Protonated Schiff Bases, 2012. Unpublished.

154. M. W. Mahoney and W. L. Jorgensen. J. Chem. Phys., 112(20):8910, 2000.

155. A. W. Jasper, S. N. Stechmann and D. G. Truhlar. J. Chem. Phys., 116(13):5424, 2002.

156. M. Ben-Nun, J. Quenneville and T. J. Martínez. J. Phys. Chem. A, 104(22):5161$5175,2000$.

157. A. D. Becke. J. Chem. Phys., 98:5648, 1993.

158. J. Kóna, P. Zahradník and W. M. Fabian. J. Org. Chem., 66(15):4998-5007, 2001.

159. C. Lee, W. Yang and R. G. Parr. Phys. Rev. B, 37(2):785, 1988.

16o. S. H. Vosko, L. Wilk and M. Nusair. Can. J. Phys., 58(8):1200-1211, 1980. 


\section{Lebenslauf von Carl Friedrich Burmeister}

\section{Persönliche Daten}

Name

Anschrift

Nationalität

\section{Ausbildung}

1990-2003

2003

2004-2009

2009

2009-2013
Carl Friedrich Burmeister

Schlegelweg 4

37075 Göttingen

deutsch

Geb. am 26.11.1983 in Braunschweig

Grundschule \& Orientierungstufe \& Gymnasium

(Wilhelm-Gymnasium, Braunschweig)

Erwerb der allgemeinen Hochschulreife

Studium der Physik an der Georg-August-Universität Göttingen

Erwerb des Diploms in Physik

Promotionsstudium der Physik an der Georg-August-Universität Göttingen in der Arbeitsgruppe von Prof. Helmut Grubmüller am Max-Planck Institut für Biophysikalische Chemie 